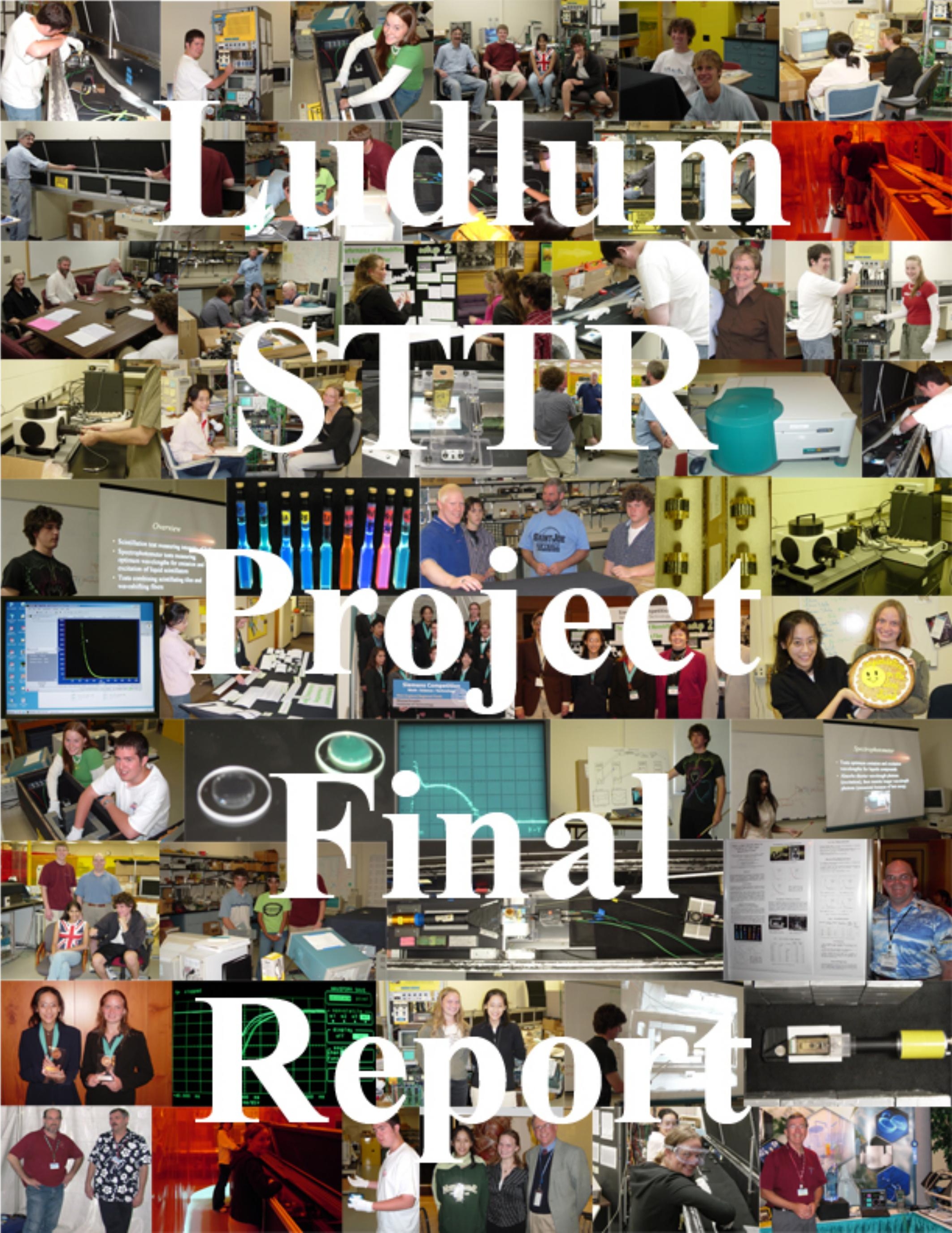




\title{
Waveshifters and Scintillators for lonizing Radiation Detection
}

December 11, 2007

\section{Subcontract associated with the U.S. Department of Energy SBIR Program Phase II Grant \# DE-FG02-03ER86171 to Ludlum Measurements, Inc.}

\author{
B. Baumbaugh, J. Bishop, D. Karmgard, J. Marchant, M. McKenna, R. Ruchti and M. Vigneault \\ Department of Physics and QuarkNet Center \\ University of Notre Dame, Notre Dame, Indiana 46556-5670
}

\author{
L. Hernandez and C. Hurlbut \\ Ludlum Measurements, Inc. \\ Sweetwater, Texas 79556-3209
}

\begin{abstract}
We have been developing scintillation and waveshifter materials for the detection of ionizing radiation under an STTR program with Ludlum Measurements, Inc. During this program, we have developed several new waveshifter materials, which are comparable in efficiency and faster in fluorescence decay than the standard material Y11 (K27) used in particle physics for several decades now. Additionally, new scintillations materials useful for fiber tracking have been developed which have been compared to $3 \mathrm{HF}$. Lastly, we have been developing liquid scintillators and paint-on scintillators and waveshifters for high radiation environments.
\end{abstract}

\section{Table of Contents}

$\begin{array}{lll}\text { Section } & \text { Topic } & \text { Page Number }\end{array}$

$1 \quad$ Tasks under this Subcontract 2

2 Introduction 3

3 Characterization of Solid and Liquid Scintillators and Waveshifters 4

$4 \quad$ Scintillating Tile with Wavelength-Shifting Fiber 8

$5 \quad$ Scintillating Fiber Studies for Attenuation Length \& Efficiency 28

$6 \quad$ Liquid and Paint-on Scintillators and Waveshifters 32

$7 \quad$ Comments on Educational and Workforce Development 38

$8 \quad$ Conclusions 39

$9 \quad$ Appendices (publications) $\quad 40$ 


\section{Section 1. Tasks under this Subcontract}

1. Characterization of scintillator and waveshifter materials for emission, absorption and excitation, fluorescence efficiency, and decay time.

a. Status: complete.

b. Reporting: see Sections 3-6.

c. Comment: This was a critical task to identify and/or produce and test new dyes for scintillation and waveshifting.

2. Characterization of scintillating tiles for efficiency and uniformity.

a. Status: complete.

b. Reporting: see Section 4.

c. Comment: This is a critical task that is directly related to Task 4 below.

3. Characterization of waveshifter fibers of various diameters, lengths and dye concentrations.

a. Status: made a comparative study only of fibers of $940 \mu \mathrm{m}$ diameter, $1 \mathrm{~m}$ length, and standard concentrations.

b. Reporting: see Section 4 .

c. Comment: Future work could be carried out in this area - on dye concentration and fiber length. This task is a follow-on to effort under Tasks 2 and 4.

4. Characterization of scintillating tiles with waveshifter fiber readout.

a. Status: complete.

b. Reporting: see Section 4.

c. Comment: This task is critical for Tile/Fiber calorimetry in a variety of experiments.

5. Characterization of scintillating fibers in various diameters for attenuation length and detected photoelectron yield. Comparisons will be made with $3 \mathrm{HF}$ scintillating fibers of similar diameters and lengths.

a. Status: made a comparative study only of fibers of $835 \mu \mathrm{m}$ diameter and $3 \mathrm{~m}$ length.

b. Reporting: see Section 5 .

c. Comment: future work with varying dye concentrations and diameters would be useful. Follow-up studies under this task could provide useful tracking materials for a variety of experiments.

6. Characterization of tiles and fibers before and after irradiation.

a. Status: This task was not implemented.

b. Comment: It was determined that there were more immediately urgent issues to consider, for example alternative materials such as liquids.

c. Replacement Task 6. This task was replaced with an extensive study of liquids and paint-on scintillator and waveshifter materials.

d. Status: Liquids studied extensively for fluorescence characteristics and efficiency. Paint-on material studies started, but work is just beginning. The liquids are important for collider, fixed-target and neutrino experiments. Paintons may find use as shifters on the face of silicon photomultipliers: SiPM, of potential interest for LHC and ILC applications.

e. Reporting: see Section 6 .

7. Participation in the writing of the final report. 


\section{Section 2: Introduction}

This program is focused on the development of new scintillation and waveshifter materials and structures for the detection of ionizing radiation. Applications may be found in high energy physics, nuclear physics, nuclear medicine, homeland security and allied fields.

The program has concentrated on tile/fiber, fiber, and bulk materials - solids, liquids, and paint-on materials - including their fluorescence emission and absorption, decay time, efficiency, and attenuation characteristics.

The motivation behind the R\&D program is several-fold. First, conventional materials used for scintillation and waveshifting applications were developed several decades ago. Y11 (K27) used as a waveshifter is bright, but has a relatively slow fluorescence decay time $(\tau>7 \mathrm{nsec})$. When used to read out conventional scintillators, an overall decay time of order $\tau \sim 17 \mathrm{nsec}$ is observed. This is right at the margin at LHC where the crossing interval (time between beam collisions) is $25 \mathrm{nsec}$. In applications where faster timing is essential, materials with more rapid fluorescence response would be desirable, if not essential. Our goal in this program was to develop and study materials which would afford significant reduction in response time, whilst maintaining fluorescence efficiency at a level comparable to or better than Y11.

For scintillating fiber tracking, the standard used for several decades now is double-clad polystyrene fiber of sub-millimeter diameter and length of several meters containing the fluorescent dyes pTP (para-Terphenyl) and 3HF (3-hydroxyflavone). The response time of this material is acceptable $(\tau \sim 7 \mathrm{nsec})$ and the efficiency is very good when used with VLPC (Visible Light Photon Counter) readout. However the 3HF is photosensitive with rapid deterioration of the optical behavior of the material under exposure to long wavelength UV and visible light. To use it requires special handling under yellow or longer wave lighting, and light tighting of any structures that intend to utilize the material over long periods of time (the usual case for most experimental applications). Hence, it is desirable to consider alternatives that provide the excellent performance of $3 \mathrm{HF}$, yet avoid the undesirable characteristics of this material. Several materials have been tested and compared to $3 \mathrm{HF}$.

In many modern experiments, detectors are exposed to significant radiation fields and to harsh environments. A goal of this project was to explore detection media that would be suitable for operation under such conditions. Alternatives to plastic scintillators that were explored in this program include liquid scintillators and waveshifters and paint-on materials. Several candidates were identified that hold promise for further study. 


\section{Section 3: Characterization of Solid and Liquid Scintillators and Waveshifters}

\section{A. Waveshifters:}

In this program we studied the spectral properties and responses to ionizing radiation of solid and liquid materials. Initially we focused on the production or identification and characterization of promising dyes that were potentially soluble in organic plastic (polystyrene and polyvinyltoluene base) and/or in liquids (pseudocumene and PXE). A key element was to match the dye materials for optimal coupling and performance. A standard scintillator with which we made relative comparisons is EJ204, whose emission spectrum is shown in Figure 1.

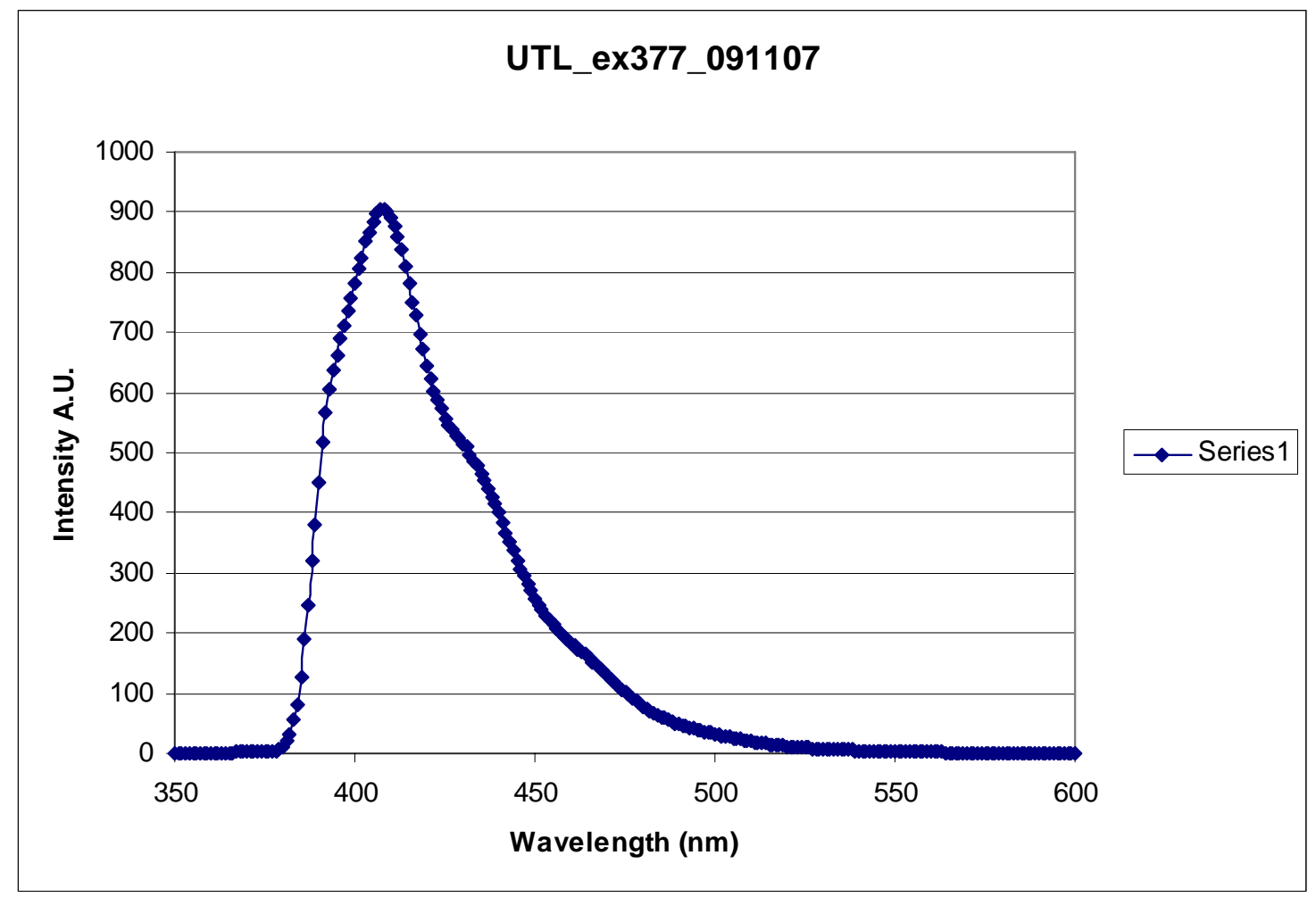

Figure 1. Emission spectrum of EJ204 scintillator. Maximum is near $409 \mathrm{~nm}$.

For efficient waveshifting, the excitation of the waveshifter should have strong spectral overlap with and high extinction coefficient in the region of the EJ204 emission. And the shifter material should have sufficient Stokes' Shift so that, at low concentration, the light produced by the shifter can propagate over significant distance, with low probability of self-absorption.

A number of dyes were developed for this program: DSB1, DSB2, DSF1, DSF2, BDOC, DTPO, AH238B and OX-550F. These were incorporated into solid or liquid hosts and compared to fluorescent standards such as Y11 and BBQ. Spectra and decay 
characteristics for several of these materials are presented in the figures and tables below. Figure 2 shows the emission and excitation spectra of EJ204, Y11 (K27), DSB1, and DSB2. These spectra were measured with a Hitachi 2000 Spectrophotometer. This data indicates that several of these new materials are spectrally interchangeable with Y11. Figure 3 shows spectra for several other shifters DSF1, DSF2, and AH238B in plastic or organic liquid base. These were measured with a Varian Cary Eclipse Fluorescence Spectrophotometer attached to a custom data acquisition system.

Note that of these, AH238B is the longest wavelength (yellow-red) emitter.
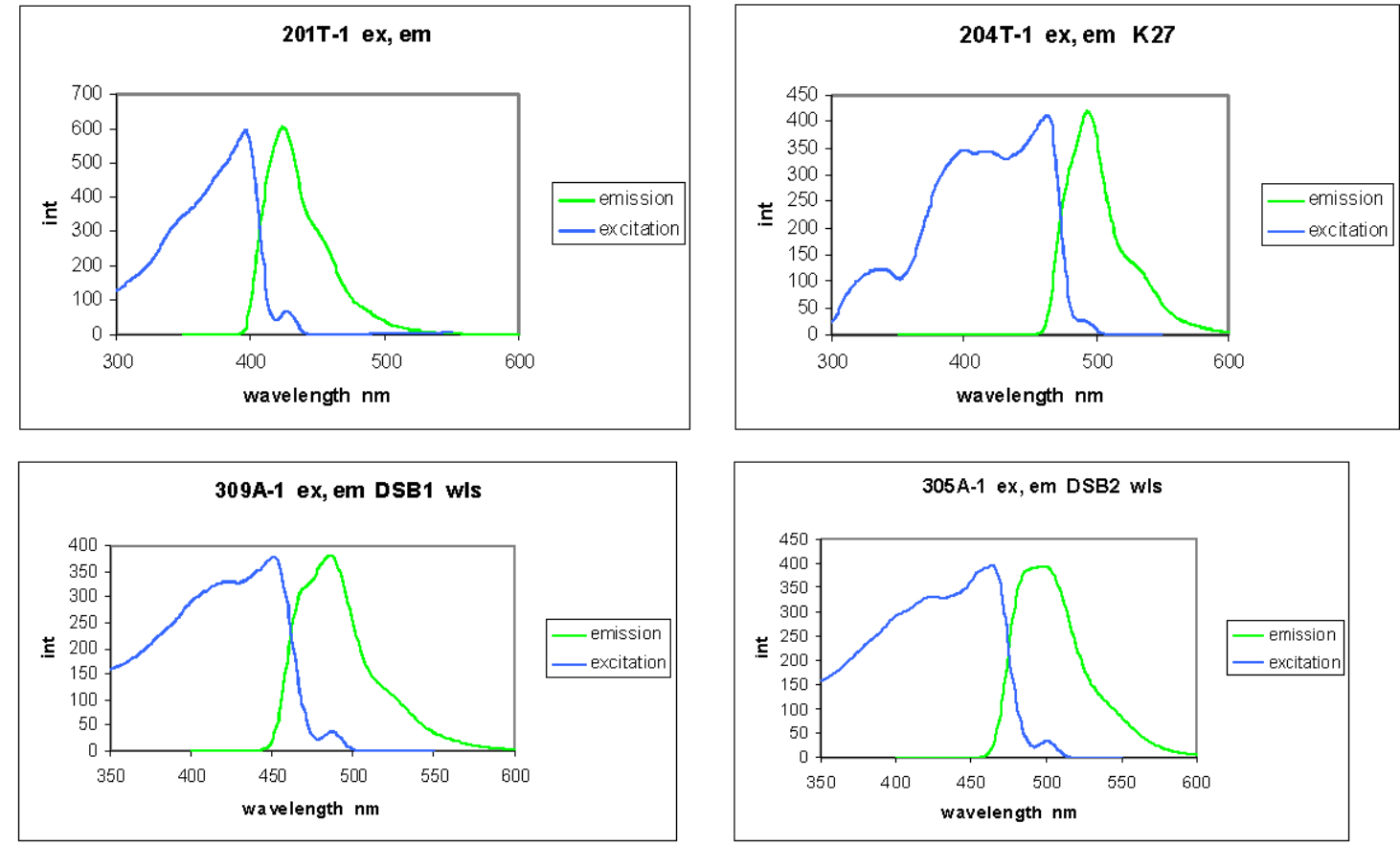

Figure 2. Emission and absorption spectra in plastic polyvinyltoluene base. Standard blue-violet emitting scintillator EJ204 (upper left). Standard Y11 waveshifter (upper right). New DSB1 waveshifter (lower left). New DSB2 waveshifter (lower right). The spectral excitation and emission properties of DSB1 and DSB2 are seen to be generally similar to Y11. 


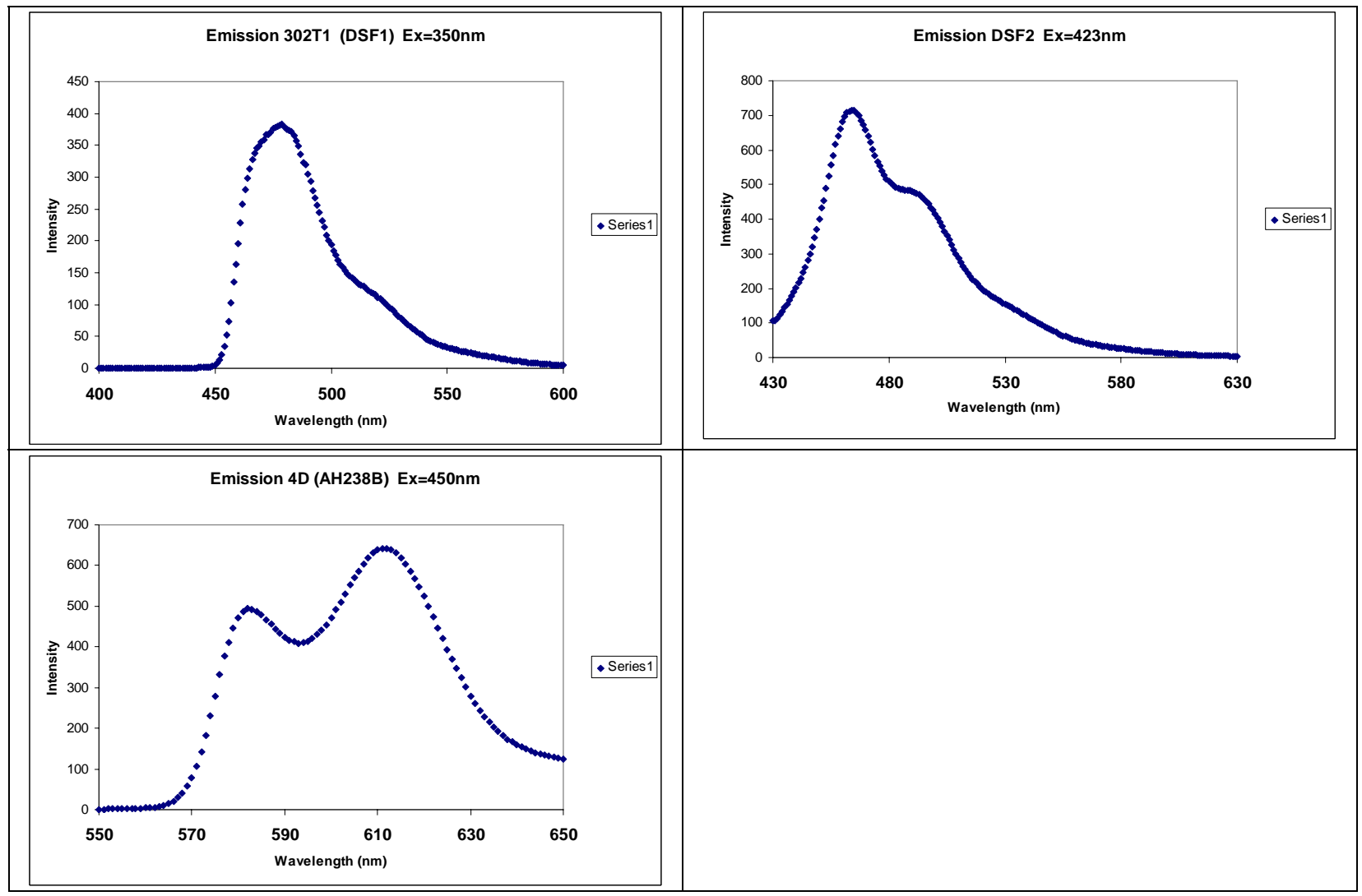

Figure 3. Emission spectra for several waveshifters. DSF1 waveshifter organic liquid base (upper left). DSF2 waveshifter in PVT base (upper right), AH238B waveshifter in organic liquid base (lower left).

The fluorescence decay times of several materials are presented in Figure 4. These were measured with a Horiba Jobin Yvon Pulsed Diode Laser Excitation System with TBX-04 Picosecond Photo Detection Module. Samples were excited at $\lambda=377 \mathrm{~nm}$ or $\lambda=457 \mathrm{~nm}$ and the fluorescence decay observed at emission maximum. As can be seen in Figure 4, the photo-excited fluorescence decay time of DSF1 $(\tau=1.3 \mathrm{~ns})$ is significantly faster than standard Y11 $(\tau=7.4 \mathrm{~ns})$ by a factor of over 5. The decay of AH238B is found to be slower $(\tau=11.5 \mathrm{~ns})$ but, as can be seen in Figure 3, the emission wavelength for this dye is considerably longer (in the yellow/red).

These measurements indicate that we have suitable material for incorporation into plastic fiber materials and for liquid scintillator applications. 


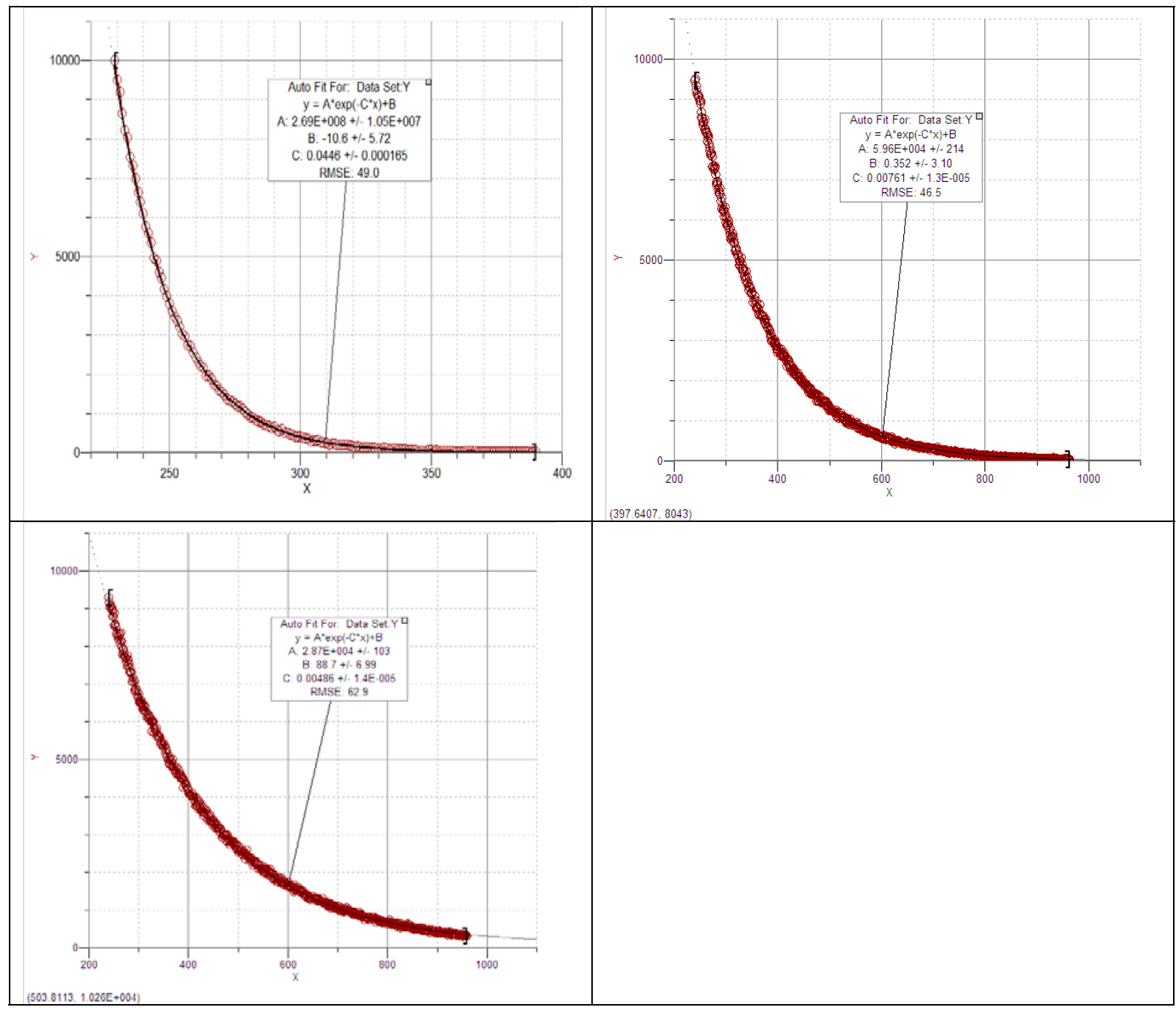

Figure 4. Fluorescence decay times excited by a Diode Laser at $377 \mathrm{~nm}$. Light is detected at the emission maximum for the samples: DSF1 in organic liquid (1.3 nsec, upper left); Y11 in PVT (7.4 nsec, upper right); AH238B in organic liquid (11.5 nsec, lower left). 


\section{Section 4: Scintillating Tile with Wavelength-Shifting Fiber}

\section{A. Details of Apparatus and Measurement}

Tile/fiber measurements are relevant for a number of high energy physics experiments. For example they find use in collider experiments, neutrino experiments, and others. The detector (sensor) consists of a volume of scintillator (either a scintillating tile or strip) and an embedded waveshifter fiber of double-clad construction. For the tests reported here, scintillation tile fabricated by Ludlum Measurements, Inc. and designated Eljen Technologies EJ204 was used to convert the energy of charged particles (either from radioactive sources or from cosmic rays) to light in the blue-violet part of the visible spectrum. The emission spectrum for this material (peak near 409nm) is shown above in Figure 1, and the detection and testing apparatus is shown in Figure 5 with schematic information in Figure 6.

Ball groove channels were machined into the EJ204 tiles to support the position of the waveshifter fiber. The geometry of the tile was $11 \mathrm{~cm} \mathrm{x} 11 \mathrm{~cm}$ area and $1 / 4$ " thickness. The waveshifter fiber was of double-clad material, of $940 \mu \mathrm{m}$ diameter and $1 \mathrm{~m}$ length and fabricated by Kuraray Corp. The waveshifter fiber was inserted into the ball groove. Prior to its insertion into the tile, the insertion-end of the fiber was aluminized to provide reflection back into the fiber of any light produced within the fiber. The non-aluminized end was presented to the photocathode of a photomultiplier tube, either a Hamamatsu R943-02 GaAs PMT or a Hamamatsu H7422P-40 GaAsP PMT. Figure 7 shows the spectral responsivity (and the quantum efficiency) of these two PMTs from the Hamamatsu online catalogue. The tubes provide improved sensitivity to longer wavelength visible light over conventional multialkali PMTs - important for detection of light from the waveshifters developed in this project.

The tiles were wrapped in Tyvek ${ }^{\circledR}$, a diffuse reflector, to contain the scintillation light produced from radioactive sources or cosmic rays within the tile as much as possible. A telescope of trigger counters was placed above and below the tile to detect the presence of penetrating electrons (from a beta source ${ }^{106} \mathrm{Ru}$ or ${ }^{90} \mathrm{Sr}$ with energies of a few MeV) or from cosmic rays (generally muons of energies $\leq 1 \mathrm{GeV}$ ). 


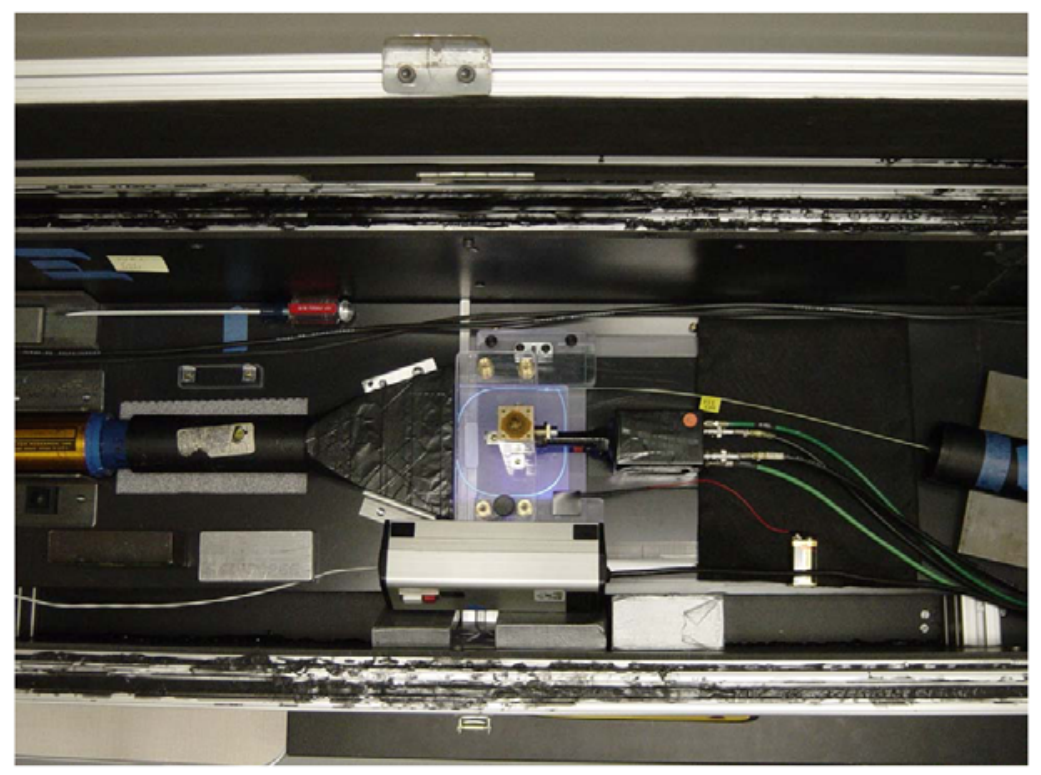

Figure 5. Detection system for tile-fiber measurements in which fluorescence efficiency of fiber waveshifters is studied. Scintillating tile with embedded wavelength-shifter fiber is visible in the center of the figure. The tile has been unwrapped for purposes of this image. Normally it would be wrapped in Tyvek ${ }^{\circledR}$, which acts as a diffuse reflector to contain the scintillation light within the tile. A radioactive source is placed at the center of the tile for scintillation testing. To illuminate the tile for this picture, a black light was used to excite the tile material and the shifter. The tile glows blue-violet in the picture. The waveshifter fiber appears whitish-blue in the image and is arranged in a "sigma" format. The fiber then extends to a PMT at the right-hand side, either a GaAs or GaAsp PMT, capable of detecting longer wavelength visible light with good efficiency.
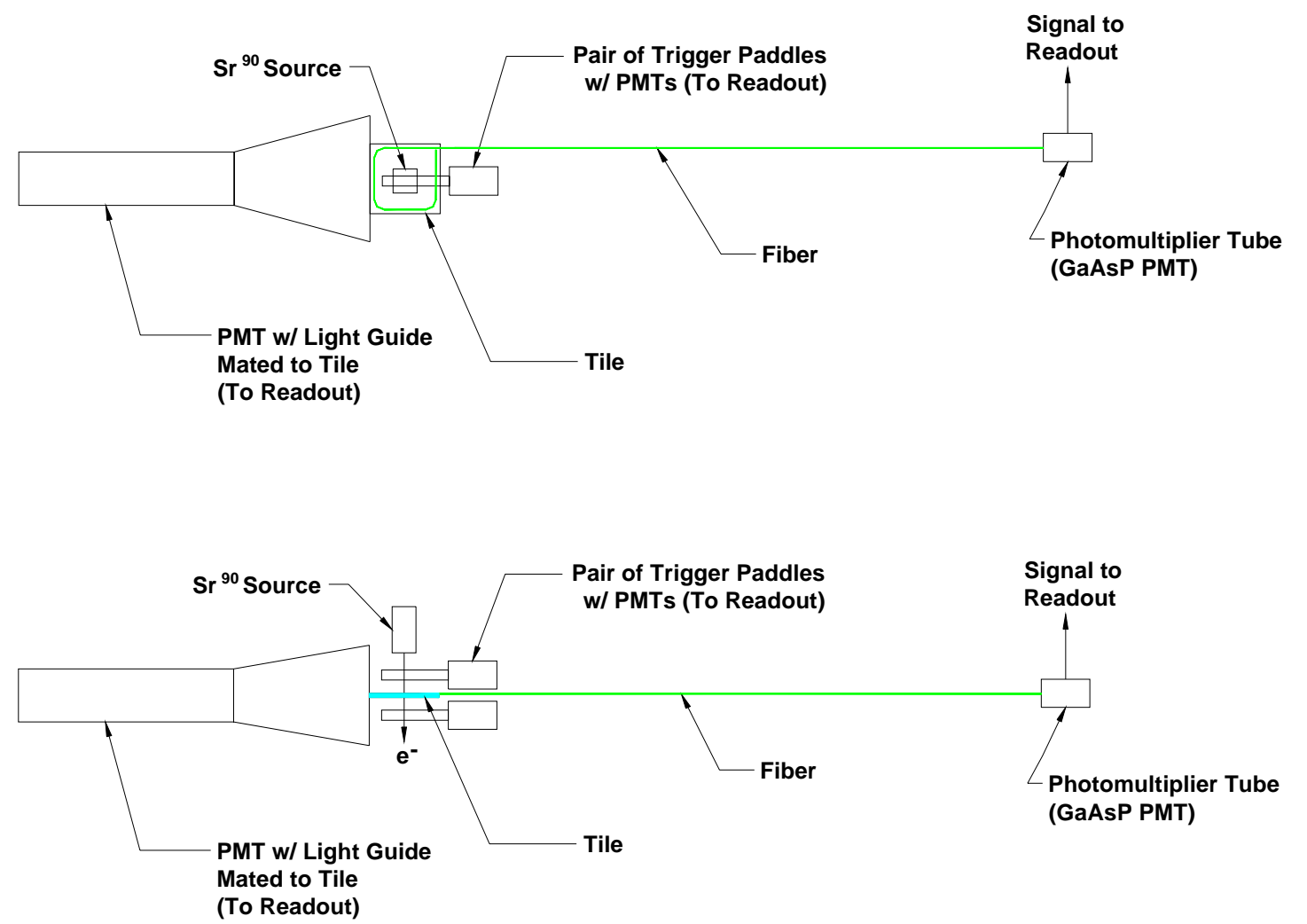

Figure 6. Schematic of the detection system for tile-fiber measurements in which fluorescence efficiency of fiber waveshifters is studied: top view (upper); side view (lower); compare this to the actual setup shown in Figure 5. 
The purpose of the tests was to measure the relative performance of different waveshifter fibers, and to compare this performance relative to Y11 waveshifting fiber. The Y11 is the standard material used in the high energy physics community (for CDF, ATLAS and CMS calorimetric applications).
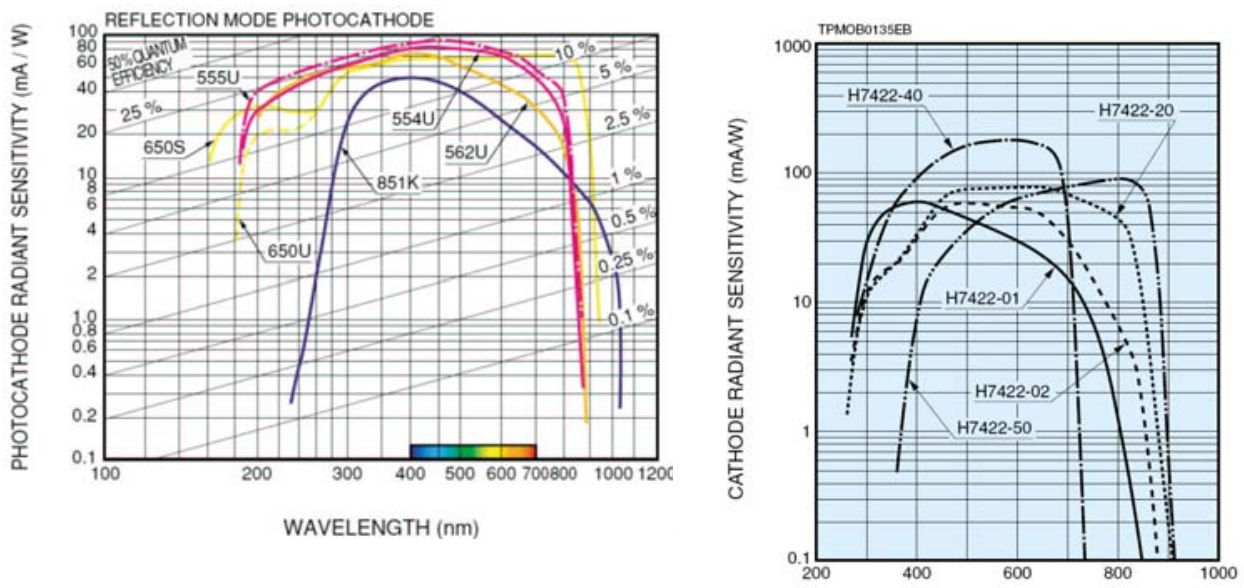

Figure 7. Spectral responsivity and quantum efficiency of various photocathodes of PMTs made by Hamamatsu. Left: for the R943 GaAs PMT the curve of relevance is 650S. Right: for the GaAsP PMT the curve of relevance is H7422P-40. At $500 \mathrm{~nm}$ wavelength, the quantum efficiencies are approximately $15 \%(\mathrm{GaAs})$ and $42 \%(\mathrm{GaAsP})$. At $600 \mathrm{~nm}: 9 \%(\mathrm{GaAs})$ and $37 \%(\mathrm{GaAsP})$

The detection system shown above in Figures 5 and 6 includes a three-fold coincidence of trigger counters, including a PMT that views the scintillation tile itself and two other thin independent counters, one above and one below the tile. The coincidence of these counters was used as a gate for the signal from the waveshifter fiber, embedded in a groove in the tile. The optical signals from the fiber were transduced by the PMT into electronic pulses; 2048 of such pulses were averaged, displayed and recorded on an HP54502A digitizing oscilloscope and read into a computer. Such signals were recorded for triggers that occurred in response to ${ }^{90} \mathrm{Sr}$ or ${ }^{106} \mathrm{Ru}$ beta sources or cosmic rays.

Figure 8 displays the recorded signals from the tile/fiber combinations. As can be seen, the best performance is observed for DSB1 shifter and it is superior to Y11 in both overall signal and in signal response time. DSF1 and DSF2 have lower overall signal, but have even faster response. The data show that for DSF1, the full width at half maximum (FWHM) is 9.0ns, and a fit to the tail with a single exponential function reveals a decay time of $\tau=6.1 \mathrm{~ns}$. DSB1 response is similar to DSF1, whereas the comparable values of these quantities for Y11 are FWHM $=17 \mathrm{~ns}$ and $\tau=12.1 \mathrm{~ns}$ respectively, a factor of nearly 2 times slower behavior in each category. Table I summarizes the results of these measurements and others. 

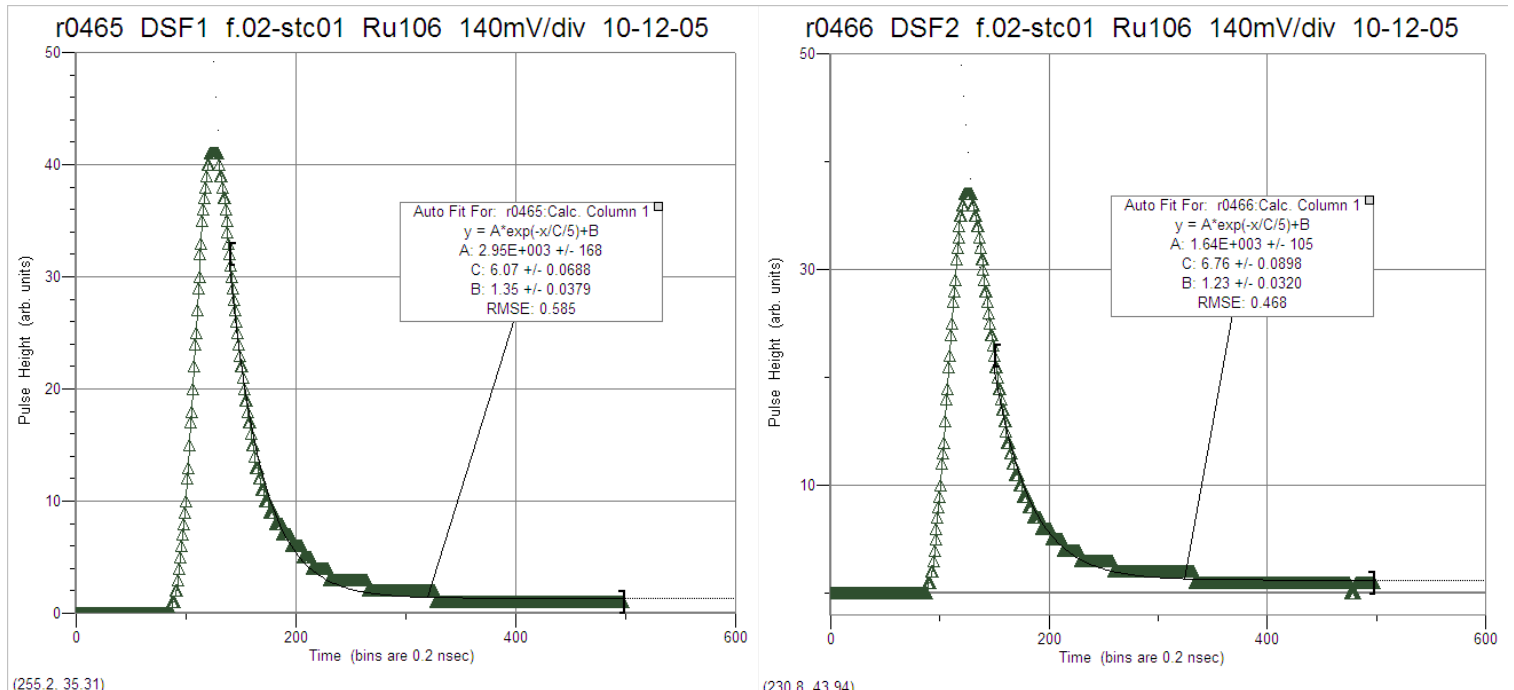

$(255.2,35.31)$

(230.8. 43.94)

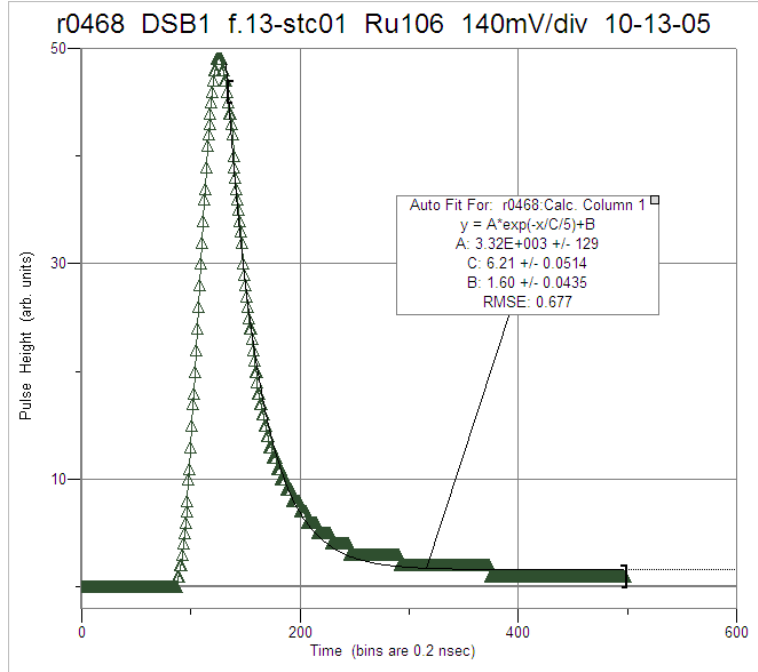

$(235.0,37.90)$

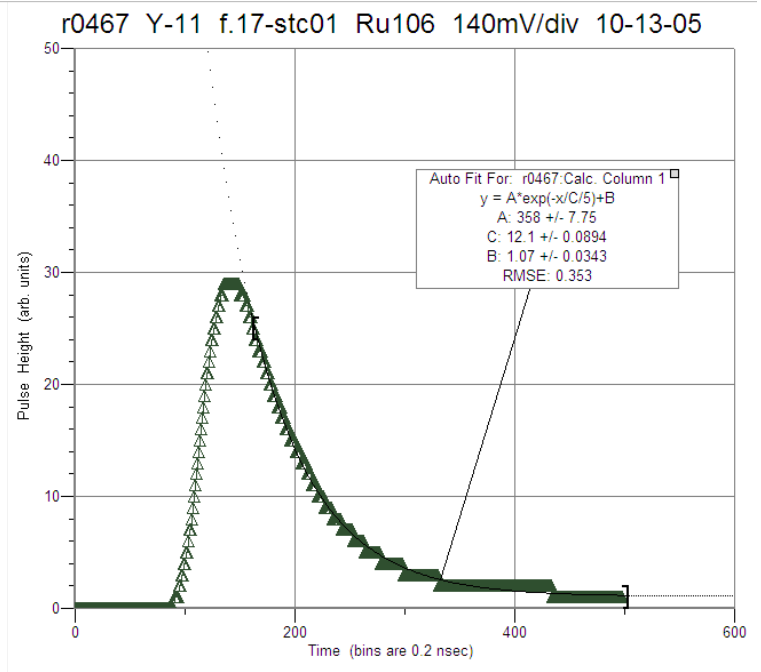

$(164.6,41.73)$

Figure 8. Averaged output pulses from various waveshifters in double-clad fiber of $940 \mu \mathrm{m}$ diameter and $1 \mathrm{~m}$ length reading out EJ204 scintillating tile of $6 \mathrm{~mm}$ thickness and $11 \mathrm{~cm} \times 11 \mathrm{~cm}$ area. Photosensor is a GaAsP PMT. DSF1 (upper-left); DSF2 (upper-right); DSB1 (lower-left); Y11 in PVT (lower-right).

TABLE I

SUMMARY OF WAVESHIFTING EFFICIENCY FOR SAMPLE MATERIALS

$\begin{array}{lllll}\text { Waveshifter } & \begin{array}{l}\text { Emission wavelength } \\ \text { Peak value }\end{array} & \begin{array}{l}\text { Efficiency rel to } \\ \text { Y11 }\end{array} & \begin{array}{l}\text { Decay Time } \\ \text { Source Exc. }\end{array} & \begin{array}{l}\text { FWHM Source } \\ \text { Exc. }\end{array} \\ & & & \begin{array}{l}\text { Decay Time } \\ \text { Laser Exc. }\end{array} \\ \text { Y11 (K27) } & 492 \mathrm{~nm} & 1 & 12.1 \mathrm{~ns} & 7.4 \mathrm{~ns} \\ \text { DSB1 } & 489 \mathrm{~nm} & 1.02 & 6.2 \mathrm{~ns} & 9.4 \mathrm{~ns} \\ \text { DSF1 } & 480 \mathrm{~nm} & 0.84 & 6.1 \mathrm{~ns} & 9.0 \mathrm{~ns} \\ \text { DSF2 } & 480 \mathrm{~nm} & 0.79 & 6.6 \mathrm{~ns} & 9.6 \mathrm{~ns}\end{array}$


During these tile fiber tests a phenomenon of "multiple pulsing" was observed (see Figure 9). Questions emerged as to the origin of the delayed pulses. One hypothesis was that the pulses were due to after-pulsing in the PMTs; another was that the scintillation light was scattered within the tile due to the Tyvek ${ }^{\circledR}$ wrapping; another was that it was due to the excitation of high order modes (off-axis) in the waveshifting fibers. A series of studies were undertaken in which the waveshifter fiber was placed in different geometric orientations relative to the scintillation tile. These variations are shown in Figure 10.

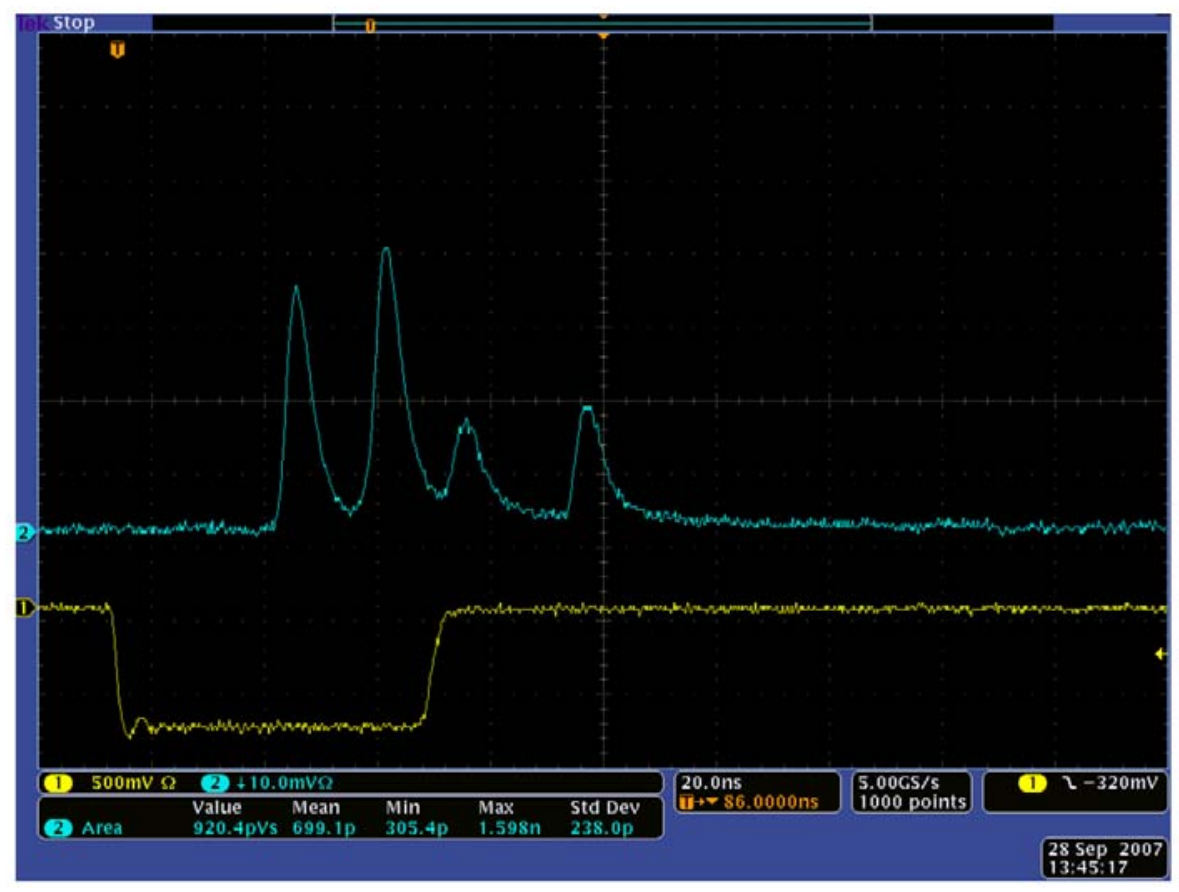

\section{DPO4104 - 1:41:35 PM 9/28/2007}

Figure 9. Scope trace (in blue) of an individual tile fiber signal as recorded in the GaAsP PMT and displayed on a Tektronix digital oscilloscope. As can be seen, a single triggered electron passing through the tile fiber system often produces "multiple pulses" in the PMT (in this case, 4 pulses). The number of such pulses depends upon the geometry of the waveshifter fiber placement relative to the scintillating tile. 


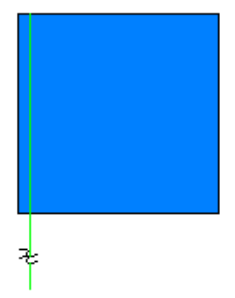

Fiber on Top of Tile (Offset) - A

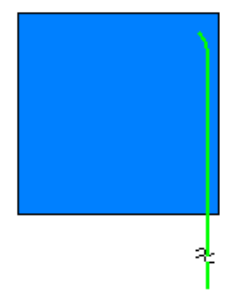

Fiber Inserted Into $1 / 4$ of Tile Groove - E

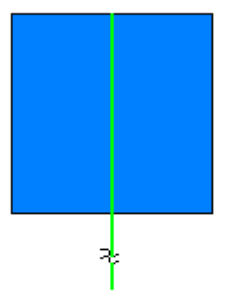

Fiber on Top of Tile (Centered) - B

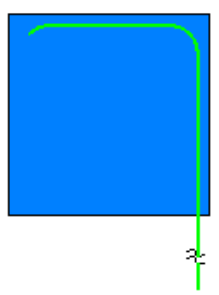

Fiber Inserted Into $1 / 2$ of Tile Groove - F

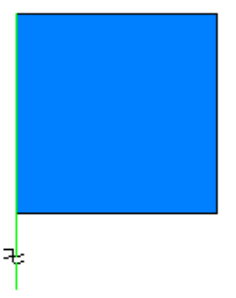

Fiber on Side of Tile - C

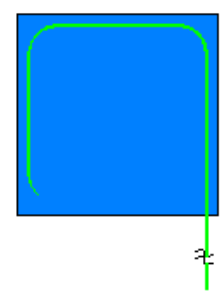

Fiber Inserted Into $3 / 4$ of Tile Groove - G

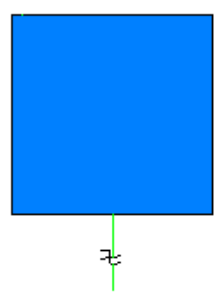

Fiber End on Edge of Tile - D

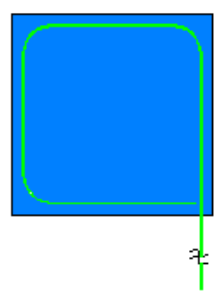

Fiber Inserted Fully Into Tile Groove - $\mathrm{H}$

Figure 10. Geometric arrangements of waveshifter fiber relative to a scintillating tile during tests. Configurations are labeled A through H. The "sigma" configuration in the lower right-hand corner is the standard form used by CDF and CMS and is the H configuration.

The measurements of averaged signal shapes recorded with a Tektronix DPO 4104

Digital Phosphor Oscilloscope are presented in Figures 11-34. Studies included the fiber orientation, as indicated in Figure 10, the waveshifter type (DSB1, DSF1,Y11), and the form of the cover material surrounding the tile: Tyvek ${ }^{\circledR}$, Tedlar ${ }^{\circledR}$ or black felt.

By varying the orientation of the fiber away from side illumination, the number of multipulses was reduced, suggesting that illumination through the sidewalls of the shifter fiber leads to the generation of off-axis modes and, hence, multiple pulses. It was also observed that the envelope of these pulses (the pulse average) is reduced in the faster waveshifter materials. The end-on geometry (Configuration D in Figure 10) affords the least possibility of multiple pulses, but at the expense of relatively poor and ineffectual optical coupling/waveshifting.

These studies indicate that the response time of certain calorimetry might be controlled by fiber orientation and cladding materials. Additionally, the fibers used for these studies were of $1 \mathrm{~m}$ length. For longer fibers, the probability of propagating the off-axis modes is less likely, as the optical path for such modes is longer than for modes that are more closely meridianal - and because there is a non-unity reflection coefficient at boundaries. 


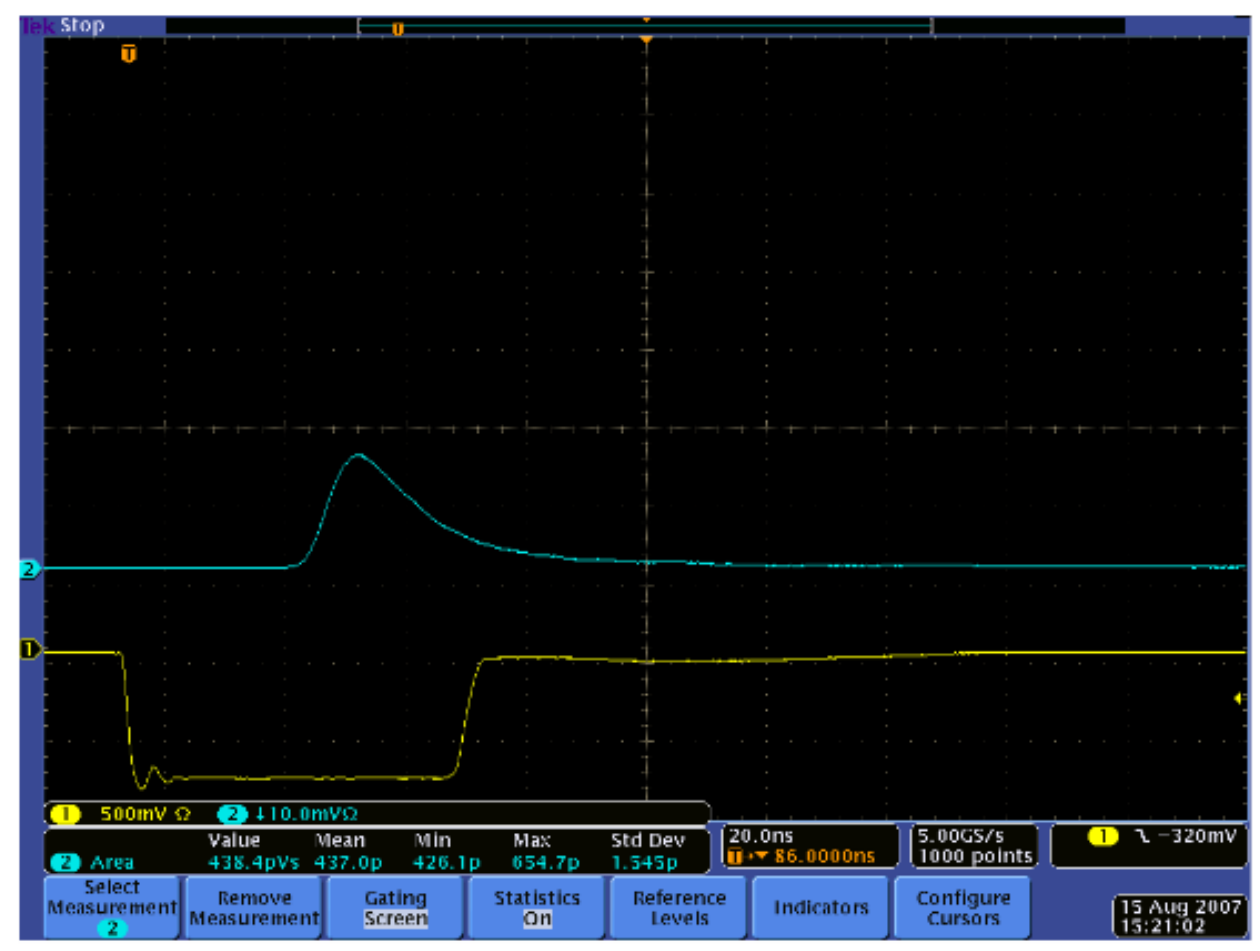

\section{r1333, Y11,f.17 on edge stc03, 8-15-07}

Figure 11. Tile/fiber configuration A: the pulse-averaged signal for configuration (blue scope trace). Five hundred and twelve pulses were averaged. The type was EJ204 wrapped in Tyvek ${ }^{\circledR}$. Shifter fiber is Y11.

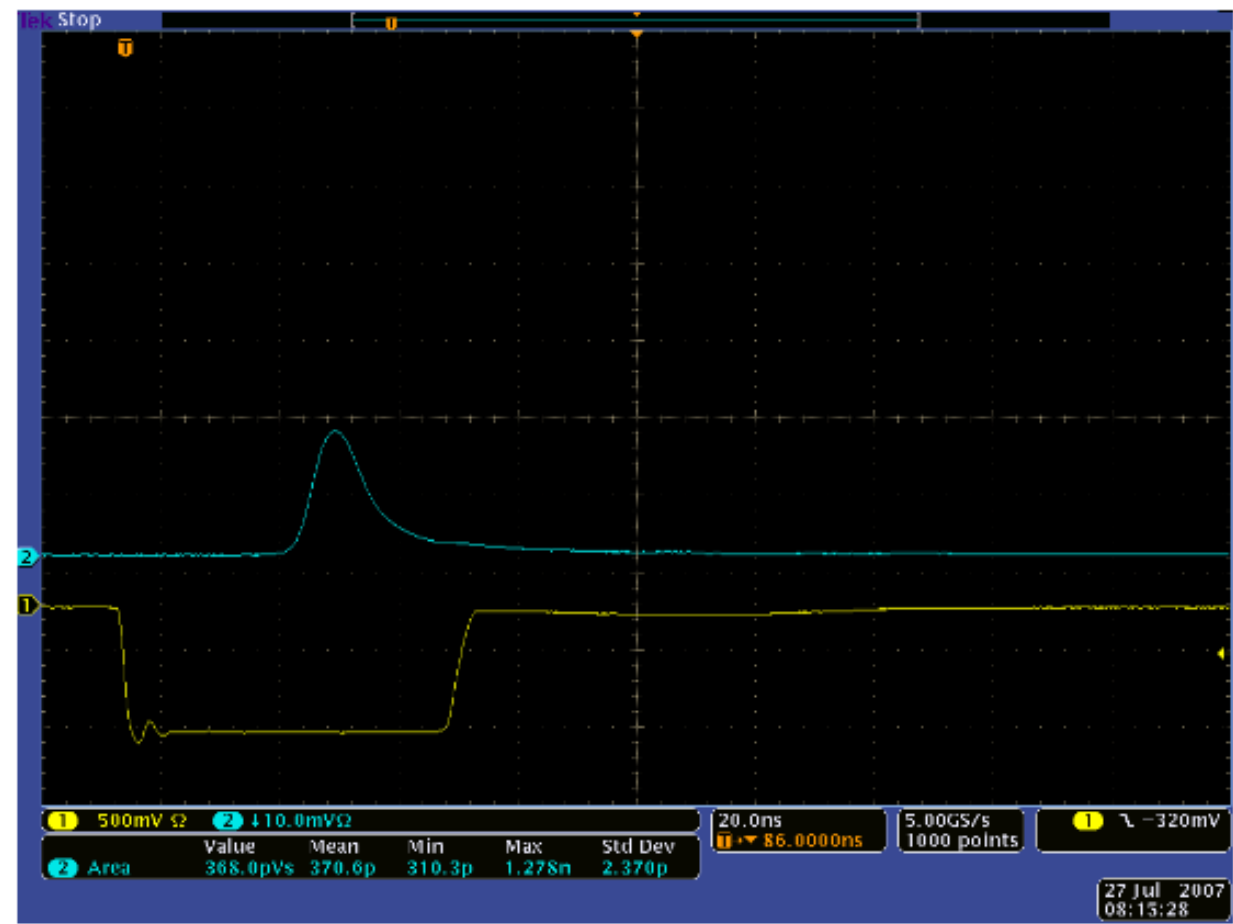

\section{DPO4104 - 8:16:47 AM 7/27/2007}

Figure 12. Tile/fiber configuration A: the pulse-averaged signal for configuration (blue scope trace). Five hundred and twelve pulses were averaged. The type was EJ204 wrapped in Tyvek ${ }^{\circledR}$. Shifter fiber is DSB1. 


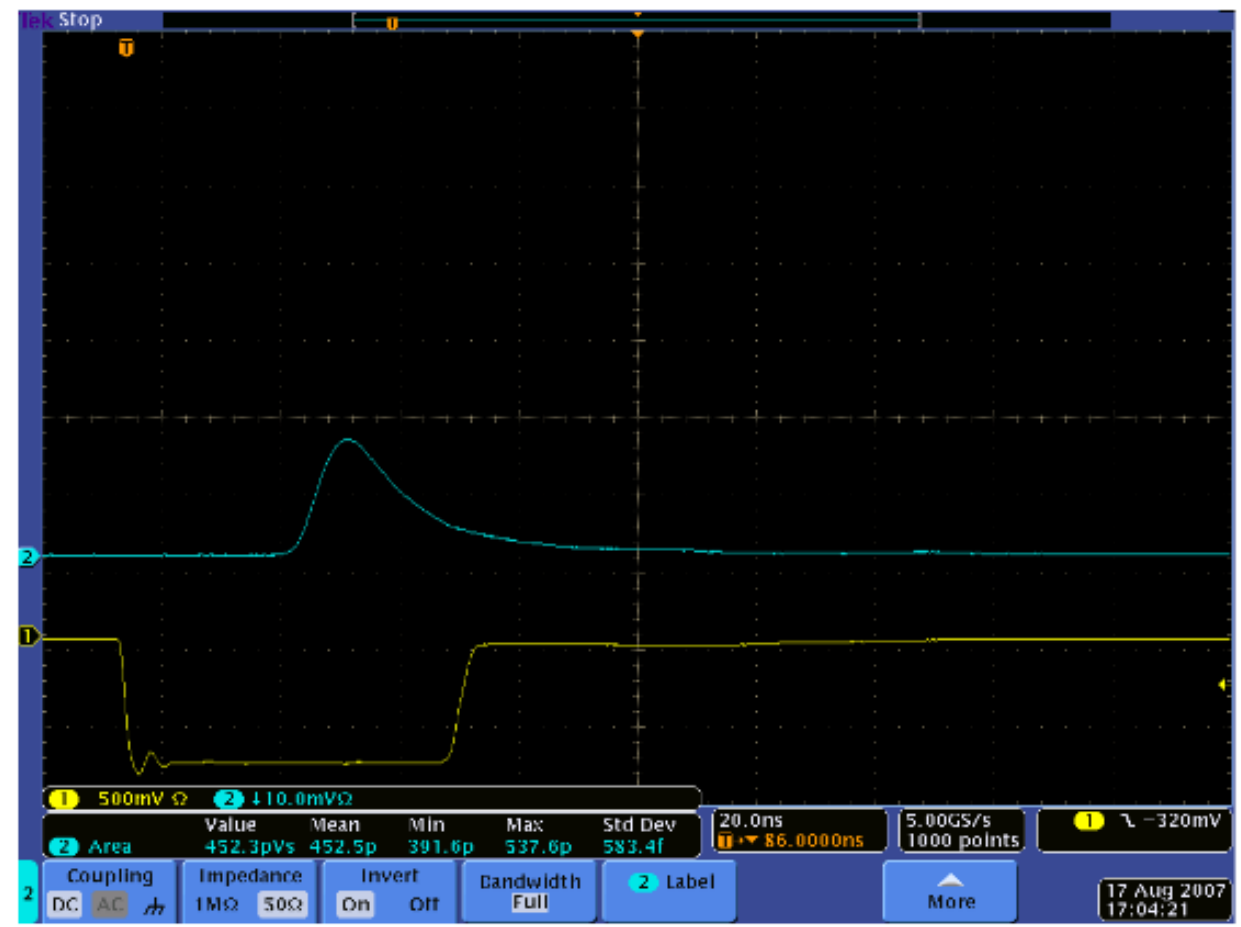

r1335, Y11,f.17 top mid stc03, 8-17-07

Figure 13. Tile/fiber configuration B: the pulse-averaged signal for configuration (blue scope trace). Five hundred and twelve pulses were averaged. The type was EJ204 wrapped in Tyvek ${ }^{\circledR}$. Shifter fiber is Y11.

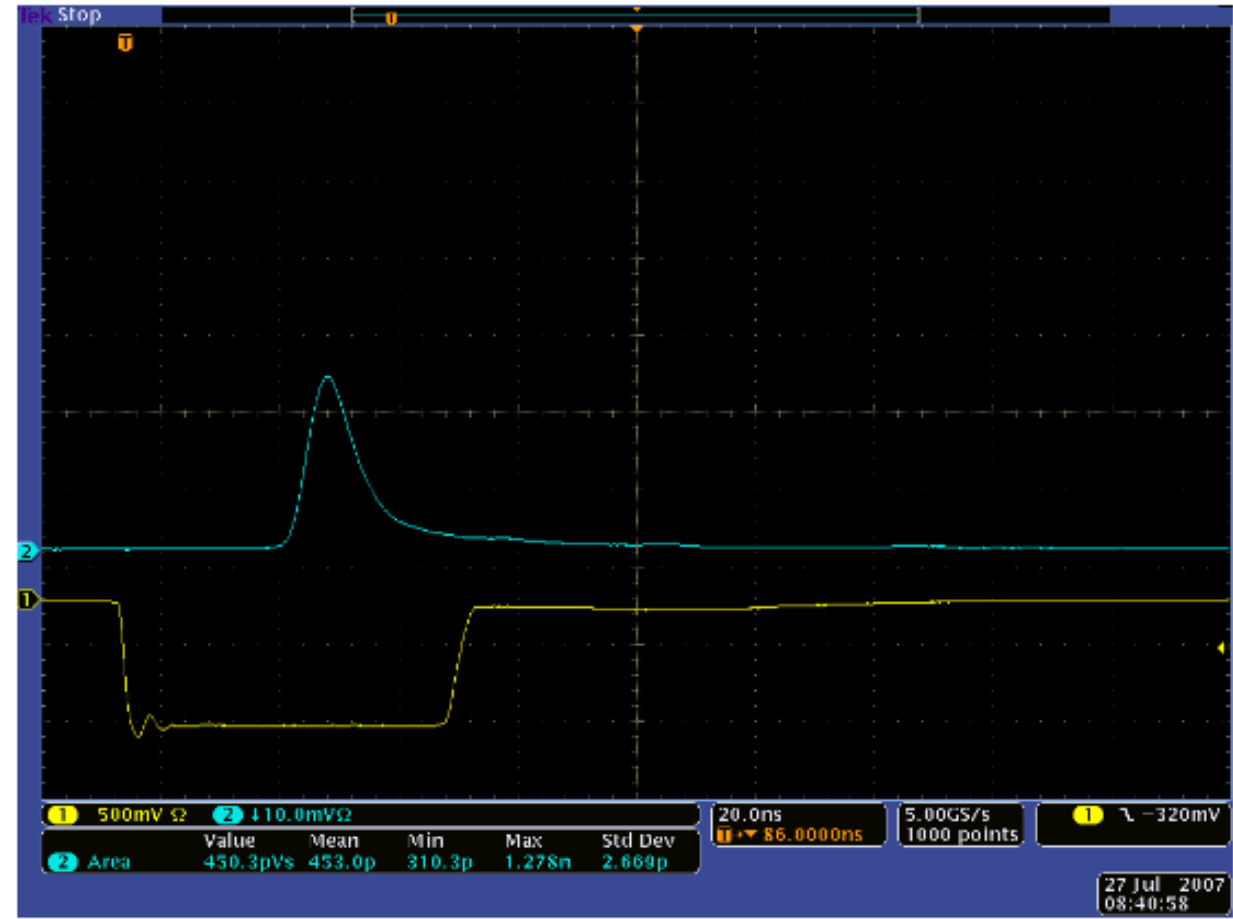

\section{DPO4104 - 8:42:19 AM 7/27/2007}

Figure 14. Tile/fiber configuration B: the pulse-averaged signal for configuration (blue scope trace). Five hundred and twelve pulses were averaged. The type was EJ204 wrapped in Tyvek ${ }^{\circledR}$. Shifter fiber is DSB1. 


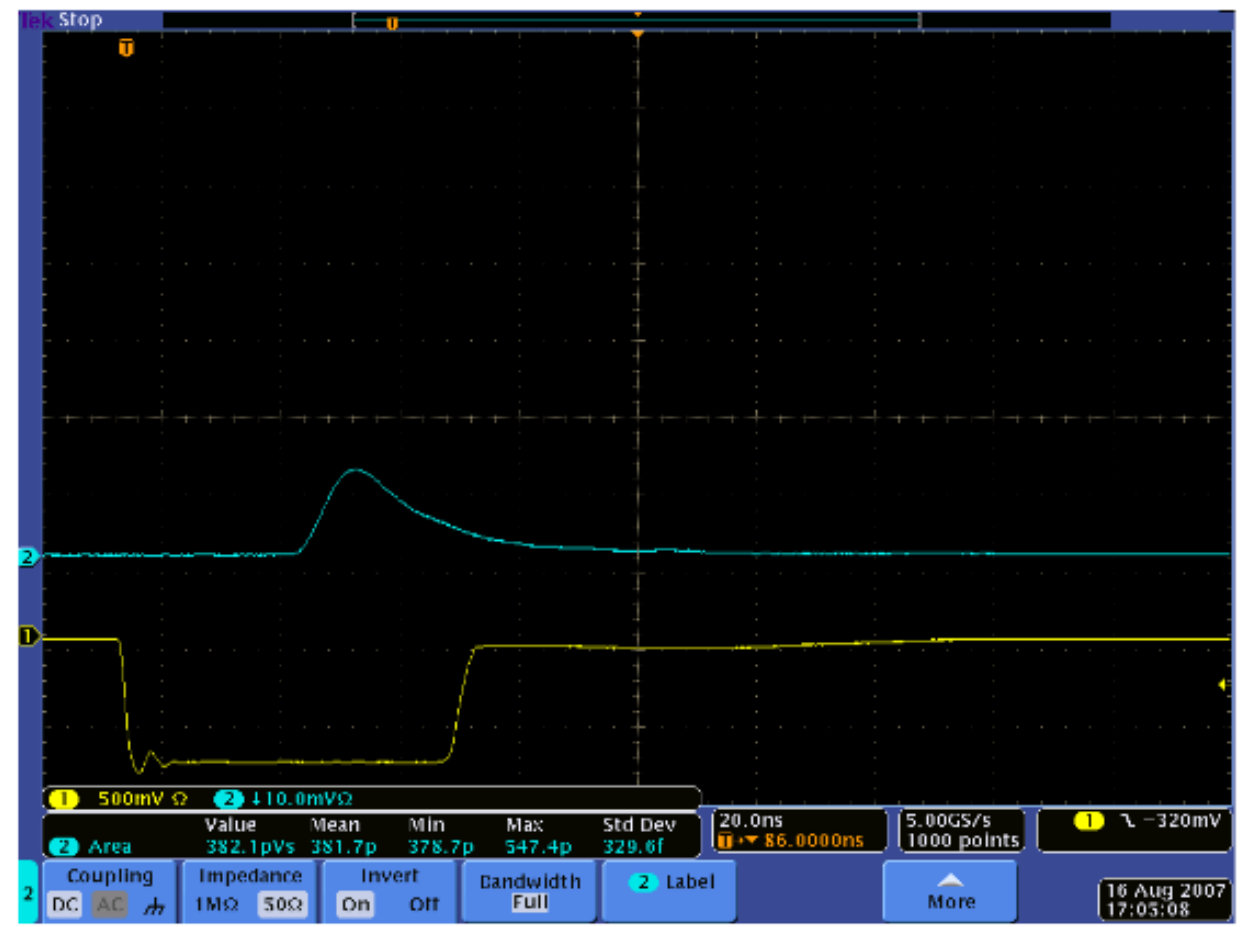

\section{r1334, Y11,f.17 top side stc03,8-16-07}

Figure 15. Tile/fiber configuration C: the pulse-averaged signal for configuration (blue scope trace). Five hundred and twelve pulses were averaged. The type was EJ204 wrapped in Tyvek ${ }^{\circledR}$. Shifter fiber is Y11.

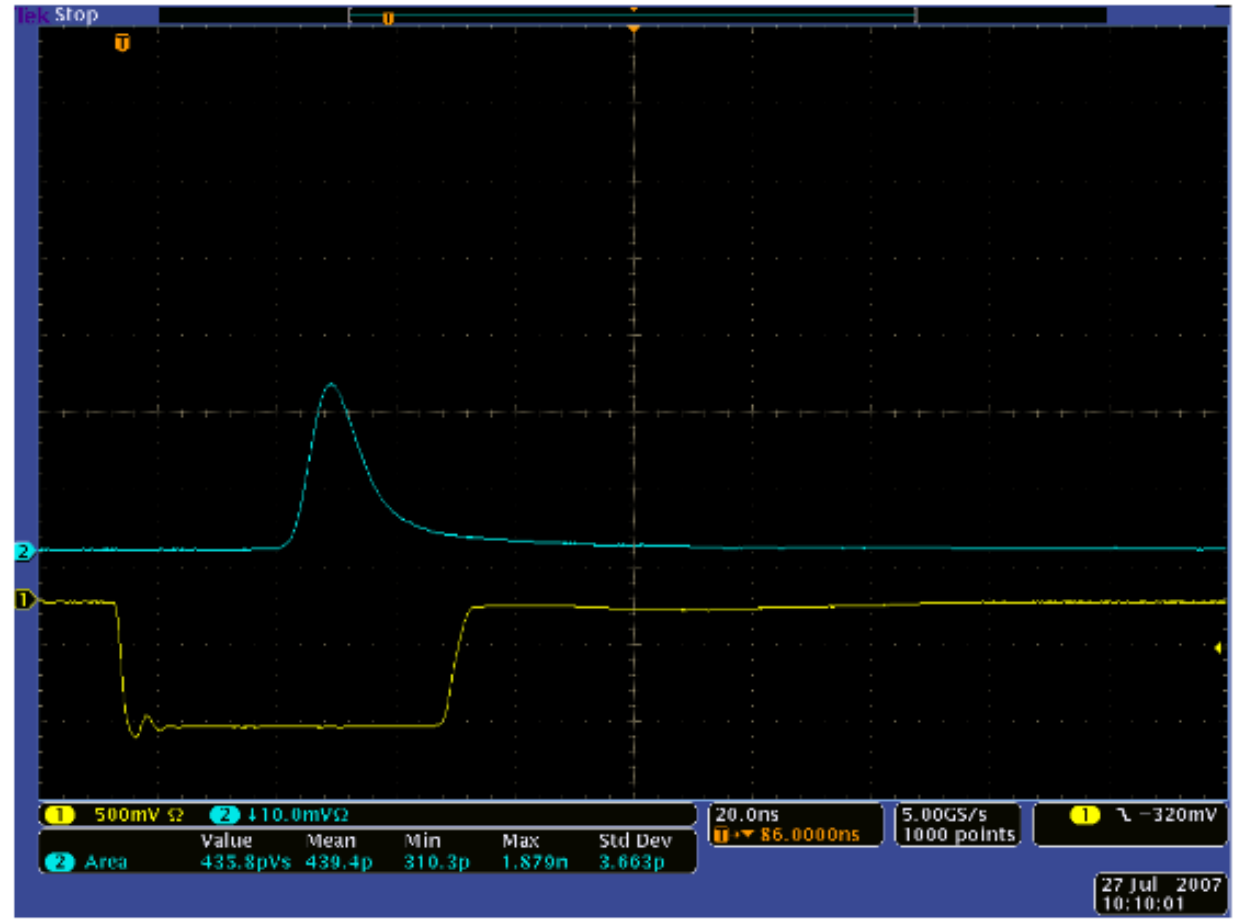

DPO4104 - 10:11:20 AM 7/27/2007

Figure 16. Tile/fiber configuration C: the pulse-averaged signal for configuration (blue scope trace). Five hundred and twelve pulses were averaged. The type was EJ204 wrapped in Tyvek ${ }^{\circledR}$. Shifter fiber is DSB1. 


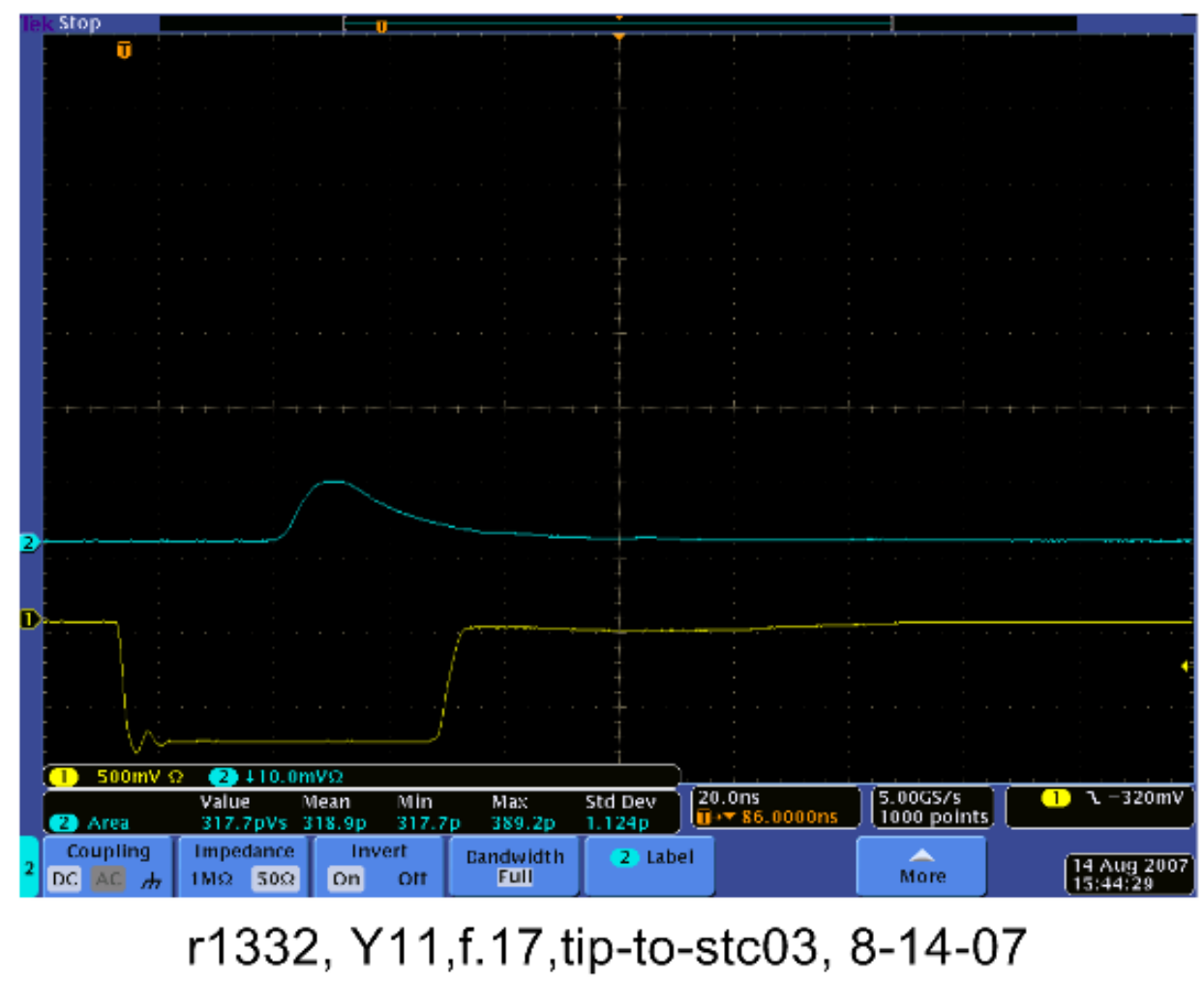

Figure 17. Tile/fiber configuration D: the pulse-averaged signal for configuration (blue scope trace). Five hundred and twelve pulses were averaged. The type was EJ204 wrapped in Tyvek ${ }^{\circledR}$. Shifter fiber is Y11.

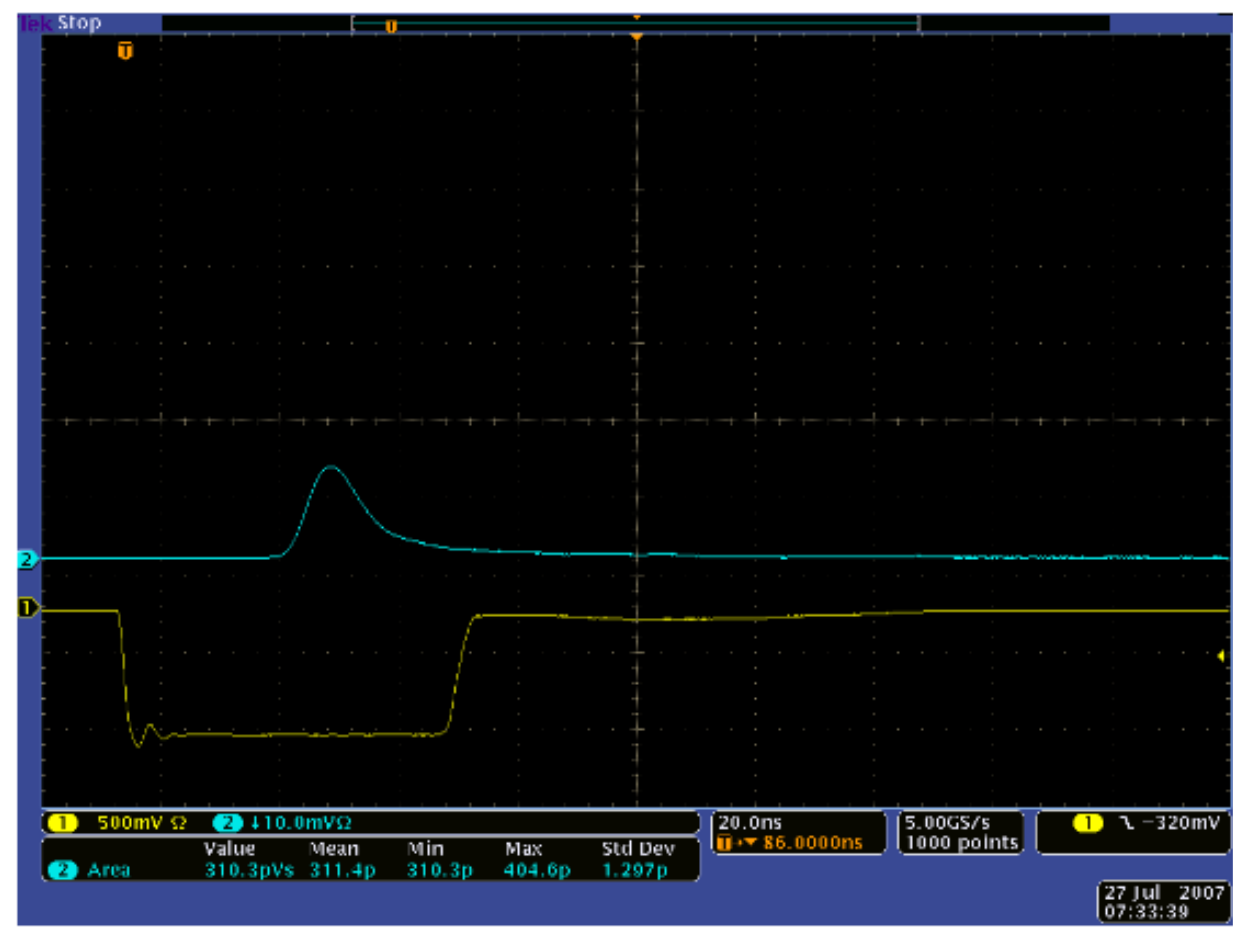

\section{DPO4104 - 7:34:59 AM 7/27/2007}

Figure 18. Tile/fiber configuration D: the pulse-averaged signal for configuration (blue scope trace). Five hundred and twelve pulses were averaged. The type was EJ204 wrapped in Tyvek ${ }^{\circledR}$. Shifter fiber is DSB1. 


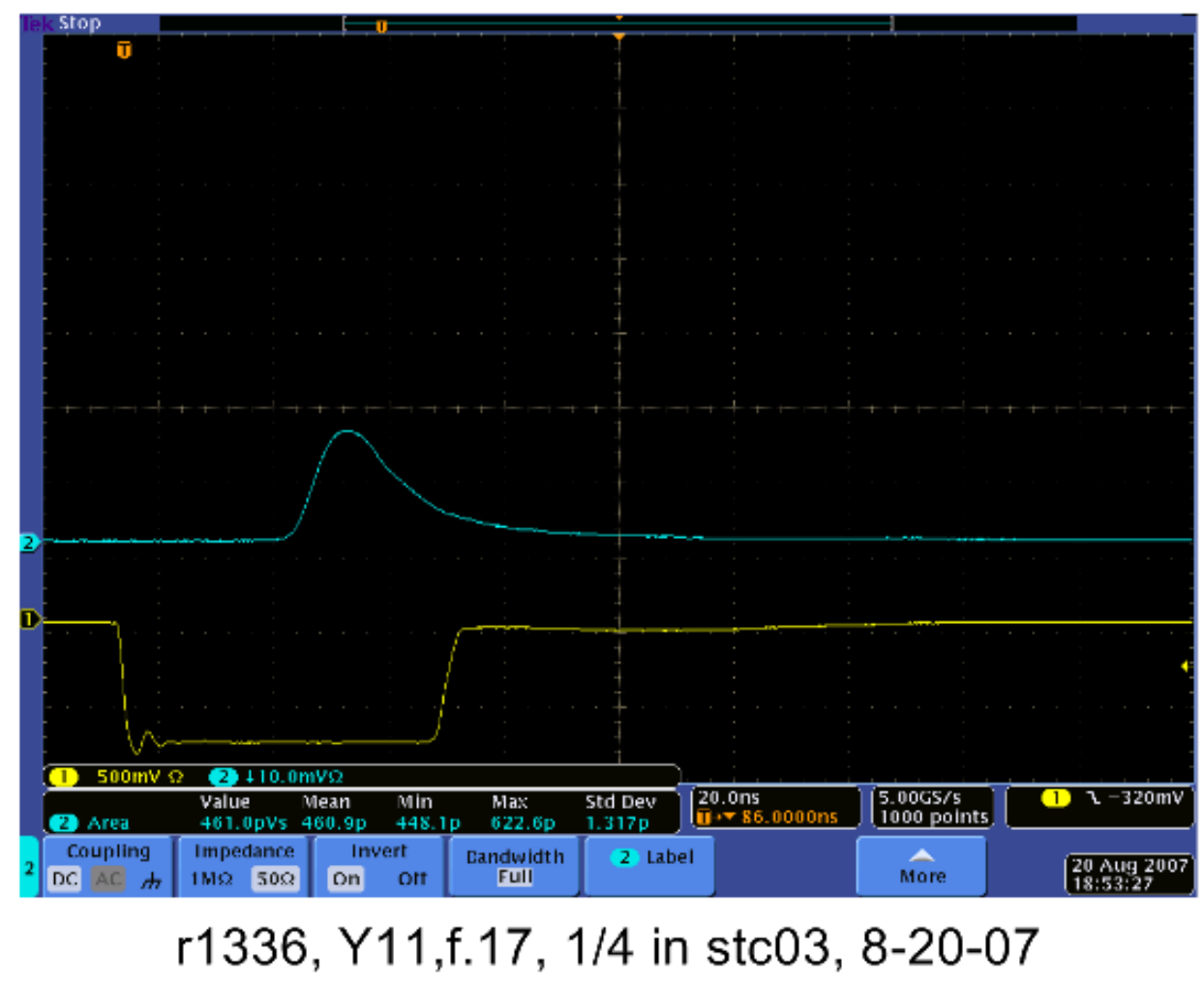

Figure 19. Tile/fiber configuration E: the pulse-averaged signal for configuration (blue scope trace). Five hundred and twelve pulses were averaged. The type was EJ204 wrapped in Tyvek ${ }^{\circledR}$. Shifter fiber is Y11.

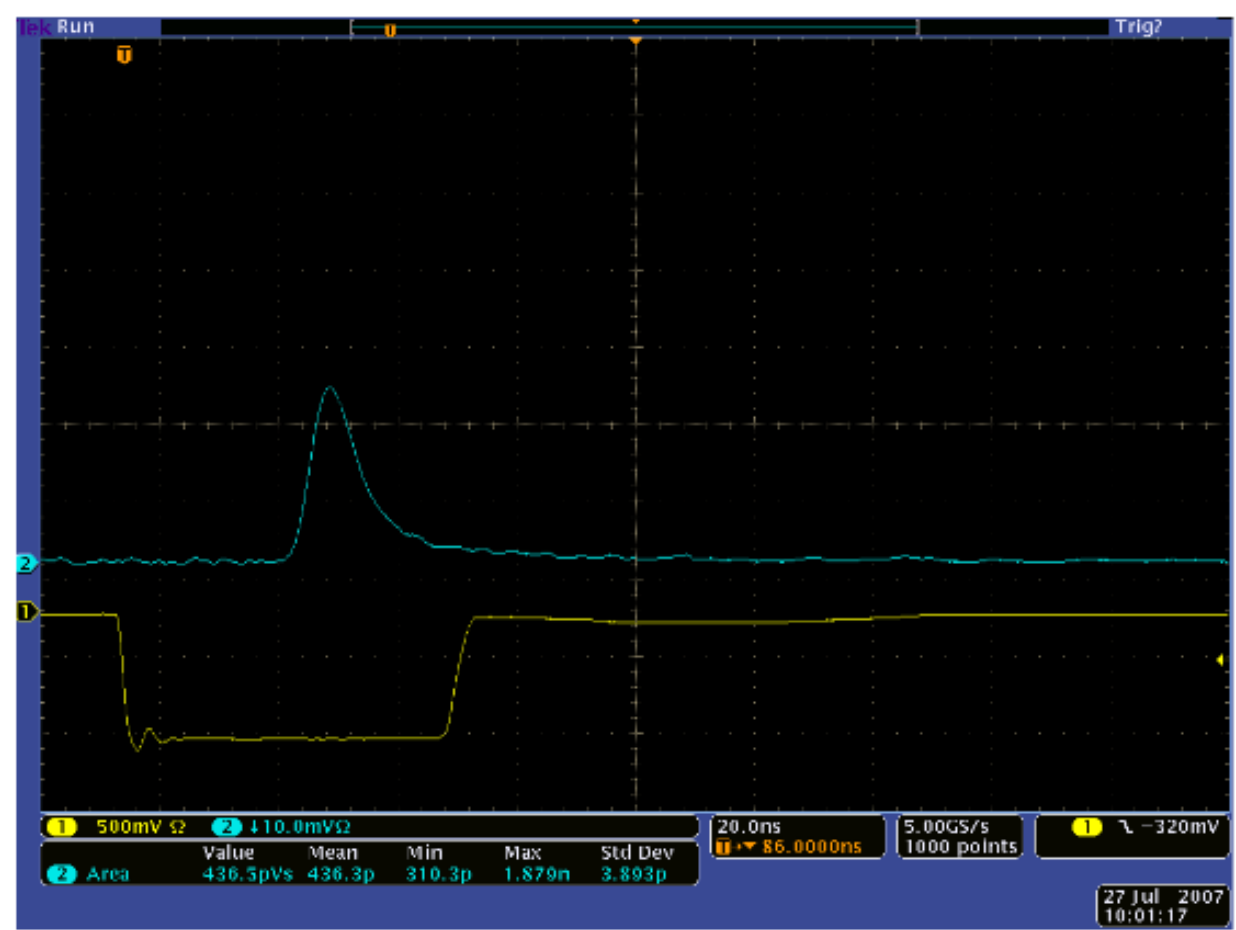

\section{DPO4104 - 10:02:36 AM 7/27/2007}

Figure 20. Tile/fiber configuration E: the pulse-averaged signal for configuration (blue scope trace). Five hundred and twelve pulses were averaged. The type was EJ204 wrapped in Tyvek ${ }^{\circledR}$. Shifter fiber is DSB1. 


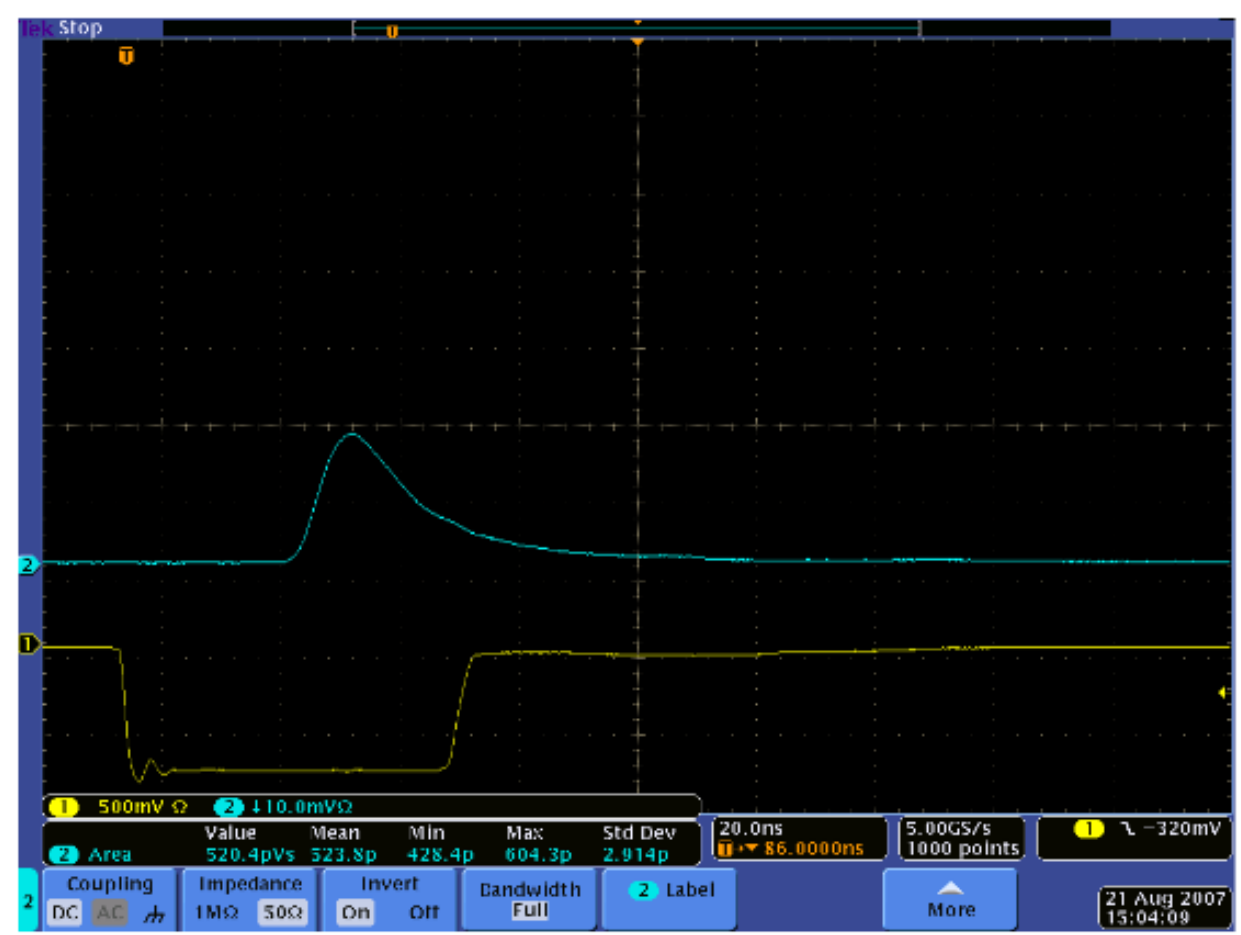

\section{r1337, Y11,f.17, 1/2 in stc03, 8-21-07}

Figure 21. Tile/fiber configuration F: the pulse-averaged signal for configuration (blue scope trace). Five hundred and twelve pulses were averaged. The type was EJ204 wrapped in Tyvek ${ }^{\circledR}$. Shifter fiber is Y11.

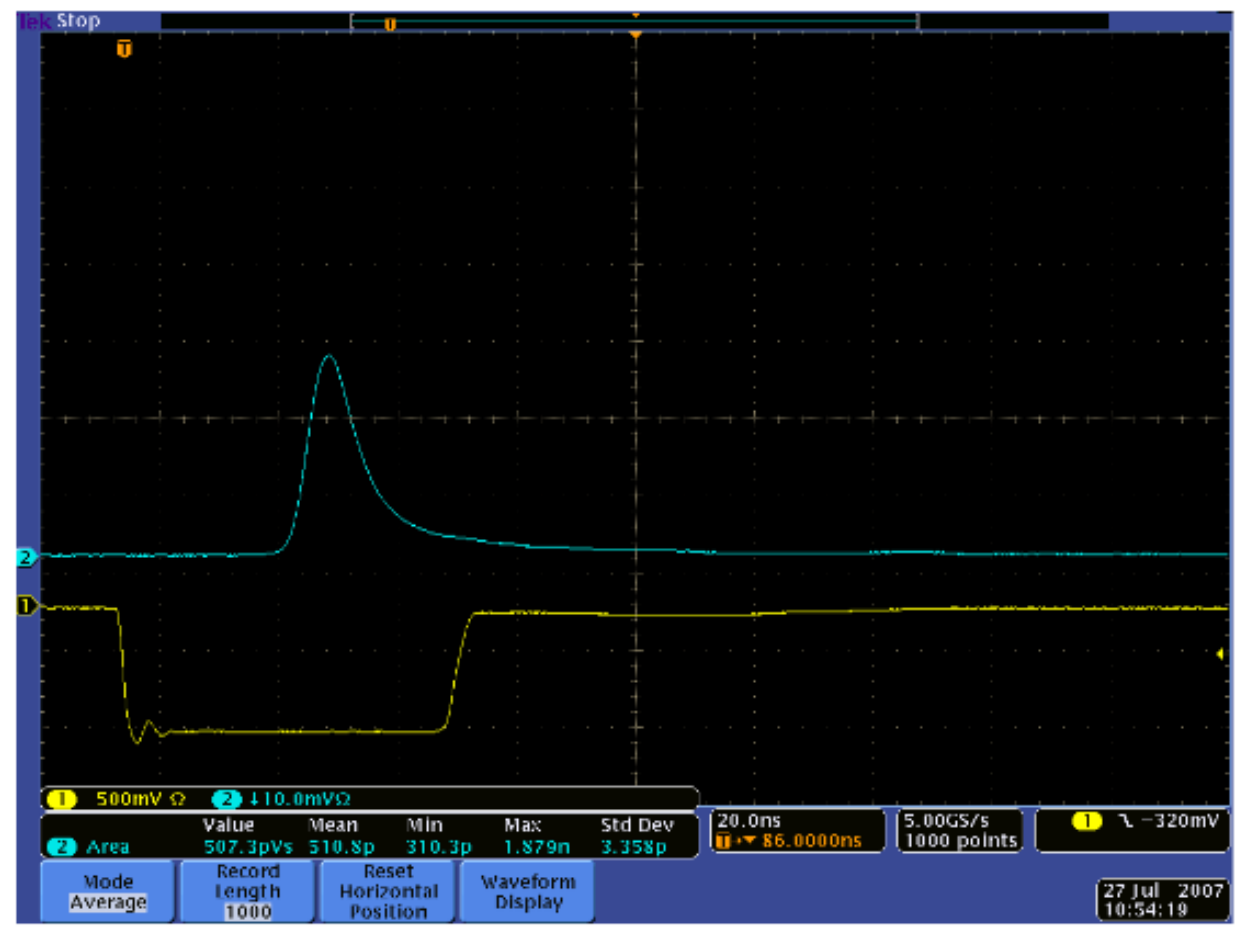

\section{DPO4104 - 10:55:39 AM 7/27/2007}

Figure 22. Tile/fiber configuration F: the pulse-averaged signal for configuration (blue scope trace). Five hundred and twelve pulses were averaged. The type was EJ204 wrapped in Tyvek ${ }^{\circledR}$. Shifter fiber is DSB1. 


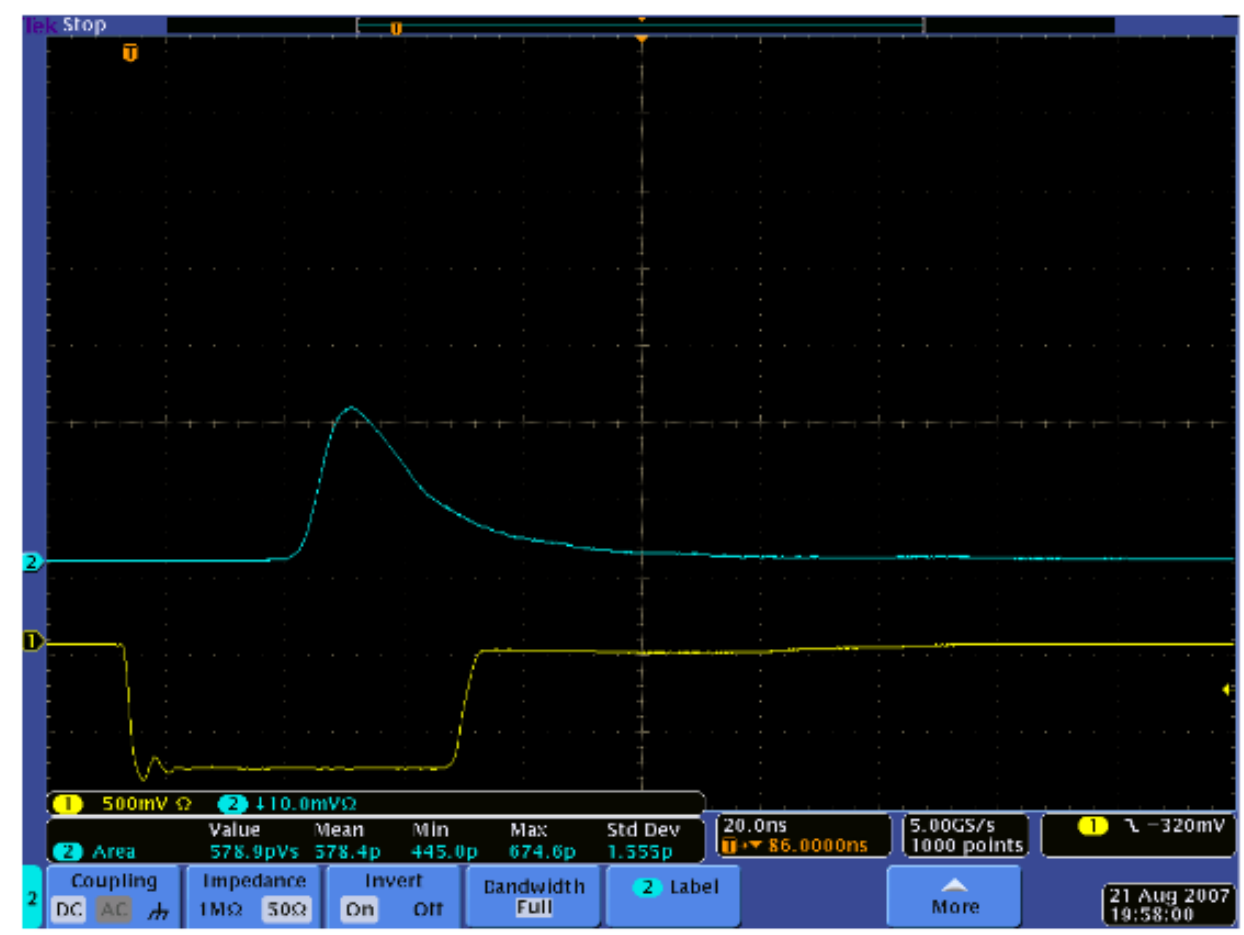

\section{r1339, Y11,f.17, 3/4 in stc03, 8-21-07}

Figure 23. Tile/fiber configuration G: the pulse-averaged signal for configuration (blue scope trace). Five hundred and twelve pulses were averaged. The type was EJ204 wrapped in Tyvek ${ }^{\circledR}$. Shifter fiber is Y11.

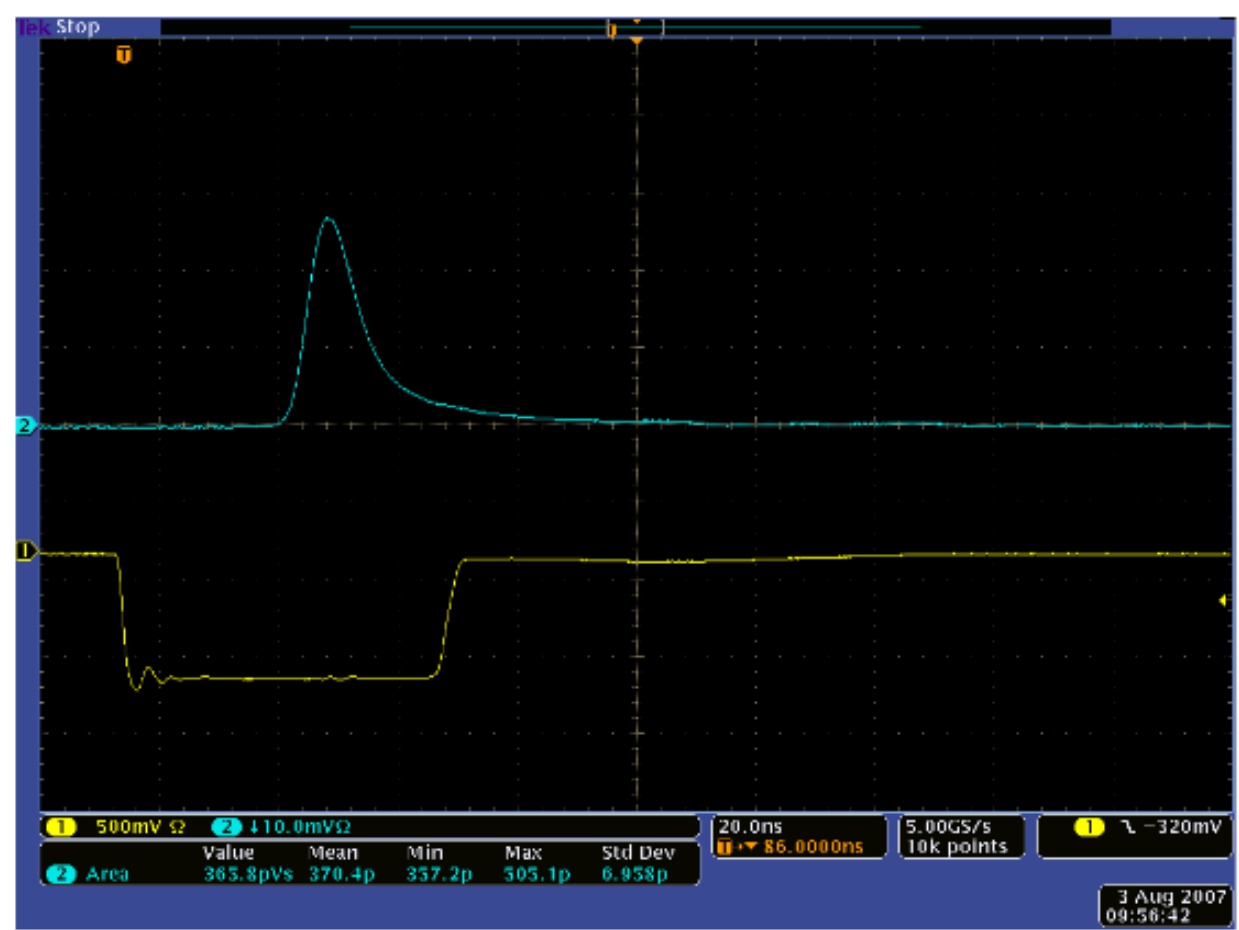

\section{DPO4104 - 9:57:52 AM 8/3/2007}

Figure 24. Tile/fiber configuration G: the pulse-averaged signal for configuration (blue scope trace). Five hundred and twelve pulses were averaged. The type was EJ204 wrapped in Tyvek ${ }^{\circledR}$. Shifter fiber is DSB1. 


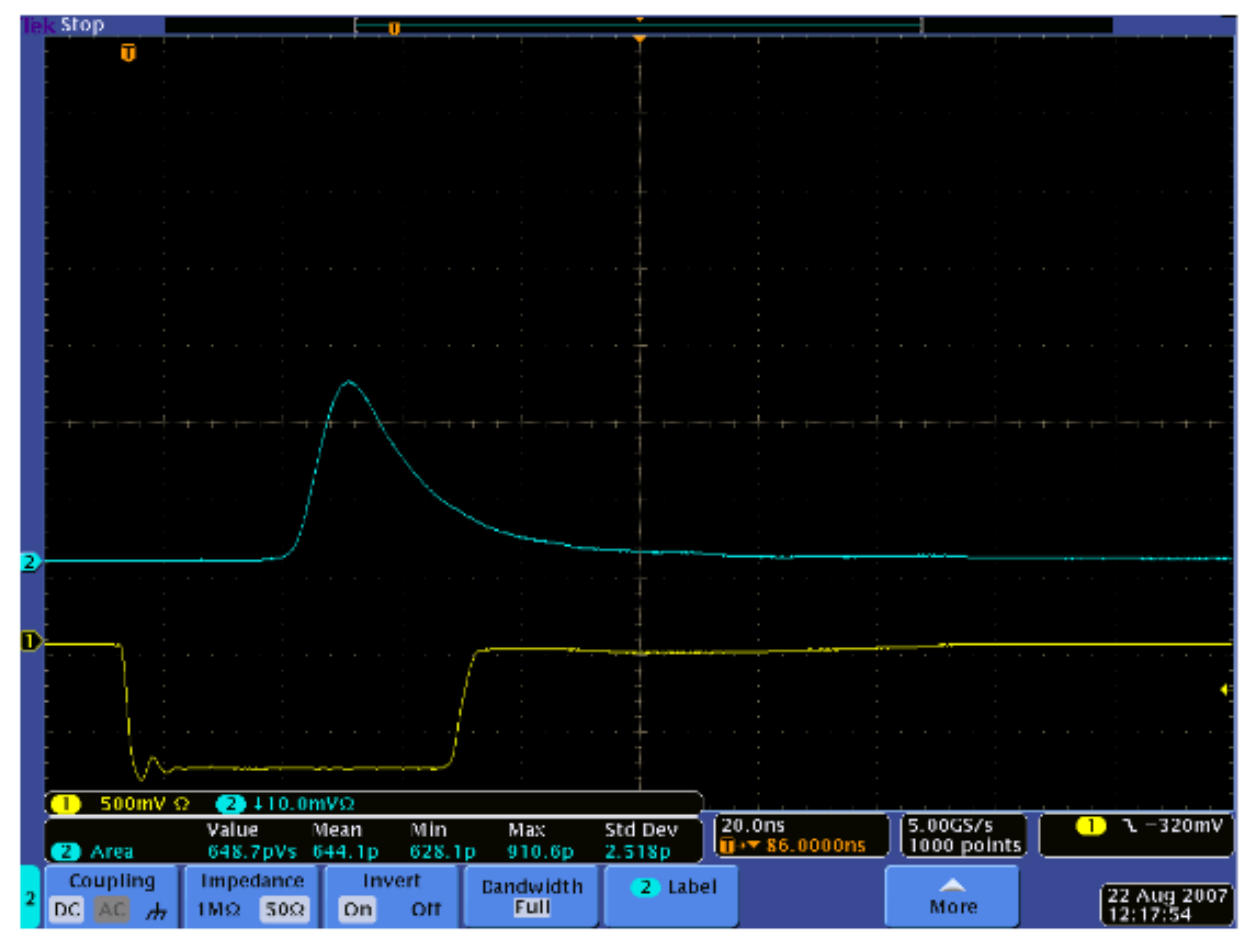

\section{r1340, Y11,f.17, all-in stc03, 8-22-07}

Figure 25. Tile/fiber configuration $\mathrm{H}$ : the pulse-averaged signal for configuration (blue scope trace). Five hundred and twelve pulses were averaged. The type was EJ204 wrapped in Tyvek ${ }^{\circledR}$. Shifter fiber is Y11.

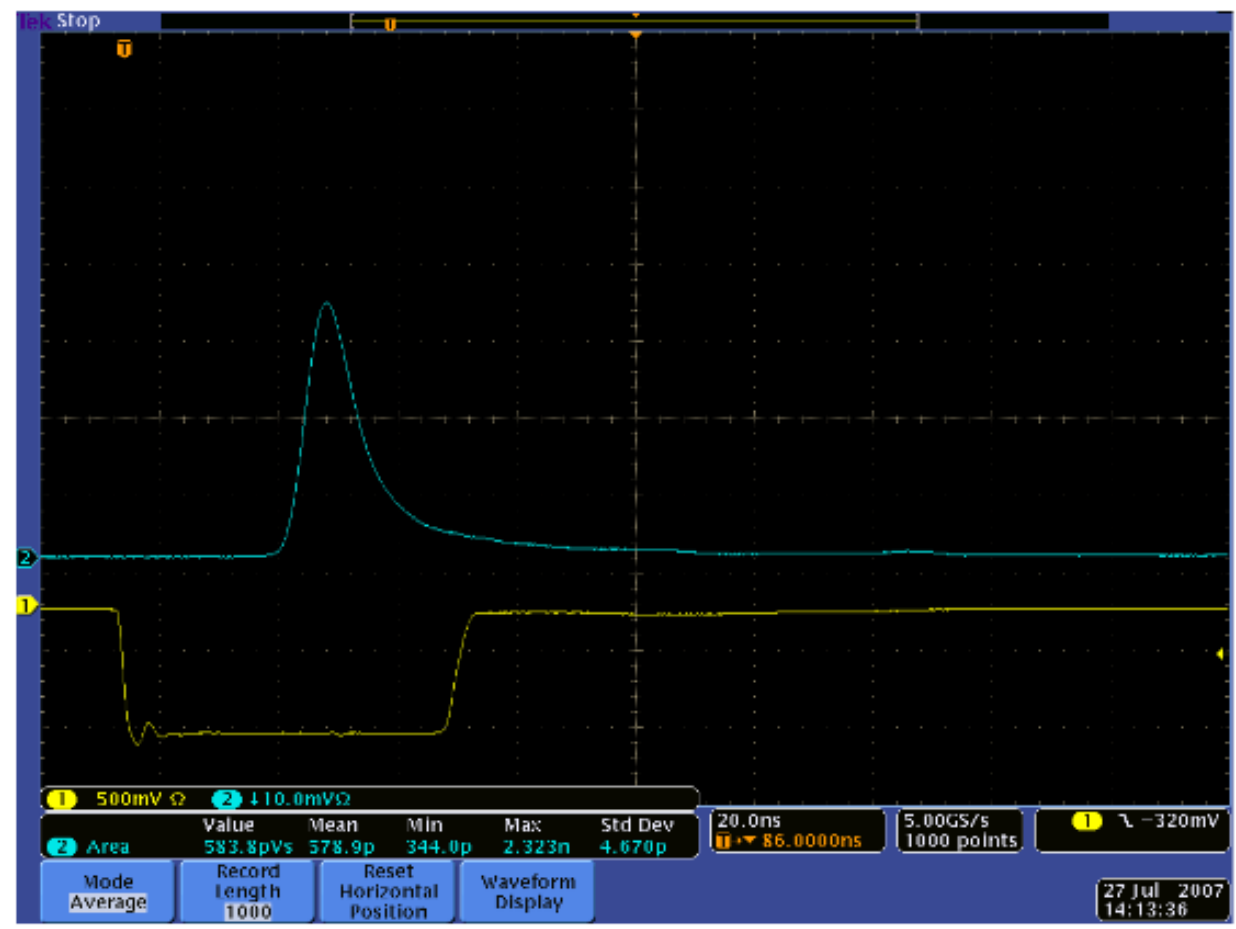

\section{DPO4104 - 2:14:43 PM 7/27/2007}

Figure 26. Tile/fiber configuration H: the pulse-averaged signal for configuration (blue scope trace). Five hundred and twelve pulses were averaged. The type was EJ204 wrapped in Tyvek ${ }^{\circledR}$. Shifter fiber is DSB1. 


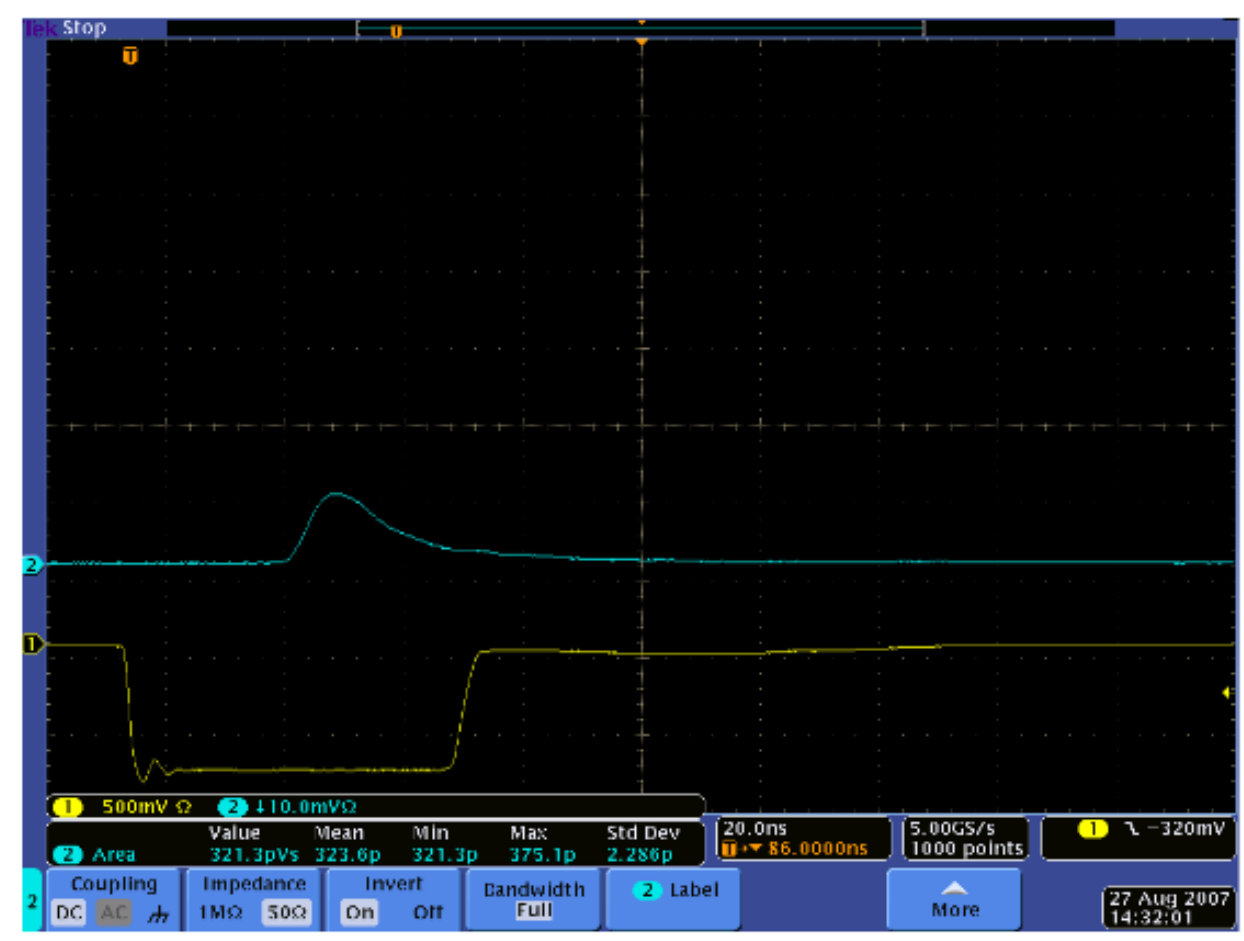

\section{r1347, Y11,f.17 tip-to-stc03, 8-26-07}

Figure 27. Tile/fiber configuration D: the pulse-averaged signal for configuration (blue scope trace). Five hundred and twelve pulses were averaged. The type was EJ204 wrapped in Tedlar ${ }^{\circledR}$. Shifter fiber is Y11.

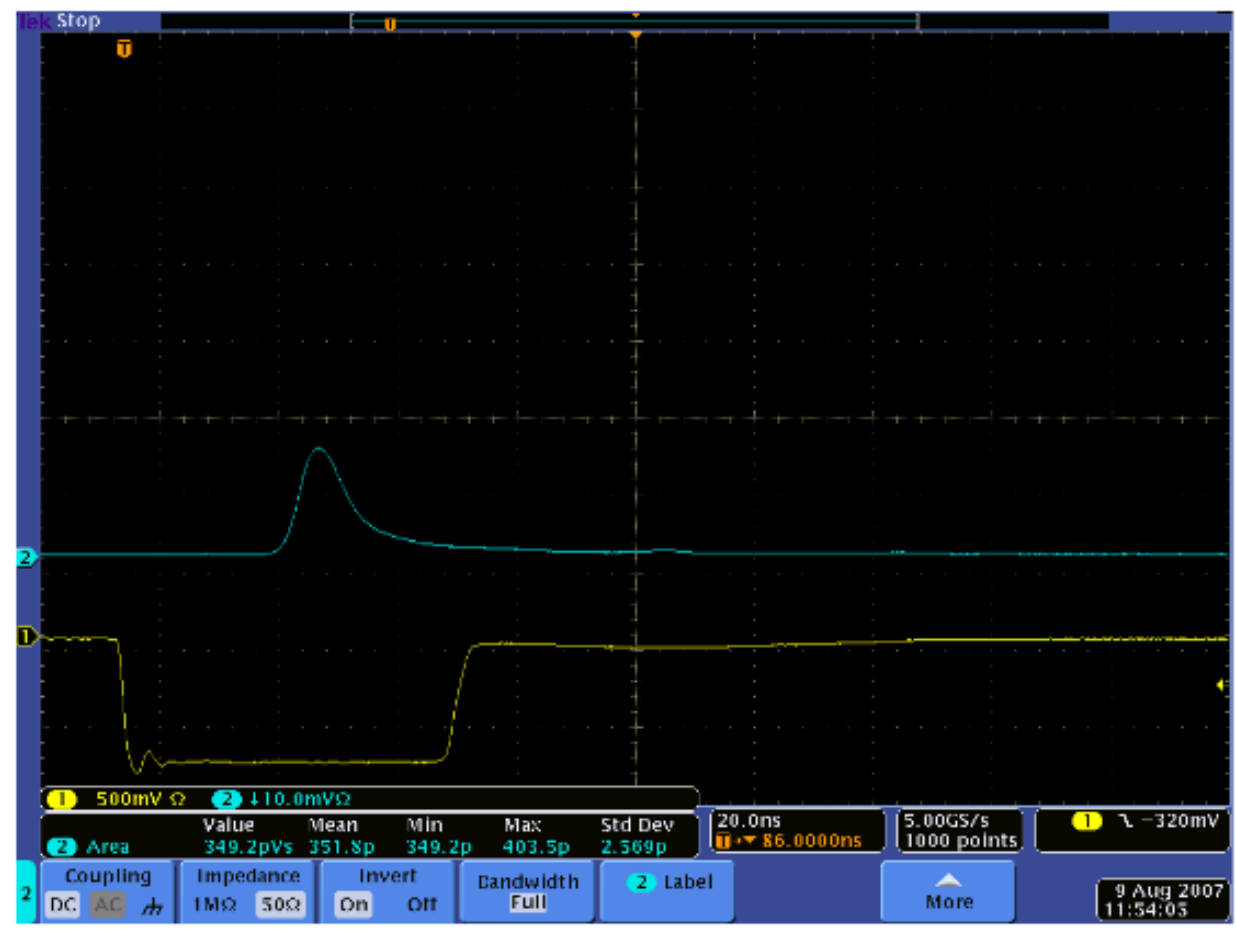

\section{r1329, DSB1,f.22,tip-to-stc03, 8-09-07}

Figure 28. Tile/fiber configuration D: the pulse-averaged signal for configuration (blue scope trace). Five hundred and twelve pulses were averaged. The type was EJ204 wrapped in Tedlar ${ }^{\circledR}$. Shifter fiber is DSB1. 


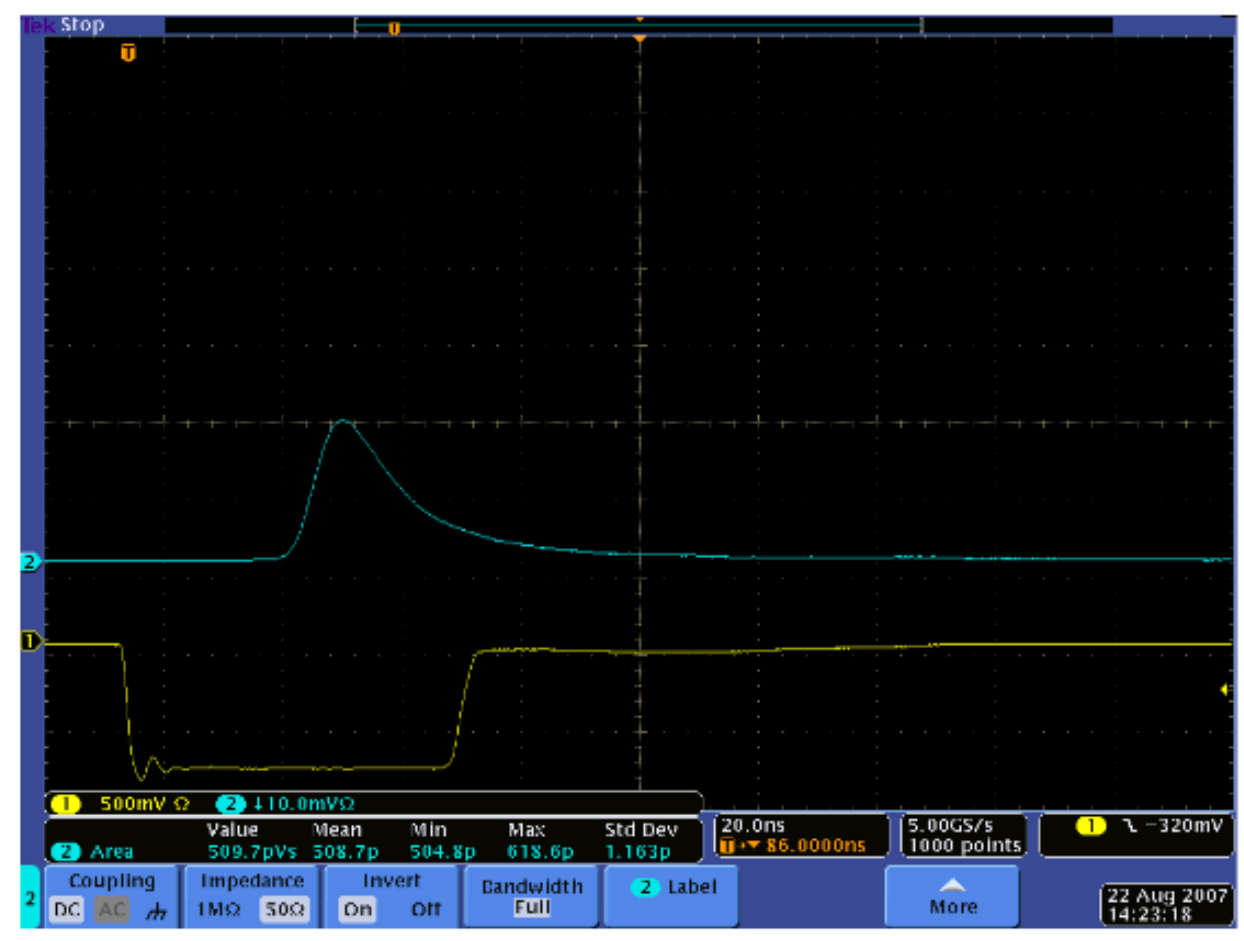

\section{r1341, Y11,f.17, all-in stc03, 8-22-07}

Figure 29. Tile/fiber configuration $\mathrm{H}$ : the pulse-averaged signal for configuration (blue scope trace). Five hundred and twelve pulses were averaged. The type was EJ204 wrapped in Tedlar ${ }^{\circledR}$. Shifter fiber is Y11.

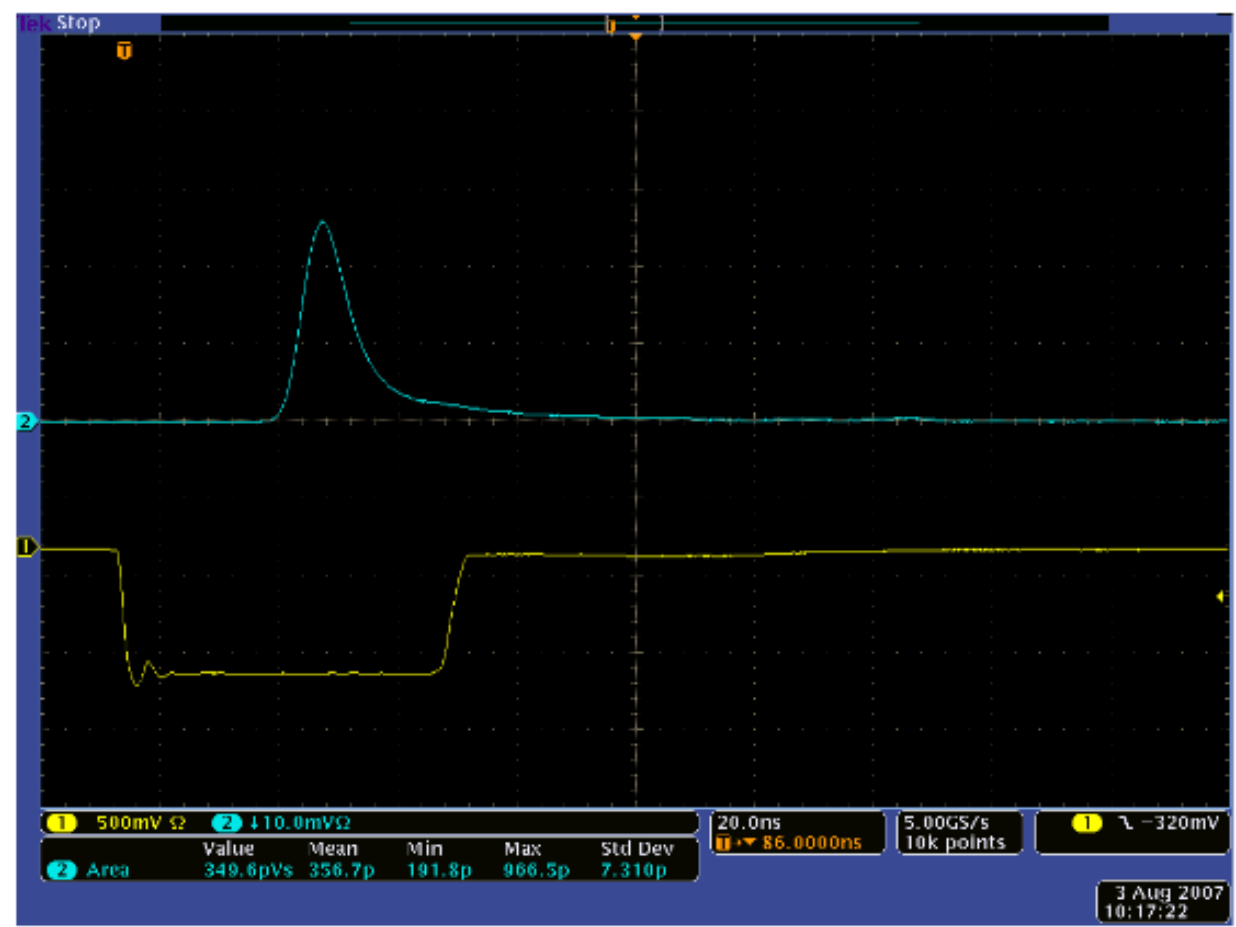

\section{DPO4104 - 10:18:32 AM 8/3/2007}

Figure 30. Tile/fiber configuration H: the pulse-averaged signal for configuration (blue scope trace). Five hundred and twelve pulses were averaged. The type was EJ204 wrapped in Tedlar ${ }^{\circledR}$. Shifter fiber is DSB1. 


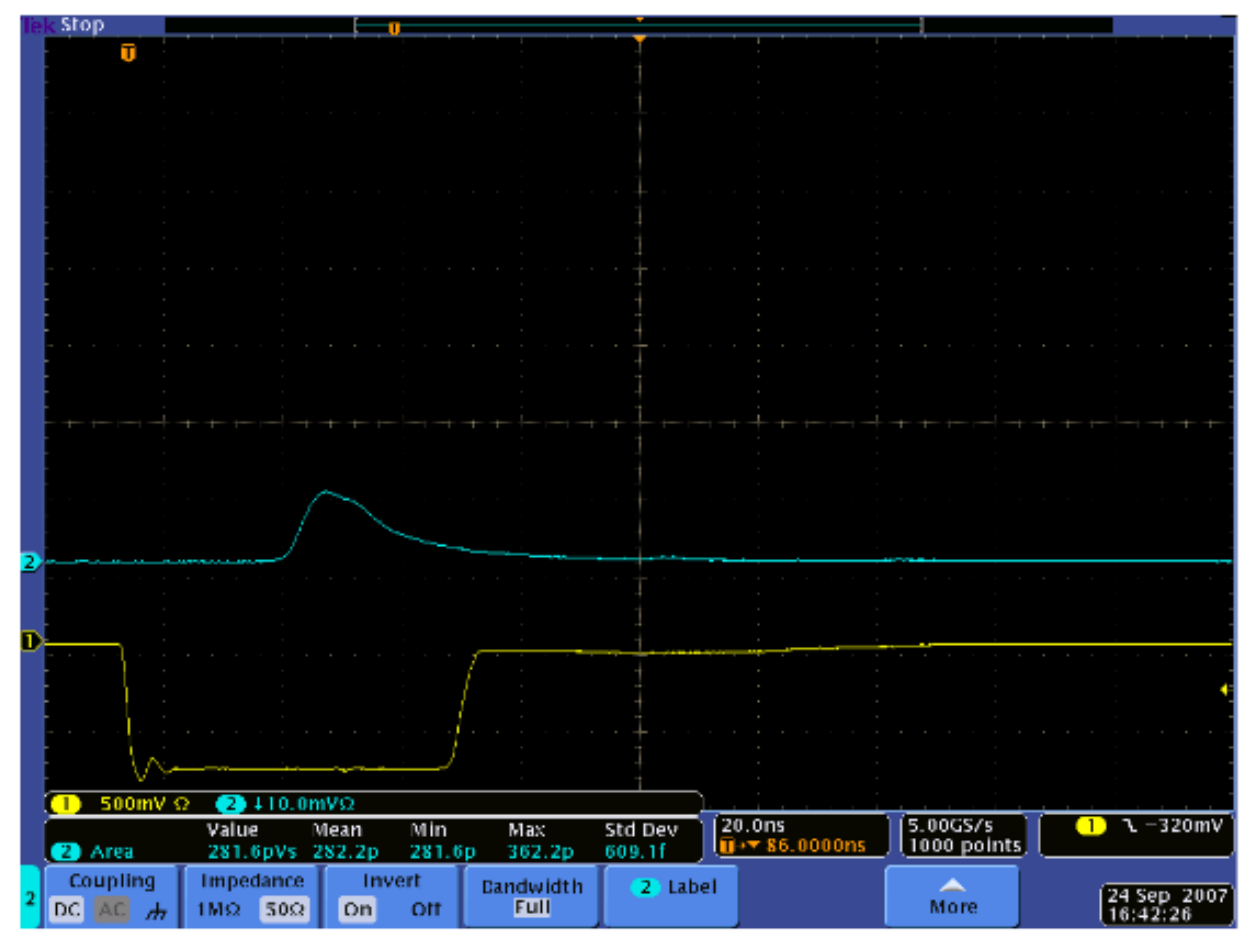

\section{r1384,Y11,f.17,tip-t-stc03,b.c,9-22-07}

Figure 31. Tile/fiber configuration D: the pulse-averaged signal for configuration (blue scope trace). Five hundred and twelve pulses were averaged. The type was EJ204 wrapped in black felt. Shifter fiber is Y11.

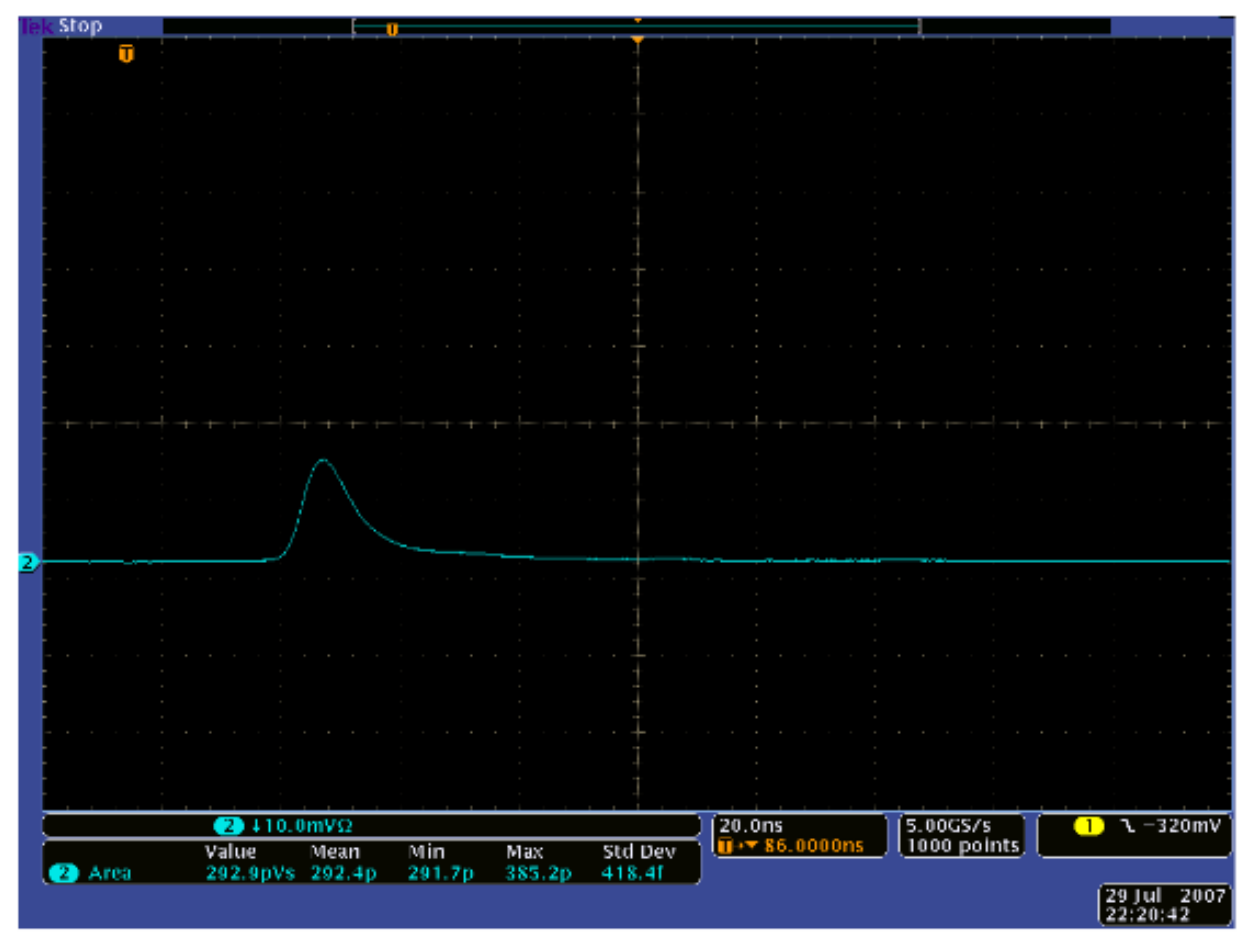

r1313, DSB1,f.22,tip-to-stc03,07-30-07

Figure 32. Tile/fiber configuration D: the pulse-averaged signal for configuration (blue scope trace). Five hundred and twelve pulses were averaged. The type was EJ204 wrapped in black felt. Shifter fiber is DSB1. 


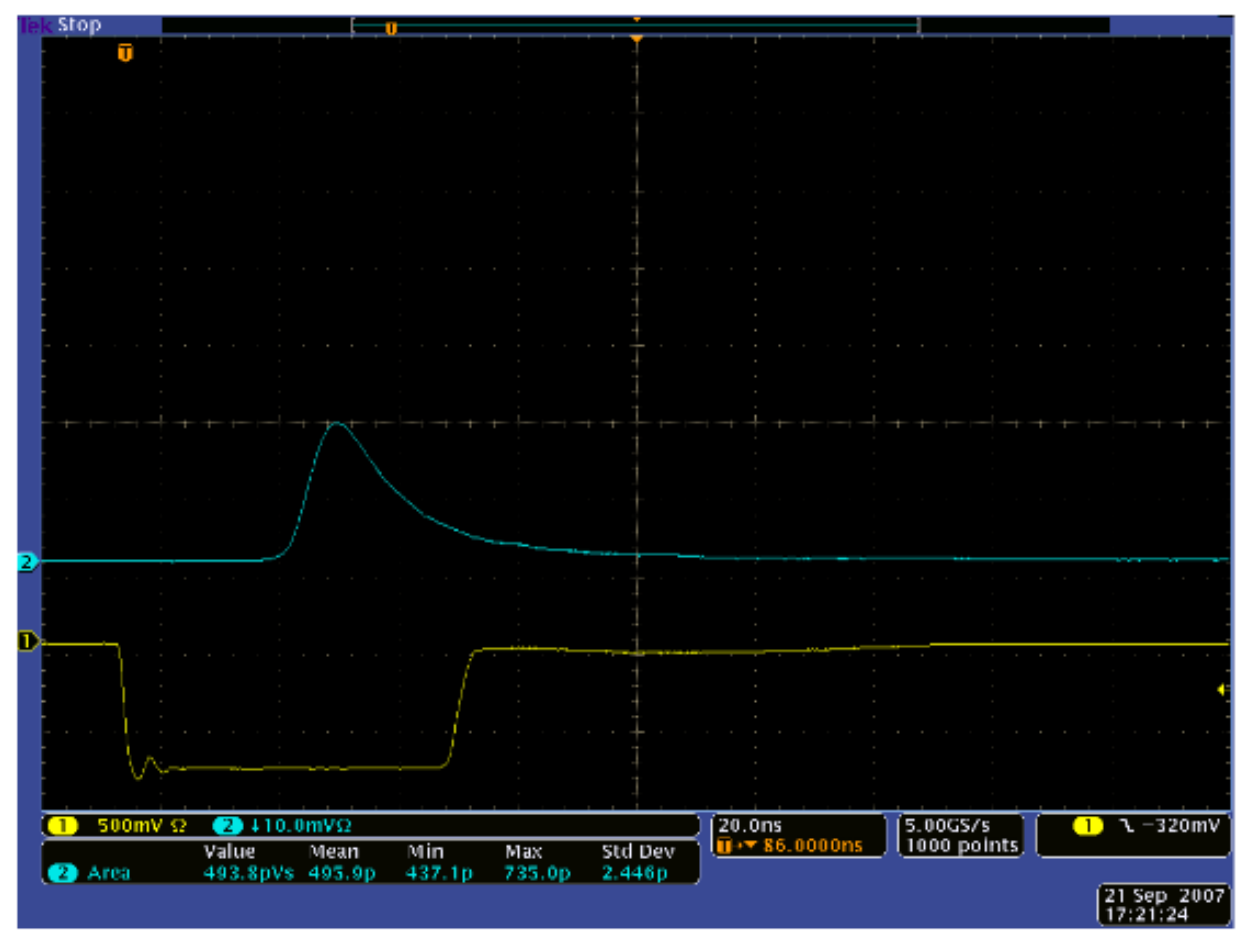

\section{DPO4104 - 5:17:52 PM 9/21/2007}

Figure 33. Tile/fiber configuration H: the pulse-averaged signal for configuration (blue scope trace). Five hundred and twelve pulses were averaged. The type was EJ204 wrapped in black felt. Shifter fiber is Y11.

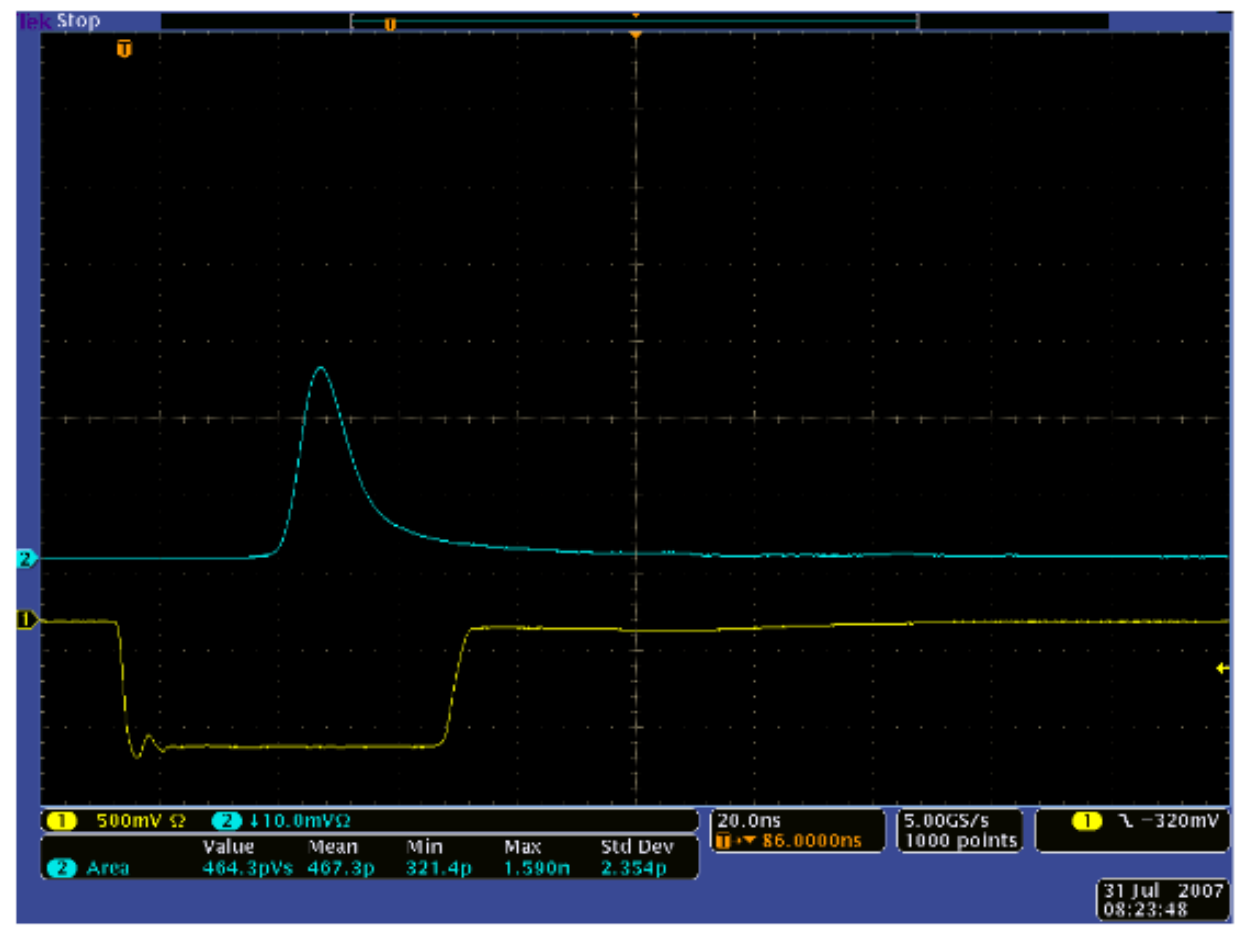

\section{DPO4104 - 8:25:03 AM 7/31/2007}

Figure 34. Tile/fiber configuration H: the pulse-averaged signal for configuration (blue scope trace). Five hundred and twelve pulses were averaged. The type was EJ204 wrapped in black felt. Shifter fiber is DSB1. 
One observes striking changes in these studies. Upon averaging of 512 pulses:

1. DSB1 shifter exhibits a narrower (in time) and higher (in amplitude) response over Y11. We associate this with more rapid fluorescence decay and a greater molar extinction coefficient for the dye material.

2. As the waveshifter fiber is moved from edge on excitation (configuration $\mathrm{H}$ ) to end-on excitation (configuration D), the excitation moves from more light yield to less light yield, and from off-axis, higher-mode rays (and, hence, the possibility of delayed excitation) to near meridianal rays - and, hence, prompt excitation.

3. Comparing the Tyvek ${ }^{\circledR}$ covering to Tedlar ${ }^{\circledR}$ and to black felt, the overall light level is reduced with the latter two materials, but the effective average pulse width relative to pulse amplitude remains unchanged, indicating that multiple scintillation light bounces in the tile are also potentially responsible for delayed excitation, and also create pulse jitter of any excitation. This effect can be seen in Figures 35 and 36, which display individual pulses (rather than averaged signals) for configuration D for both Y11 and DSF1 waveshifters.

There are some scenarios that we would like to explore further:

1. Adding additional clear fiber onto the waveshifter in varying lengths to see if this reduces the number of delayed pulses of any type (due to the additional path length for optical absorption and due to the presence of additional boundary encounters - related to the reflection coefficient).

2. Use of a fiber connector (ferule) and optical couplant. Such connectors tend to transmit meridianal rays with high efficiency, and higher-mode rays with reduced efficiency.

These scenarios are important for any tile/fiber detector for rapid time response and high efficiency. 


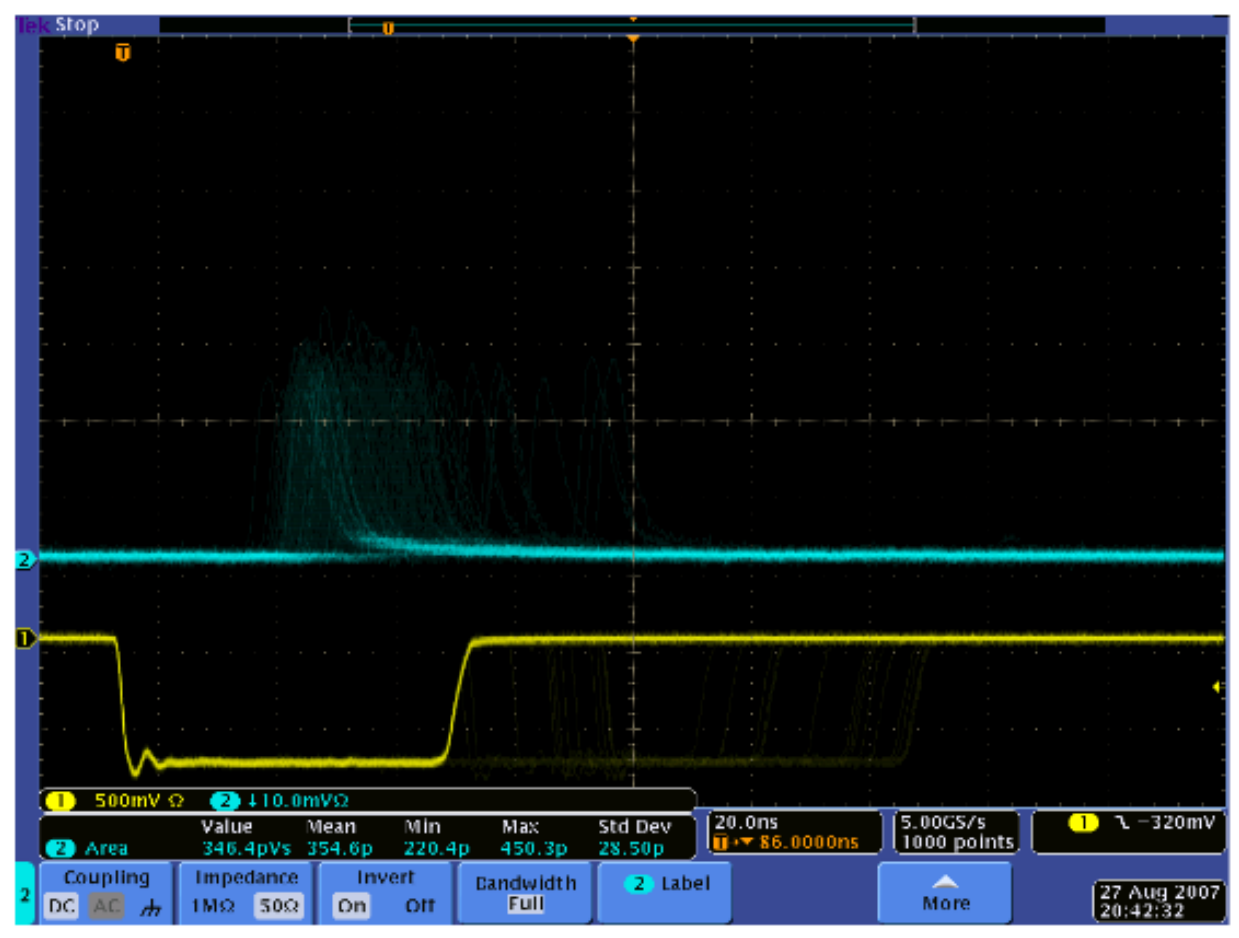

\section{r1347, Y11,f.17 tip-to-stc03, 202 hits}

Figure 35. Tile/fiber configuration D - 202 individual pulses displayed in storage mode (blue scope trace). Timing jitter is clearly seen. The tile was EJ204 wrapped in Tedlar ${ }^{\circledR}$. Shifter fiber is Y11.

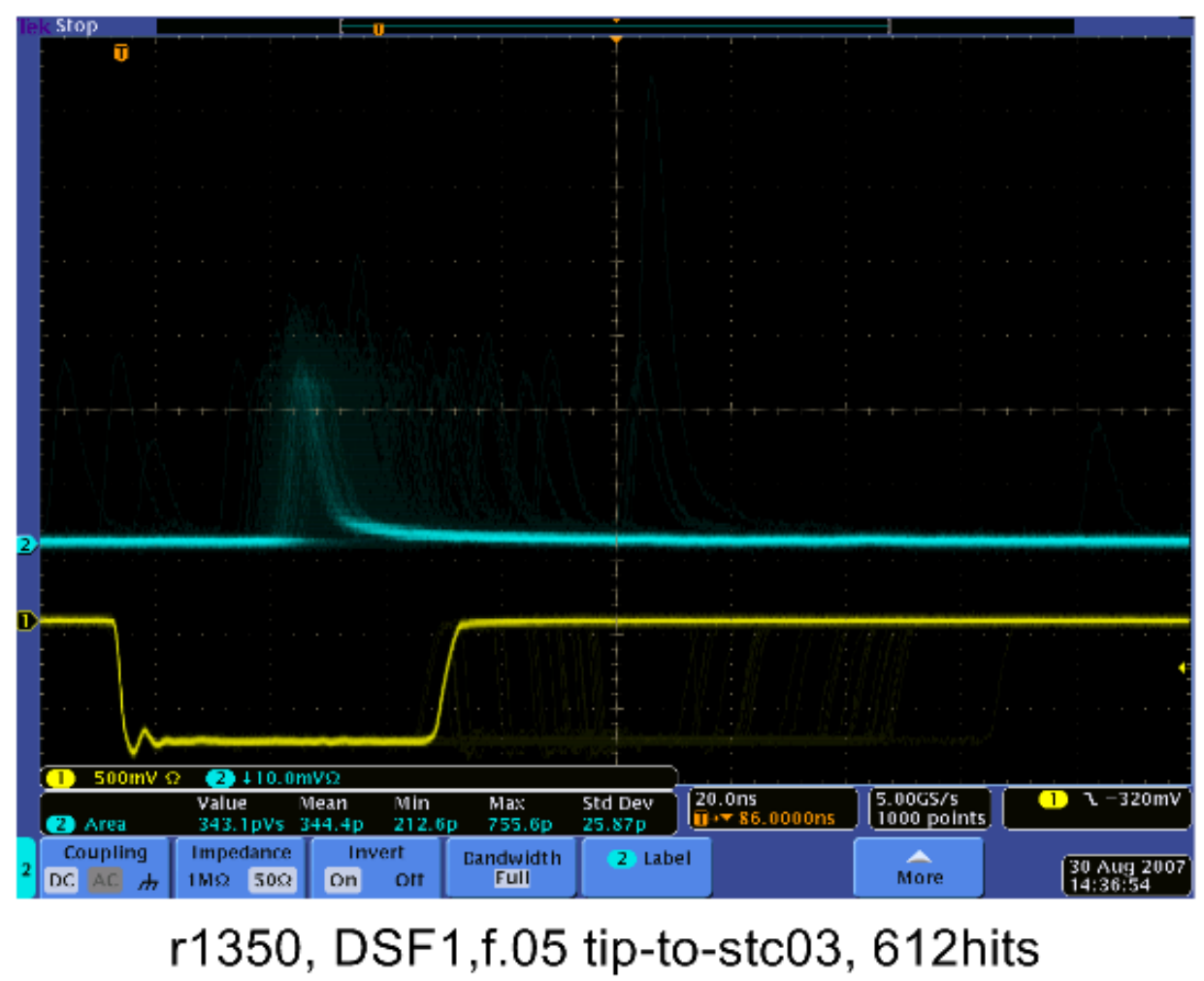

Figure 36. Tile/fiber configuration D - 612 individual pulses displayed in storage mode (blue scope trace). Timing jitter is clearly seen. The tile was EJ204 wrapped in Tedlar ${ }^{\circledR}$. Shifter fiber is DSF1. 


\section{Section 5: Scintillating Fiber Studies for Attenuation Length \& Efficiency}

The aim of these studies was to examine the performance, in double-clad fiber, of several fluorescent dye combinations for efficiency and attenuation length. The materials under study included: 3HF fiber newly-fabricated for these tests (labeled 3HF new); 3HF fiber produced approximately a dozen years ago (circa 1995) for the Dzero Experiment at Fermilab (labeled 3HF old); 4CN-HBT fiber produced a dozen years ago (circa 1995) by our group for comparative testing for the Dzero Experiment; and OX-550F newlyfabricated for these tests. All fibers were of $835 \mu \mathrm{m}$ diameter and $3 \mathrm{~m}$ length. The test setup is indicated in Figure 37 and, schematically, in Figure 38, and consists of the fiber under test which is read out with a GaAsP PMT, and two trigger counters above and below the scintillating fiber to provide trigger information that an electron from the ${ }^{90} \mathrm{Sr}$ source is present. The far ends of the fibers were covered with Apeizon to minimize reflection. Figure 39 presents the measurements, and Table II summarizes the findings. Of the fiber samples, new $3 \mathrm{HF}$ produced the most light (was the most efficient) and old $3 \mathrm{HF}$ was the least efficient, indicating deterioration of performance over time. The performance of $4 \mathrm{CN}-\mathrm{HBT}$ and $\mathrm{OX}-550 \mathrm{~F}$ were similar in character. Relative efficiency of the materials at a distance of $1 \mathrm{~m}$ from the PMT is shown in Table II. Additionally the new $3 \mathrm{HF}$ was brighter over the full $3 \mathrm{~m}$ length of the fiber, and the old $3 \mathrm{HF}$ the worst performer. The conclusion of these studies is that $3 \mathrm{HF}$ remains superior in scintillation efficiency, although there is performance deterioration over time. (This circumstance is not unexpected for most materials.) One concern was the amount of time taken to make the measurements and, additionally, the variation in the measurement points. This led to studies of fiber attenuation length characterization using LEDs. 


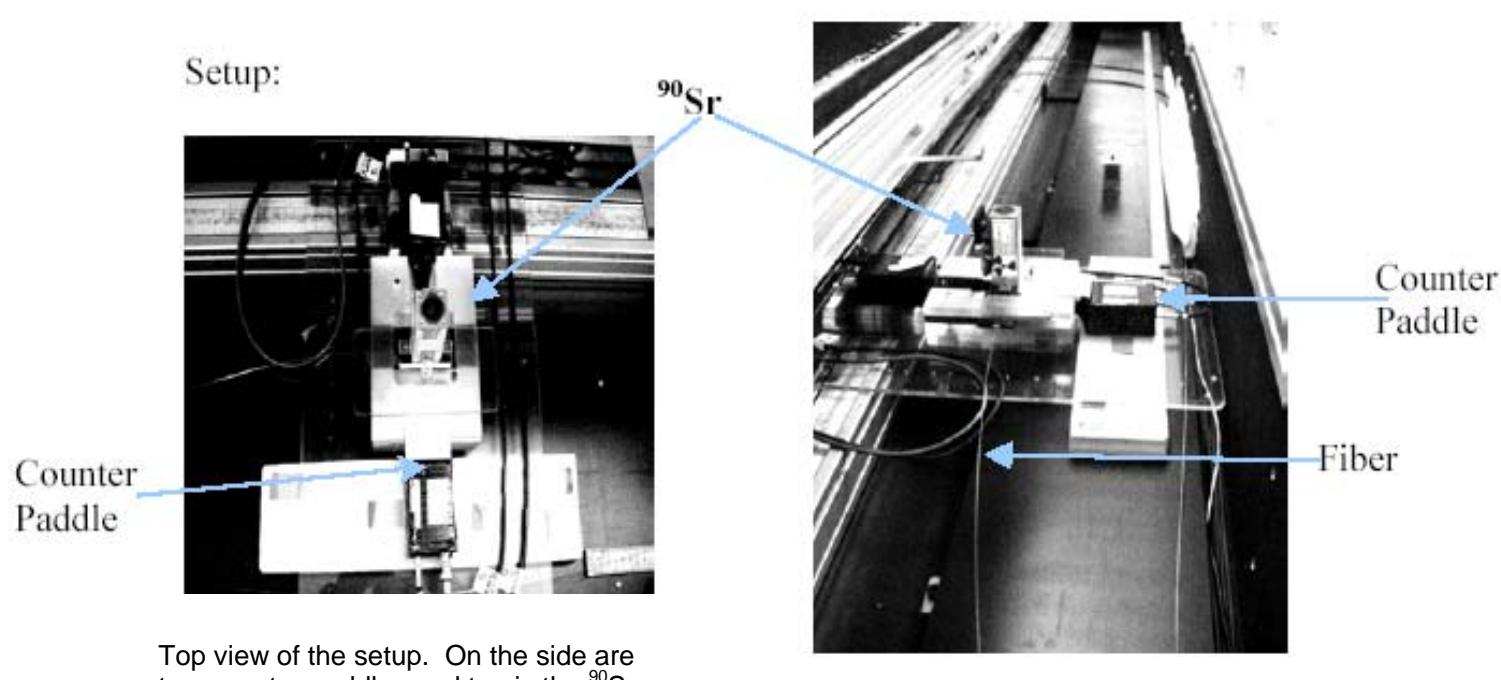
two counter paddles and top is the ${ }^{90} \mathrm{Sr}$.

Side view of the setup. ${ }^{90} \mathrm{Sr}$ is placed above the brass plate that is located between the counter paddles.

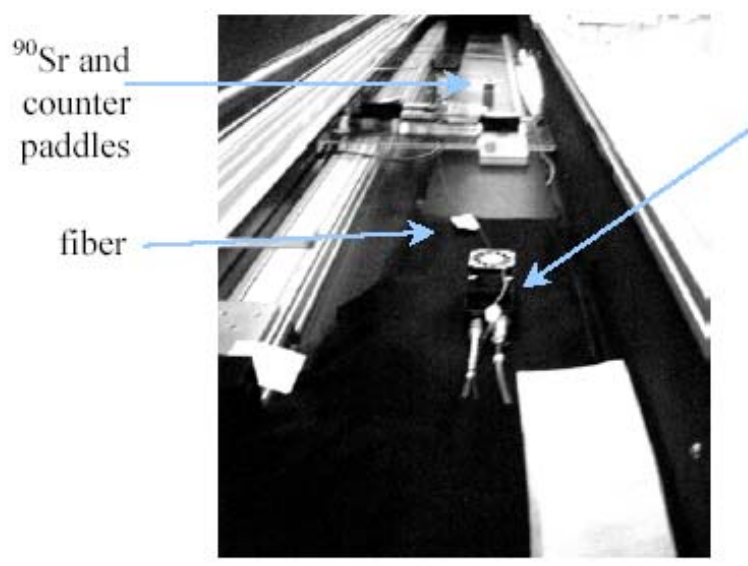

Photomultiplier tube

View from the other end of the box. On one end of the box, the fiber is inserted in the PMT. On the other side, the fiber is placed under the ${ }^{90} \mathrm{Sr}$ and between the counter paddles.

Figure 37. Various views of the geometric arrangement of the scintillating fiber tests for efficiency and attenuation length. Excitation of the system was via electrons from a ${ }^{90} \mathrm{Sr}$ source. Trigger counters were placed above and below the fiber under test and the optical signal from the fiber was read out with a GaAsP PMT.

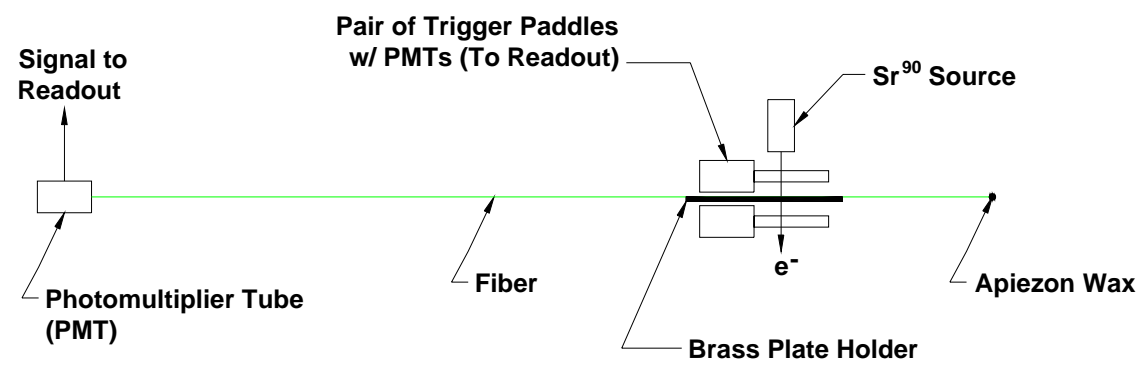

Figure 38. Schematic of the arrangement of the scintillating fiber tests for efficiency and attenuation length. Excitation of the system was via electrons from a ${ }^{90} \mathrm{Sr}$ source. Trigger counters were placed above and below the fiber under test and the optical signal from the fiber was read out with a GaAsP PMT. 


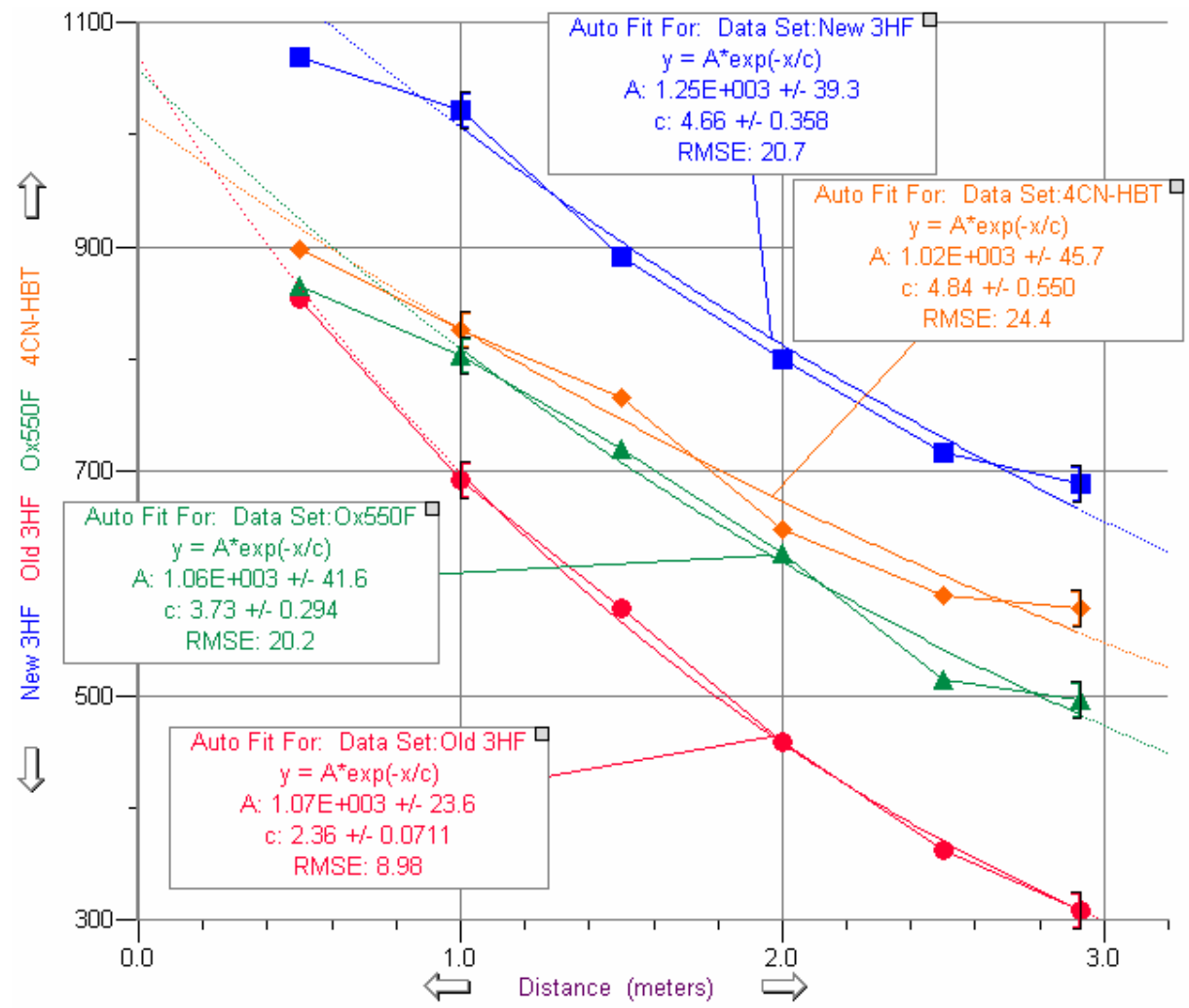

$(2.006,391.4)$

Figure 39. Attenuation length measurements for several scintillating fibers excited with a ${ }^{90} \mathrm{Sr}$ beta source: New 3HF (blue); 4CN-HBT (yellow); OX-550F (green); and Old 3HF (red). The new 3HF fiber is a brighter scintillator at all distances measured. Photosensor is a GaAsP PMT.

TABLE II

RELATIVE EFFICIENCY OF SCINTILLATING FiBER MATERIALS

WITH ${ }^{90}$ SR SOURCE PLACED AT 1 AND 3 METERS FROM THE PMT. FAR END OF THE FIBER IS BLACKENED WITH APIEZON ${ }^{\circledR}$.

\begin{tabular}{|l|l|l|l|}
\hline Material & $\begin{array}{l}\text { Light Yield } \\
\text { Relative to 3HF } \\
\text { new (Source at } \\
\text { 1m from PMT) }\end{array}$ & $\begin{array}{l}\text { Light Yield } \\
\text { Relative to 3HF } \\
\text { new (Source at } \\
\text { 3m from PMT) }\end{array}$ & $\begin{array}{l}\text { Attenuation } \\
\text { Length as } \\
\text { measured from } \\
\text { Figure 39 }\end{array}$ \\
\hline 3HF new & 1.0 & 1.0 & $4.66 \mathrm{~m}$ \\
\hline 3HF old & 0.68 & 0.45 & $2.36 \mathrm{~m}$ \\
\hline 4CN-HBT & 0.81 & 0.84 & $4.84 \mathrm{~m}$ \\
\hline OX-550F & 0.78 & 0.72 & $3.73 \mathrm{~m}$ \\
\hline
\end{tabular}

Given the observed degradation of performance of the $3 \mathrm{HF}$ fiber material over time, and also noting that source measurements of the fibers are very time-consuming, a variation on the fiber testing method was attempted. Rather than using radioactive source excitation, LEDs of various wavelengths were studied for characterizing attenuation as a function of excitation wavelength.

Since all of the secondary dyes are ESIPT material, all have significant Stokes' Shift and the materials provide little light if excited in the "valley" between excitation and emission 
bands. The advent of short wavelength blue and near UV emitting LEDs offers a potentially simple way to excite the fibers. With a LED wavelength near $370 \mathrm{~nm}$, the ESIPT dyes can be directly excited. With a LED wavelength near $330 \mathrm{~nm}$, the primary dye (pTP or butyl-PBD) can be excited. With a LED wavelength near $270 \mathrm{~nm}$, the polymer of the fiber itself (polystyrene) can be excited. Hence, in principle, this can afford a way to assess the full chain of the scintillation process.

This procedure is now in progress and under systematic study. LEDs of a variety of wavelengths were used to excite the fibers, starting with long wavelengths and progressing toward successfully shorter wavelengths. Figure 40 shows a schematic of the test arrangement and Table III shows the measurements of each material as a function of LED wavelength.

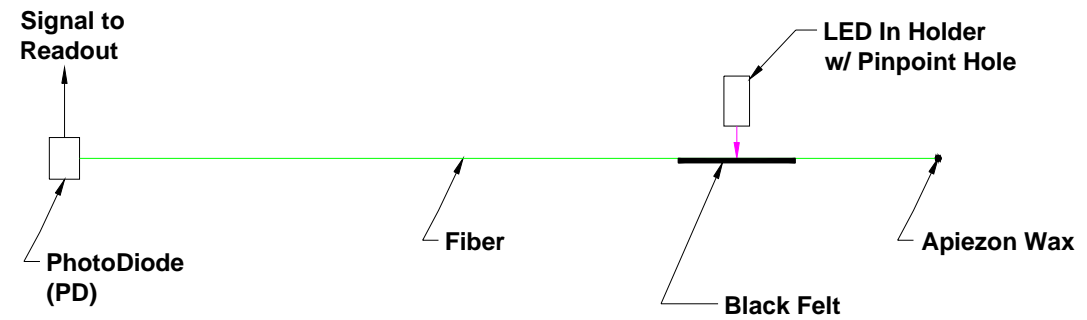

Figure 40. Schematic of the alternative arrangement of the scintillating fiber tests of attenuation length. Excitation of the system was via LEDs of different wavelengths. Readout is via PIN diode and picoammeter.

TABLE III

AtTENUATION LeNGTh of Fibers EXCITED by LEDS of DifFERENT WAVELENGTH. DETECTOR IS A PHOTODIODE WITH PICOAMMETER READOUT. FAR END OF THE FIBER IS BLACKENED WITH APIEZON ${ }^{\circledR}$.

\begin{tabular}{|l|r|r|r|r|}
\hline LED (wavelength) -> & 370nm & 395nm & 400nm & 405nm \\
\hline Atten. Length (m) & & & & \\
\hline 4CN-HBT & 5.42 & 6.25 & 6.19 & 5.86 \\
\hline OX-550F & 4.55 & 4.73 & 4.24 & 4.88 \\
\hline New 3HF & 4.71 & 5.1 & 4.18 & 4.97 \\
\hline Old 3HF & 2.89 & 2.92 & 2.87 & 3.04 \\
\hline
\end{tabular}

With the exception of $\mathrm{OX}-550 \mathrm{~F}$, this measured behavior is reasonably consistent with the ${ }^{90} \mathrm{Sr}$ measurements of Figure 39 and Table II, at the excitation wavelength of $370 \mathrm{~nm}$. There is a variance observed with OX-550F, and this is as yet unexplained. Further measurements will be needed to understand this. Measurements will be continued at still shorter wavelengths, given availability of LEDs. 


\section{Section 6: Liquid and Paint-on Scintillators}

\subsection{Liquid Scintillators}

The motivation for this series of studies comes from several different directions: first, to develop a new class of materials - not necessarily confined to solid plastics for detector applications; second, under environmental exposure - such as high-radiation fields, such materials might be flushed and easily replaced by fresh material; and, third, there may be some cost benefits to using liquids in certain experimental configurations (for example, in lined tanks or canisters) rather than relying on machined solids with polished surfaces.

The liquids used included pseudocumene and phenylxylylethane (PXE) doped with primaries and/or secondaries as necessary. Most of the very efficient dyes developed for use in polystyrene or polyvinyltoluene scintillators and waveshifters worked similarly well in the liquids. An interesting exceptional case was AH238B - a yellow to red emitter - that dissolved well in both solid and liquid materials. In the liquid the light yield was significant, but in the solids the fluorescence was markedly reduced.

To reveal the variety in emission wavelength, Figure 41 shows several liquid samples (in quartz cuvettes) excited by a black light to reveal their final emission as seen by a digital camera.

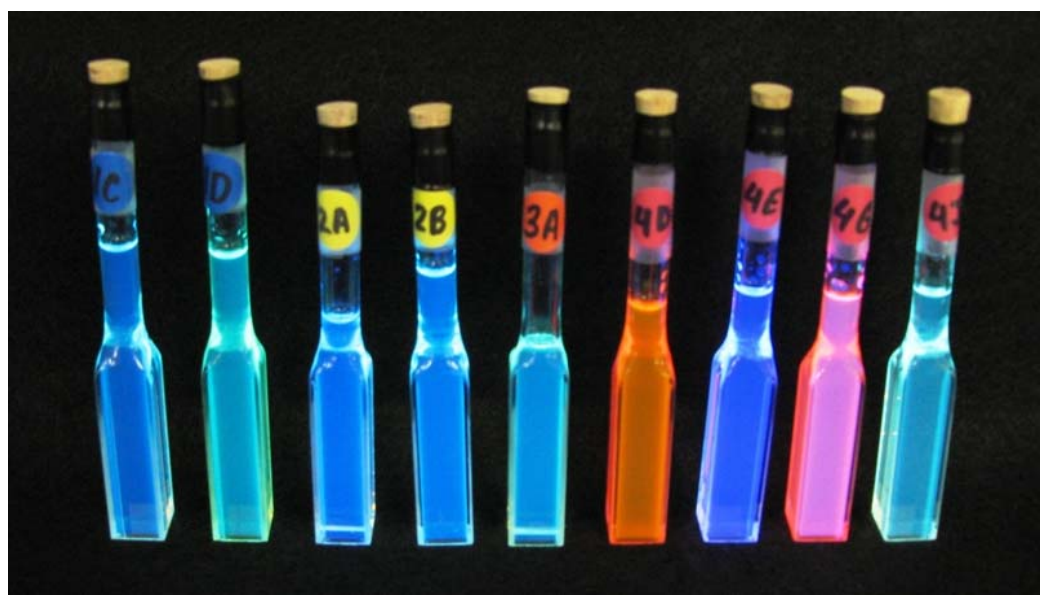

Figure 41. Several liquid scintillator samples in quartz cuvettes, excited under a black light. Left to right: Sample 1C (PXE + PPO + DSB1); Sample 1D (PXE + PPO + DSB2); Sample 2A (Pseudocumene + PPO + DSF1); Sample 2B (PXE + PPO + DSF1); Sample 3A (Pseudocumene + PPO + DSF2); Sample 4D (PXE + AH238B); Sample 4E (PXE + BBOT); Sample 4G (PXE + BBOT + AH238B); Sample 4J (PXE + b-PBD + BBQ).

In the direct testing of solids and liquids, the method for these measurements used ${ }^{90} \mathrm{Sr}$ radioactive source excitation of the bulk materials with and without optical filtering. The physical setup for the testing is shown in Figure 42. Schematics of the test equipment for liquids and solids are displayed in Figures 43 and 44. The PMT used was a Hamamatsu R1104 whose cathode sensitivity and quantum efficiency are displayed in Figure 45. While finite and useful for measurement, this PMT does not have the longwave quantum efficiency of the H7422P-40 PMT, and hence significant correction had to be made for fluorescent materials, such as AH238B, that have long-wave emission. Future studies will use the H7422P-40 GaAsP PMT. 


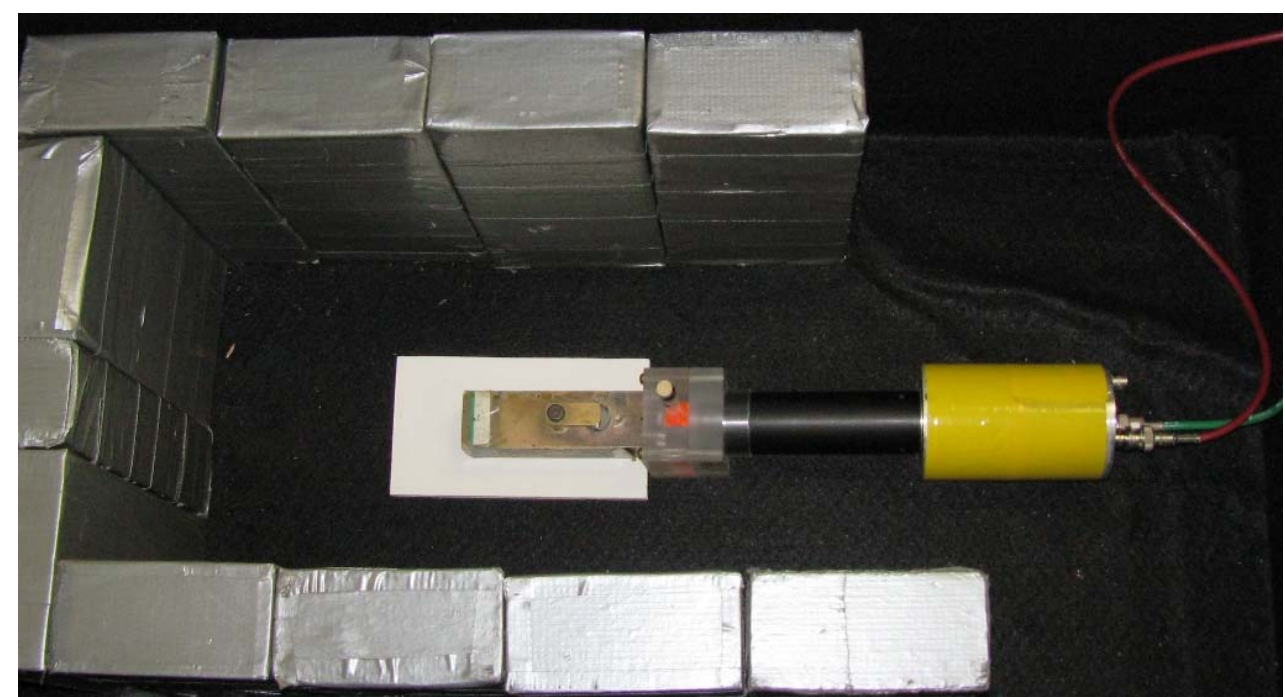

Figure 42. View of the geometric arrangement of the bulk tests of scintillating liquids and solids for efficiency. Excitation of the system was via electrons from a ${ }^{90} \mathrm{Sr}$ source. The photosensor was an R1104 multialkali PMT and readout was via a LeCroy QVT into a computer.

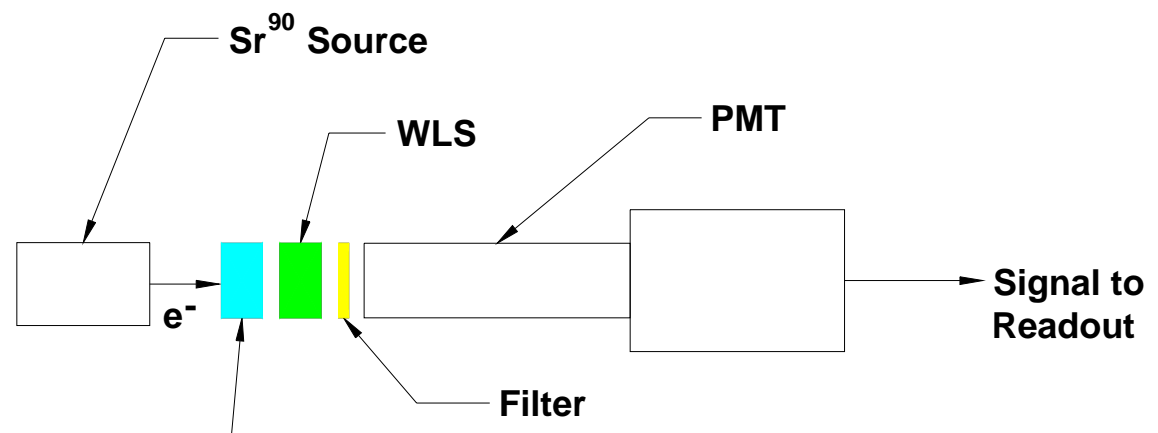

Solid Scintillator

Figure 43. Schematic of the arrangement of the bulk tests of scintillating solids for efficiency. Excitation of the system was via electrons from a ${ }^{90} \mathrm{Sr}$ source. The photosensor was an R1104 multialkali PMT and readout was via a LeCroy QVT into a computer.

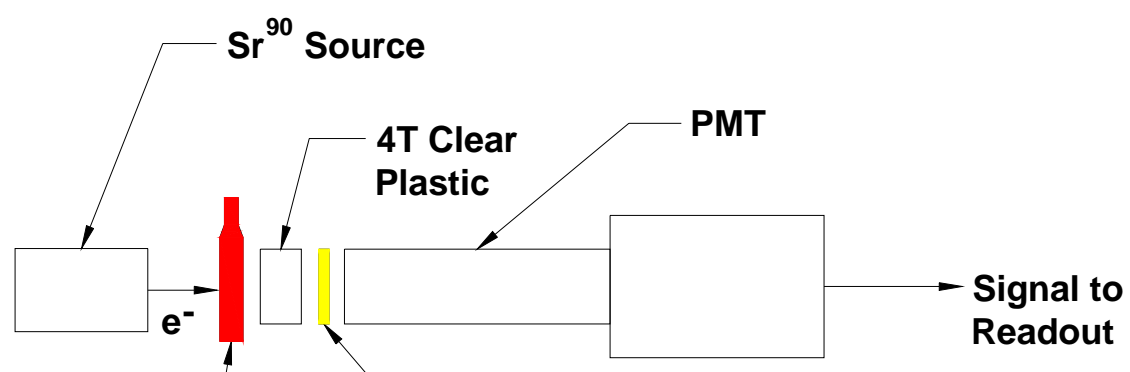

Filter

\section{Liquid Scintillator} in Quartz Cuvette

Figure 44. Schematic of the arrangement of the bulk tests of scintillating liquids for efficiency. Excitation of the system was via electrons from a ${ }^{90} \mathrm{Sr}$ source. The photosensor was an R1104 multialkali PMT and readout was via a LeCroy QVT into a computer. 


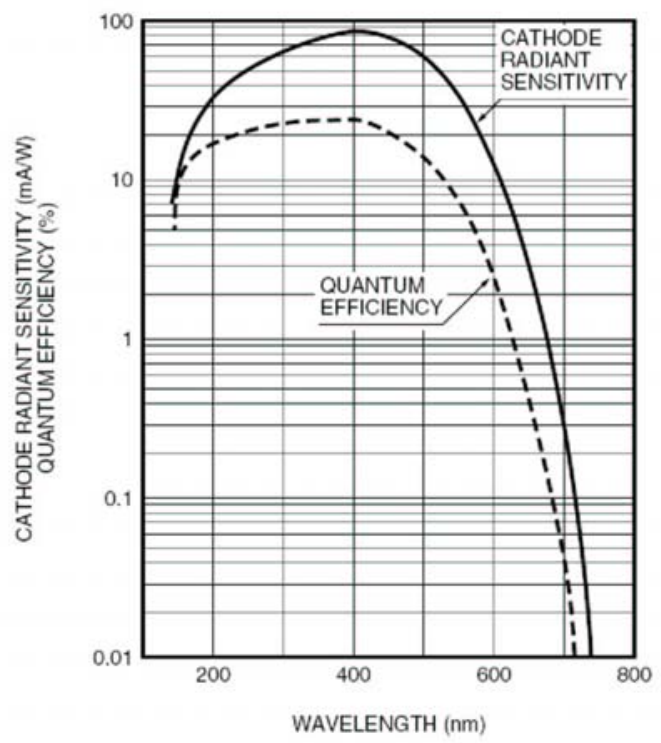

Figure 45. Spectral responsivity and quantum efficiency of the Hamamatsu R1104 PMT. At 500 nm wavelength, the quantum efficiency is approximately $15 \%$ and at $600 \mathrm{~nm}$ the quantum efficiency is of order $2.5 \%$.

The liquids have fluorescence efficiency that is comparable to, or exceeds, that of the plastic materials. Table IV presents the peak emission and the relative efficiency for several of these materials, which are compared to BBQ as the standard. There is a correction factor applied for the quantum efficiency of the R1104 PMT, which is particularly significant in the case of AH238B. One should assume a $>30 \%$ uncertainty in the value of the efficiency of this material relative to BBQ. Nevertheless, the light yield from the AH238B is impressive in the liquid solvent. For Table IV: L1 is PXE and L2 is Pseudocumene; P1, P2, and P3 are BBOT, butyl-PBD and PPO, respectively.

TABLE IV

SCINTILLATION EFFICIENCY OF SAMPLES

$\begin{array}{lllrr}\text { Composition } & \text { Primary } & \text { Secondary } & \begin{array}{r}\text { Emission } \\ \text { Wave- }\end{array} & \begin{array}{r}\text { Efficiency } \\ \text { Relative to } \\ \text { length }(\mathrm{nm})\end{array} \\ \text { Liquid L1 } & \text { Dye } & \text { Dye } & 600 & \begin{array}{r}\text { BBQ } \\ \text { Liquid L1 }\end{array} \\ \text { P1 } & & & 450 & 0.84 \\ \text { Liquid L1 } & \text { P1 } & \text { AH238B } & 600 & 1.05 \\ \text { Liquid L1 } & \text { P2 } & \text { BBQ } & 470 & 1.42 \\ \text { Liquid L1 } & \text { P3 } & \text { DSF1 } & 480 & 1 \\ \text { Liquid L1 } & \text { P3 } & \text { DSB1 } & 500 & 1.02 \\ \text { Liquid L1 } & \text { P3 } & \text { DSB2 } & 510 & 1.11 \\ \text { Liquid L2 } & \text { P3 } & \text { DSF1 } & 480 & 1.08 \\ \text { Liquid L2 } & \text { P3 } & \text { DSF2 } & 480 & 1.02\end{array}$




\subsection{Paint-on Scintillators and Waveshifters:}

The addition of this task is to build upon the opportunity for the development of new fluorescent materials applied in relatively thin layers for applications that involve geometric constraints; the development of new detector structures and the testing of such structures; and the application with specialized photosensors. One example is the current interest in the silicon photomultiplier or SiPM. In general, such devices are relatively efficient at longer wavelengths and less so at short wavelengths. Hence, these devices might benefit from the application of a thin layer of waveshifter to the input face (or entrance window of the device package) to improve light detection at short wavelengths. Another example is the layering of waveshifter materials onto the surface of materials such as quartz, for the shifting of Čerenkov light to longer wavelengths suitable for transmission and detection. Novel applications are also envisaged with layered scintillator and waveshifter materials and tailored for unusual geometries - for example, challenging transition regions in detectors that are generally difficult to instrument.

The scheme is to produce a material which can be applied as a liquid and which dries relatively quickly into a solid, whilst maintaining its fluorescent or scintillation properties. This is a new concept and task to this project and, hence, is just being fleshed out as to its challenges, performance and promise.

Several scintillator and waveshifter "paints" have been produced by Ludlum Measurements, Inc. and are under study. These include:

Paint-on scintillator: PVT base with the dyes butyl-PBD and BBOT. Paint-on waveshifter: PVT base with the dye K27.

Sample of various thicknesses up to $1 / 16$ " have been prepared in our lab and compared to similar thicknesses of standard scintillator such as:

Standard plastic scintillator: PVT with the dyes pTP and bis-MSB.

Standard plastic waveshifter: PVT with the dye K27.

Figure 46 shows the results from the preliminary measurements in various combinations:

1. Standard plastic scintillator and standard plastic waveshifter.

2. Paint-on scintillator and standard plastic waveshifter.

3. Standard plastic scintillator and paint-on waveshifter.

4. Paint-on scintillator and paint-on waveshifter.

Under these tests, the geometric arrangement follows the layouts in Figures 42-44 for bulk solids and liquids testing. Sample excitation was by ${ }^{90} \mathrm{Sr}$ source and the photosensor was an R1104 PMT. Optical filters were used to remove light from the scintillator as much as possible, and to allow only the light from the waveshifter to pass. Since the emission is broad from these types of materials, several filters were used with successively higher, passing wavelengths to observe trends. Cutoffs were at $475 \mathrm{~nm}$, $495 \mathrm{~nm}$ and $515 \mathrm{~nm}$ for the filters displayed in Figure 46. As can be seen, the standard 
combination \#1 of standard plastic scintillator and waveshifter (upper left-hand in Figure 46) yielded the best efficiency. This is not surprising as these material compositions are optimized. What is remarkable is how well the paint-on materials performed, even though no optimization has been carried out on material composition.

Comparing the observed mean light yield at the longest wavelength filter (Filter \#3) provided best elimination of any non-waveshifted emission from the scintillation material:

Sample \# 2 vs \#1: The efficiency of combination \#2 (liquid scintillator and standard plastic waveshifter) is $85 \%$ of combination \#1, indicating that the painton scintillator is an effective light-emitter - but material or mechanics are not yet optimized.

Samples \#4 vs \#3: Similar performance of either type of scintillator when shifted by paint-on shifter.

Samples \#2 vs \#3: Similar performance of either type of waveshifter in combination with either scintillator.

Samples \#1 vs \#4: Plastic scintillator shifted more effectively with plastic waveshifter than with paint-on waveshifter. Explanation is likely concentration of dye in paint-on vs plastic waveshifter.

More tests need to be run with different thicknesses, curing times, etc. This is a rich area for future $R \& D$ activity. 


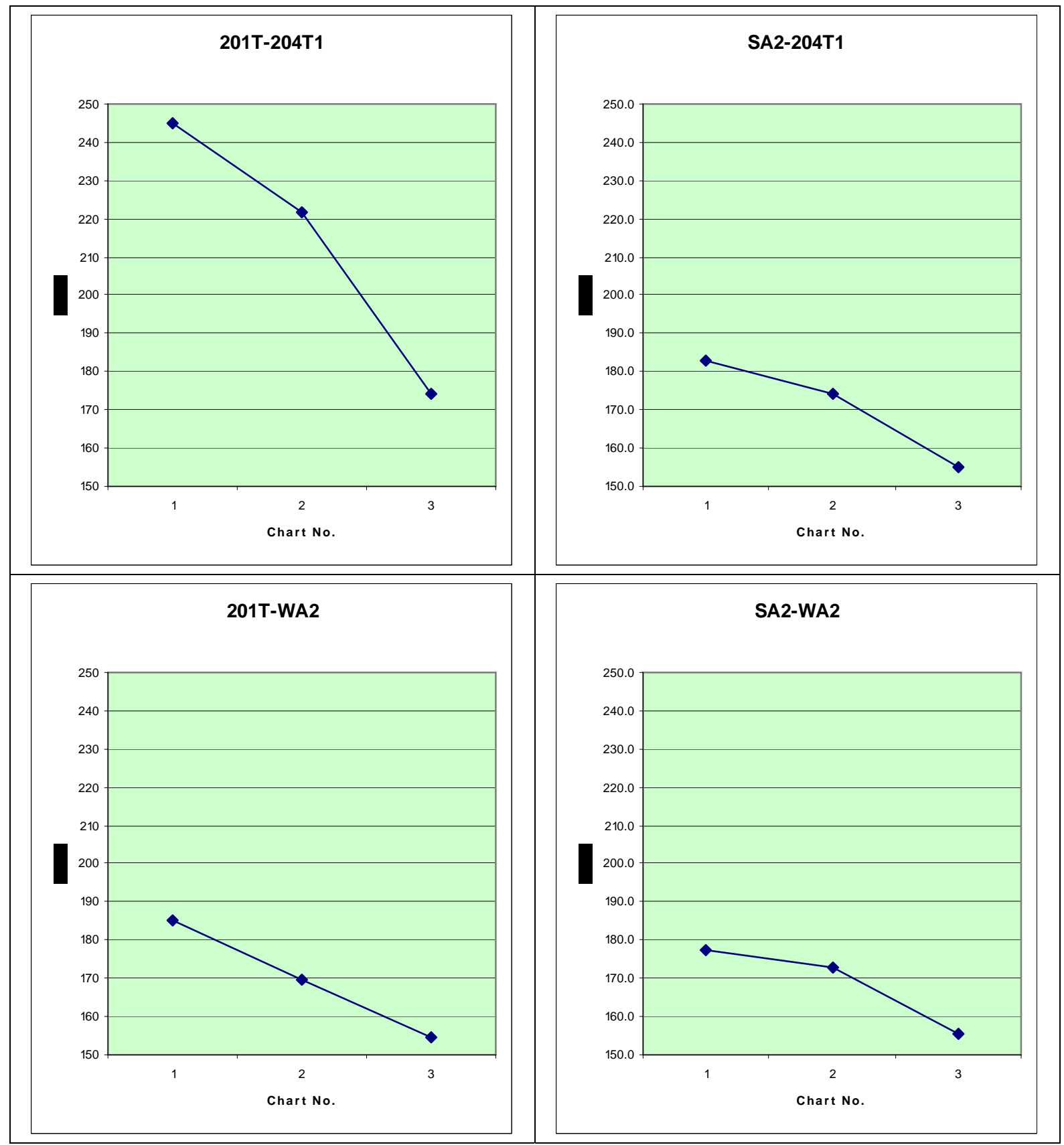

Figure 46. Mean pulse height (arbitrary units) observed for scintillator/waveshifter combinations as a function of optical filter. Samples were excited with a ${ }^{90} \mathrm{Sr}$ Source. Upper left, standard plastic scintillator and standard waveshifter. Upper right, paint-on scintillator and standard waveshifter. Lower left, standard plastic scintillator and paint-on waveshifter. Lower right, paint-on scintillator and paint-on waveshifter. Scintillator and waveshifter thicknesses were $\sim 1 / 16$ " thick in all cases. Filter \#1 had transmission above 475nm; Filter \#2 had transmission above 495nm; Filter \#3 had transmission above 515nm. 


\section{Section 7. Comments on Educational and Workforce Development}

This research program included significant effort in the area of workforce development. In addition to the assigned team of physicists/engineers/technicians, we have also had high school teachers and students working with us over several years. These individuals have worked as team members in all areas, including: materials preparation and detector setup, data recording, and analysis.

Over the course of this project, 3 high school teachers and 16 high school students have participated in scintillator and waveshifter research.

Several of the participating high school students entered their research into the National Siemens Competition. In Fall 2006, two of these students, reached the Semifinals of the competition and were selected to move on to the Siemens Regionals and present their work at MIT. Both of these young women, shown in Figure 47, are now at MIT as undergraduates. In Fall 2007, three other students from the group reached the Semifinals but were not selected to move on to the Regionals. This was a very high level of achievement for these young people, and a strong recognition of the importance of this Ludlum Sub-award to Notre Dame for its ability to attract nontraditional participants into the research process.

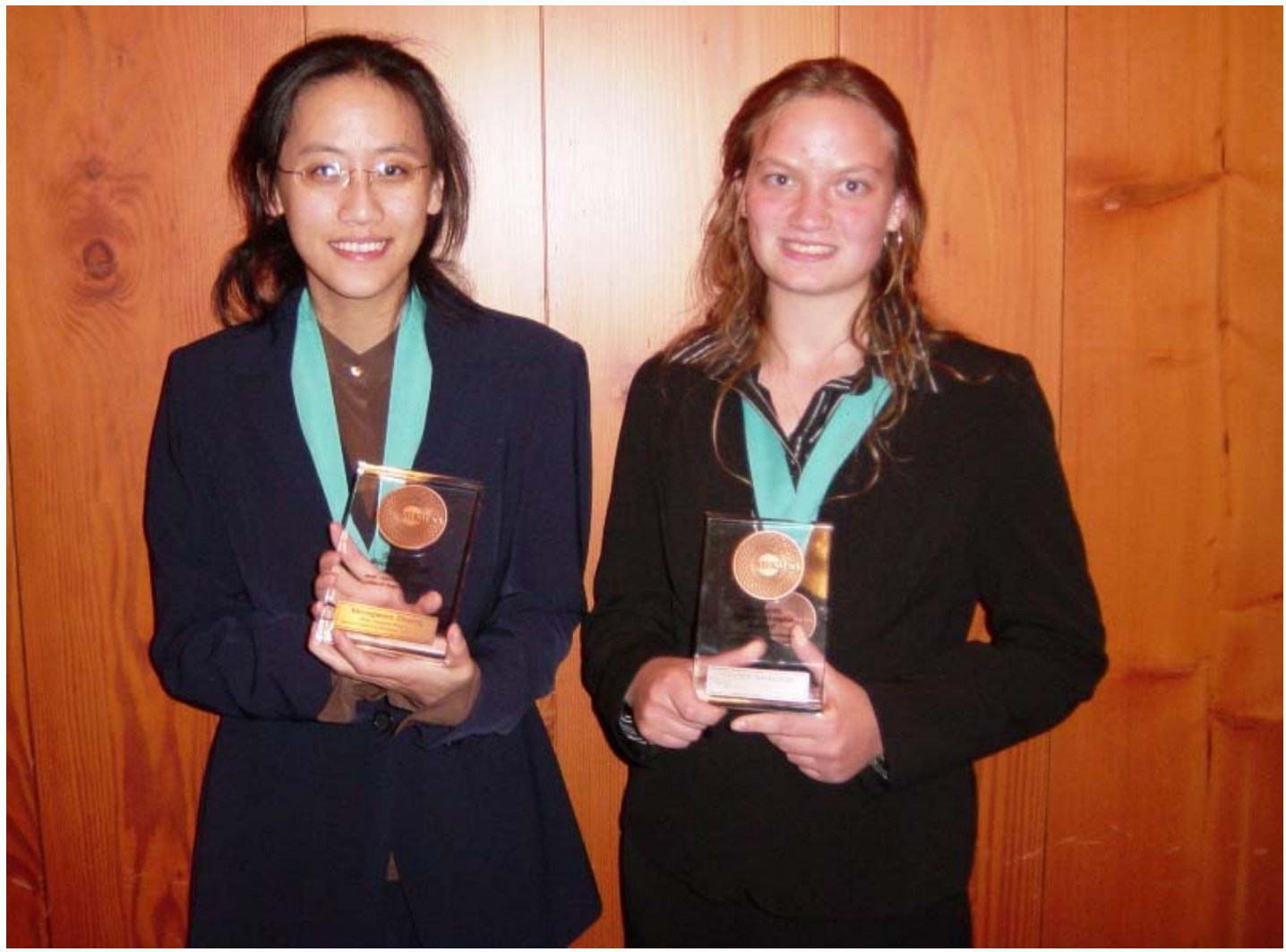

Figure 47. Mengwen Zhang and Kristen Anderson were named Regional Finalists in the 2006-2007 Siemens Competition. Their research, entitled "Performance of Scintillating and Waveshifting Fibers for Particle Detectors," was done at the University of Notre Dame's QuarkNet Center. 


\section{Section 8. Conclusions}

This has been a very successful program.

Over a half-dozen new dyes have been developed and tested as potential replacements for Y11. The results of these tests in bulk and in tile/fiber geometry indicate that they offer superior performance. Future tests of radiation damage will need to be carried out to assess the full range of applicability of these materials.

New liquid scintillator compositions have been developed whose performance exceeds that of standard liquid cocktails built around BBOT or BBQ. And paint-on materials for potential use in thin coating applications have been identified and are under development.

Alternative materials as potential replacements for $3 \mathrm{HF}$ have been identified, although none of the materials offers the same fluorescence efficiency as 3HF - at least, in the concentrations studied and as long as the use of the fiber material is handled in a very controlled (light-controlled) environment.

More targeted work can be directed in a number of areas, and should be followed up rapidly, to take advantage of LHC and ILC detector development in particle physics and for new detector concepts for neutrino physics and physics at the Deep Underground Science and Engineering Laboratory (DUSEL) project, under advancement.

Potential Impact Areas (in High Energy Physics)

1. SLHC

i. $\quad$ Calorimetry upgrades

ii. Muon triggering

iii. SiPM R\&D

2. ILC

i. Calorimetry

ii. Muon Detectors

iii. Tracking \& Triggering

iv. SiPM R\&D

3. Neutrinos \& DUSEL

i. $\quad$ Tracking \& Triggering

ii. Particle identification 


\section{Section 9. Appendices}

Papers based upon work from the Sub-award. Paper submissions to IEEE Nuclear

Science Symposia in 2003 and 2005 and paper publications in ICATPP-8 and ICATPP-9. 


\title{
Waveshifters and Scintillators for the Detection of Ionizing Radiation
}

\author{
R. Ruchti, D. Karmgard, M. Albrecht, K. Andert, P. Anselmino, B. Baumbaugh, J. Bishop, \\ V. Clendenen, H. Dauerty, D. Dreher, C. Hurlbut, M. Jensen, N. Kamat, B. Marchant, \\ J. Marchant, M. McKenna, A. Rozzi, A. Slusher, R. Sommese, T. Sparks, and M. Vigneault
}

\begin{abstract}
New waveshifter and scintillator materials are under development for use in detecting charged particles in tracking applications and for detecting showering particles in calorimetric applications. Goals have been to identify and produce fast and efficient dye materials that fluoresce in the middle of the visible spectrum where polystyrene and polyvinyltoluene have good optical transparency, to replace existing materials currently in use in the field of particle physics. As a result of this study, several fluorescent dyes have been identified with fast and efficient emission, that fluorescence in the green $(\lambda \sim 490-520 \mathrm{~nm})$, and from these a number waveshifter and scintillator materials have been fabricated.
\end{abstract}

\section{INTRODUCTION}

$\mathrm{O}$ VER the last decade several techniques have emerged for the readout of particle physics detectors for scintillation tracking and sampling calorimetry.[1] We

Manuscript received October 23, 2003. This work was supported in part by the U.S. Department of Energy SBIR and STTR Programs, the U.S. National Science Foundation QuarkNet and RET Programs, and the University of Notre Dame.

R. Ruchti is with the Department of Physics, University of Notre Dame, Notre Dame, IN 46556 USA (telephone: 574-631-4737, email: rruchti@nd.edu)

D. Karmgard is with the Department of Physics, University of Notre Dame, Notre Dame, IN 46556 USA (telephone: 574-631-3362, email: karmgard.1@nd.edu)

M. Albrecht, K. Andert, P. Anselmino, H. Dauerty, D. Dreher, M. Jensen, N. Kamat, B. Marchant, A. Rozzi, A. Slusher, R. Sommese, and T. Sparks are with the University of Notre Dame Department of Physics and QuarkNet Center, Notre Dame, IN 46556 USA (telephone: 574-631-3773, email: quarknet@nd.edu)

B. Baumbaugh is with the Department of Physics, University of Notre Dame, Notre Dame, IN 46556 USA. (telephone: 574-631-3774, email: baumbaugh.1@nd.edu)

J. Bishop was with the University of Notre Dame, Notre Dame, IN 46556. $\mathrm{He}$ is now at $5516 \mathrm{~N}$. Laguna Dr Litchfield Park AZ 85340 (telephone: 623935-7078, email: jmmbishop64@cox.net)

C. Hurlbut and V. Clendenen are at Ludlum Measurements Inc, 501 Oak Street, PO Bo 810, Sweetwater, TX 79556 USA (telephone: 325-236-9468, email: churlbut@eljentechnology.com)

J. Marchant is with the Department of Physics, University of Notre Dame, Notre Dame, IN 46556 USA. (telephone: 574-631-9941, email: marchant.1@nd.edu)

M. McKenna is with the Department of Physics, University of Notre Dame, Notre Dame, IN 46556 USA (telephone: 574-631-3870, email: mckenna.36@nd.edu)

M. Vigneault is with the Department of Physics, University of Notre Dame, Notre Dame, IN 46556 USA (telephone: 574-631-3870, email: vigneault.1@nd.edu). comment briefly on two here. For tracking detectors, of which the most ambitious example is the DØ Central Fiber Tracker [2], each fiber in the tracking detector has its own photosensor. The active detector material is multi-clad polystyrene scintillating fiber of $830 \mu \mathrm{m}$ diameter and in lengths of up to $2.7 \mathrm{~m}$. The scintillator is based on a primary dye p-terphenyl and a secondary dye 3-hydroxyflavone. The scintillating fibers are optically coupled to fiber-optic waveguides of the same diameter and in lengths of over 8 meters which convey the scintillation light to cryogenically cooled photosensors called visible light photon counters (VLPC).

For calorimetric detectors, exemplars are the "megatile" and "tile" sampling calorimeters of CDF [3], CMS [4] and ATLAS [5]. Plates (or "tiles") of conventional blue-violet emitting scintillator are read out with embedded multi-clad waveshifter fiber containing the dye Y11. The base materials of the scintillating tiles are formed of either polystyrene or polyvinyltoluene (PVT) depending upon cost or placement in the experiments. Of the two, PVT-based materials are the most efficient, but PVT also tends to be more expensive and less radiation resistant than polystyrene.

The major research and development effort considered in this paper focuses on advances in calorimetric technique improvement in the waveshifting of blue-violet scintillation materials and in the development of new green-emitting scintillators. Since tile/plate materials can be produced in either polystyrene or PVT, but fiber can only be produced effectively in polystyrene, the research effort has focused on both compositions.

\section{SCINTILLATORS AND WAVESHIFTERS}

Plastic samples of PVT and polystyrene were polymerized in 1-inch diameter test tubes and then sliced and polished into disks of $1 \mathrm{~cm}$ thickness for further study.

Standard samples were fabricated to form a reference base to which other materials could be compared. The chosen scintillation standard was EJ200. This modeled the brightest conventional blue-violet emitting scintillator with $\lambda \sim 425 \mathrm{~nm}$.

To waveshift the light from this standard scintillator, we chose the highly efficient dye Y11 in PVT and in polystyrene. [6] 
Several hundred new samples of various types were prepared (polymerized) similarly in 1-inch test tubes with a variety of new fluorescent dyes or dye combinations. Some of these materials were scintillators - containing primary and secondary dyes. Others were waveshifters only - containing no primaries.

\section{A. Optical Characteristics of Scintillators and Waveshifters}

Initially the materials were characterized as to their optical properties. Emission and excitation spectra for each of the samples were measured with a Hitachi F2000 Spectrophotometer attached to a custom data acquisition system. Figure 1 displays such spectra for the standard scintillator, standard waveshifter (Y11), and two new shifter dyes (DSB1 and DSB2) produced in this program. Once the emission properties were known, fluorescence decay times of the samples were measured with a nitrogen laser spectrometer. Samples were excited at $\lambda=337 \mathrm{~nm}$, and the fluorescence decay at emission maximum observed. The comparative measurements for the Standards and DSB1 and DSB2 are displayed in Figure 2.
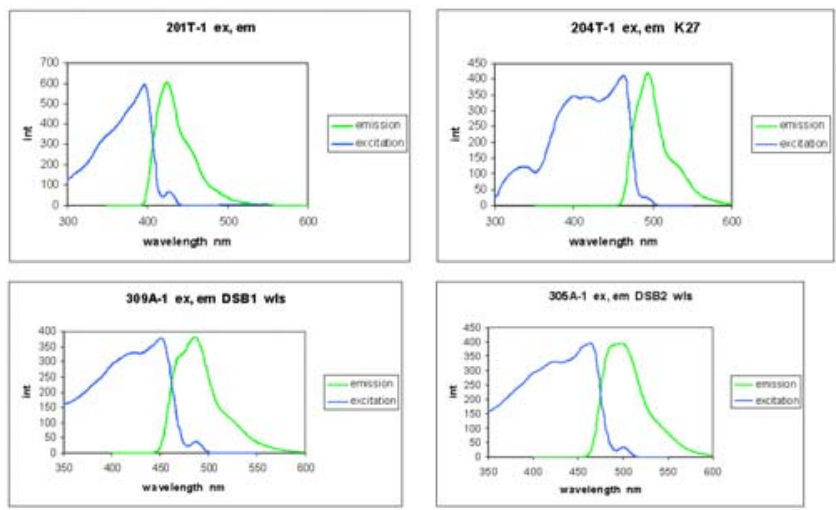

Fig. 1. Emission and absorption spectra. Standard blue-violet emitting scintillator (upper left). Standard Y11 waveshifter (upper right). New DSB1 waveshifter (lower left). New DSB2 waveshifter (lower right). The spectral excitation and emission properties of DSB1 and DSB2 are seen to be similar to Y11.

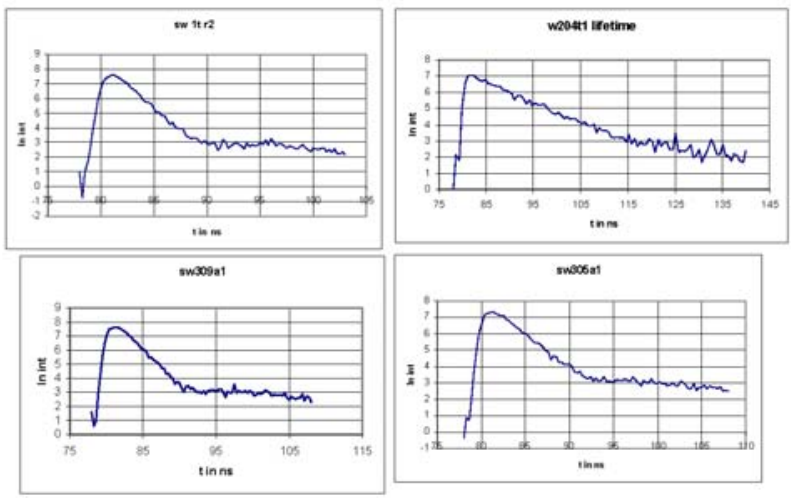

Fig. 2. Fluorescence decay times excited by a nitrogen laser at $337 \mathrm{~nm}$. Light is detected at the emission maximum for samples shown in Figure 1, and fit to a single exponential decay in time after the peak of each pulse. Standard blueviolet emitting scintillator, $1.67 \mathrm{~ns}$ (upper left). Standard Y11 waveshifter, $8.3 \mathrm{~ns}$ (upper right). New DSB1 waveshifter, 1.8ns (lower left). New DSB2 waveshifter, $2.2 \mathrm{~ns}$ (lower right).
The spectral measurements of Figures 1 and 2 reveal that the new dyes are spectrally comparable to the standard waveshifter and as such are an excellent spectral match for the standard scintillator. Additionally they are considerably faster by a factor of 3-4 in fluorescence decay than the standard Y11 waveshifter when excited optically.

\section{B. Scintillator Studies}

Efficiencies of the scintillator samples were characterized by exposing them to a ${ }^{90} \mathrm{Sr}$ beta source. Signals were detected with a Hamamatsu R1104 photomultiplier connected to a LeCroy QVT pulse height analyzer and read into a custom computer data acquisition system. Figure 3 displays the QVT spectra for two samples, the standard scintillator and a new scintillator composition that contains the new dye DSB1 as the secondary. The DSB1 measurement, once compensated for the reduced quantum efficiency of the R1104 PMT at the longer emission wavelength of the material $(\lambda \sim 490 \mathrm{~nm})$, reveals a scintillator with essentially $80 \%$ of the efficiency of the standard. A similar result is found for a new scintillator containing DSB2 with emission $\lambda \sim 500 \mathrm{~nm}$.

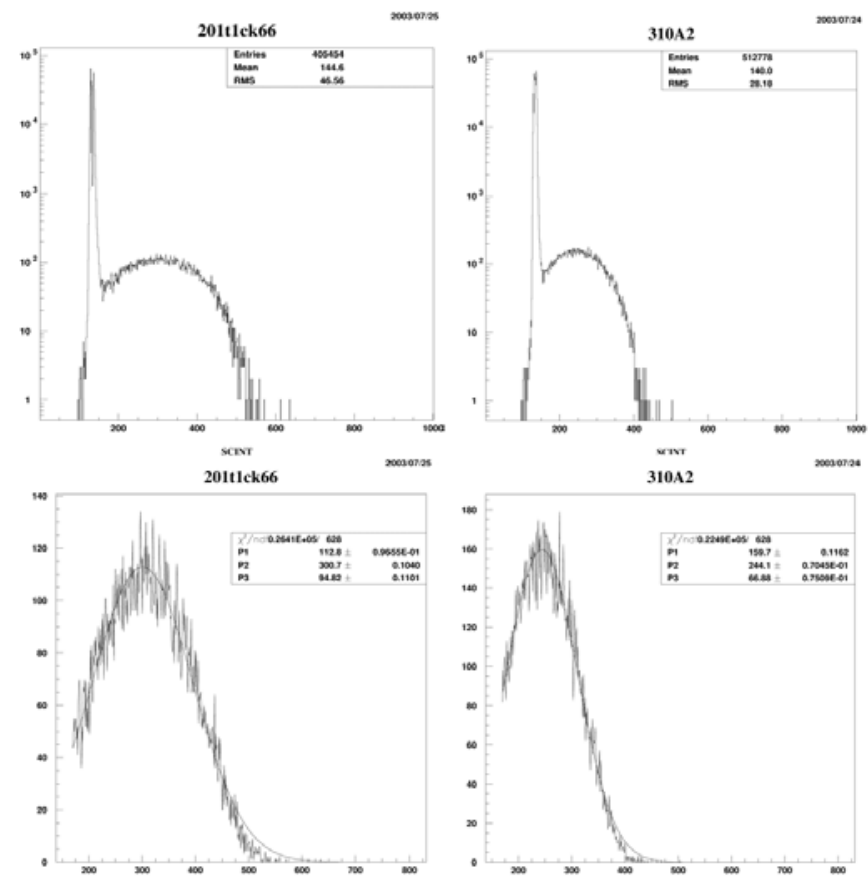

Fig. 3. QVT pulse height spectra for standard blue-violet emitting scintillator upper and lower left and a new scintillator containing a primary and the secondary DSB1 at the upper and lower right. Upper spectra are semi-log plots. Lower spectra are linear plots. Horizontal axes are in least counts of the QVT $(0.25 \mathrm{pC}$ per least count). Pedestals are seen in the upper plots and have been suppressed in the lower plots. Although the DSB1 spectra do not appear to be as strong, these are not yet corrected for the wavelength dependent response of the photocathode of the R1104 PMT used to detect the scintillation light. Once corrected, the performance is much more closely equal, with the DSB1 providing approximately $80 \%$ or more of the response of the standard.

Figure 4 displays the measured scintillation efficiencies as a function of wavelength of a variety of scintillation materials 
prepared in this program. The efficiencies are normalized to the standard scintillator that emits at $\lambda \sim 425 \mathrm{~nm}$, and measurements are corrected (scaled) for the wavelength dependent quantum efficiency of the R1104 PMT. There are a number of interesting features revealed in the figure. For a given type of sample, there is a range of possible efficiencies separated by about $20 \%$ in overall value. Those at the high end are PVT based; those at the low end are polystyrene based. Intermediate values correspond to the choice of primary dyes used in the compositions. The figure reveals a significant number of potentially useful and efficient candidate scintillation materials in the wavelength range $470 \mathrm{~nm} \leq \lambda \leq$ $530 \mathrm{~nm}$.

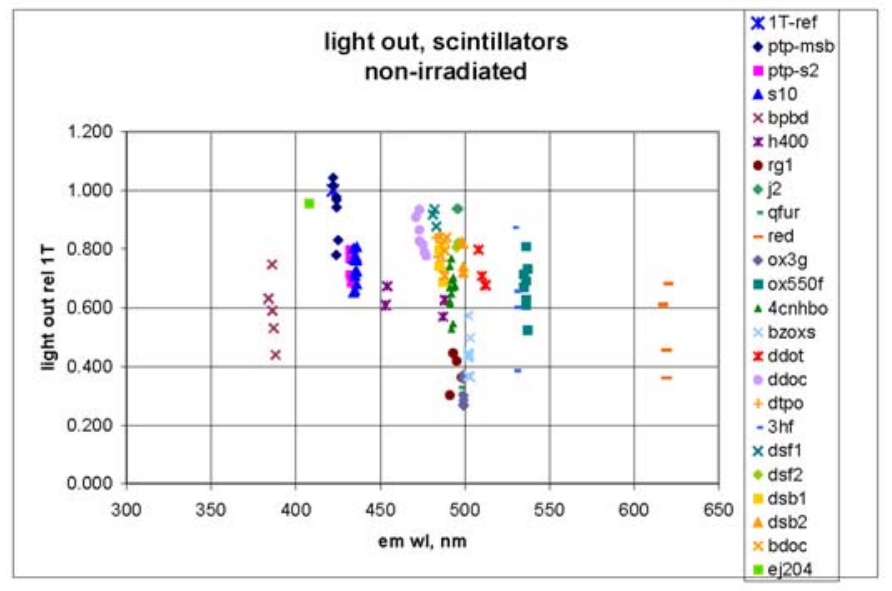

Fig. 4. Scintillation efficiency of a variety of scintillation compositions as a function of peak emission wavelength for each sample. Efficiency is normalized to the scintillation standard at $\lambda=425 \mathrm{~nm}$. Efficiency is measured by means of emission response to a ${ }^{90} \mathrm{Sr}$ beta source. Light is detected with a Hamamatsu R1104 PMT and is corrected (scaled) for wavelength dependent photocathode response of the PMT.

Figure 4 reveals the fluorescence decay time of numerous scintillation samples as a function of wavelength. The fastest of these, with fluorescence near 500nm, are DSF1, DSF2, DSB1, and DSB2.

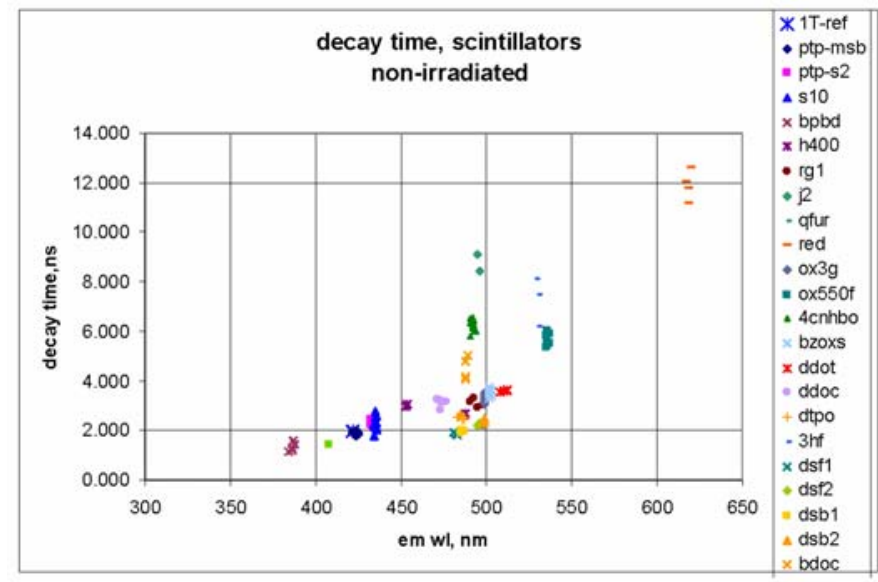

Fig. 5. Fluorescence decay time for a variety of scintillation compositions as a function of peak emission wavelength.

\section{Waveshifter Studies}

Shifter efficiency was determined by exposing the standard scintillator sample to a ${ }^{90} \mathrm{Sr}$ beta source. A waveshifter sample was placed between the scintillator sample and the R1104 PMT. To assure that light from the scintillator did not leak directly through the waveshifter sample into the PMT, a filter foil was inserted between the waveshifter and the PMT to eliminate the scintillator signal from reaching the PMT directly. Figure 6 displays the results of this study as a function of the maximum emission wavelength of the waveshifter. This study provided relatively little definitive information between materials - rather it indicated at the level of the measurements that nearly all the waveshifter samples were fairly comparable in performance in shifting capability in the form of bulk (centimeter thick) samples. Greater discrimination between materials was then provided by fluorescence decay time of the materials as the critical discriminator. These measurements are shown in Figure 7. Measurements were made utilizing the nitrogen laser spectrometer and again reveal the relative speed of DSF1, DSB1, and DSB2 and indicate that K27 (Y11) is slower.

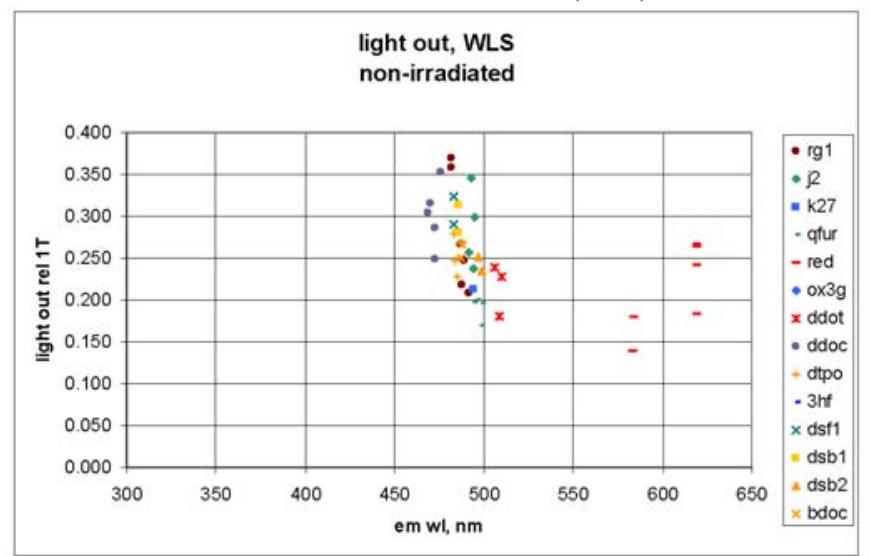

Fig. 6. Waveshifter efficiency as a function of peak emission wavelength of the shifter. Samples are shifting the scintillation light of the standard scintillator excited by a ${ }^{90} \mathrm{Sr}$ beta source. Light is detected with a Hamamatsu R1104 PMT and corrected for wavelength dependent photocathode response.

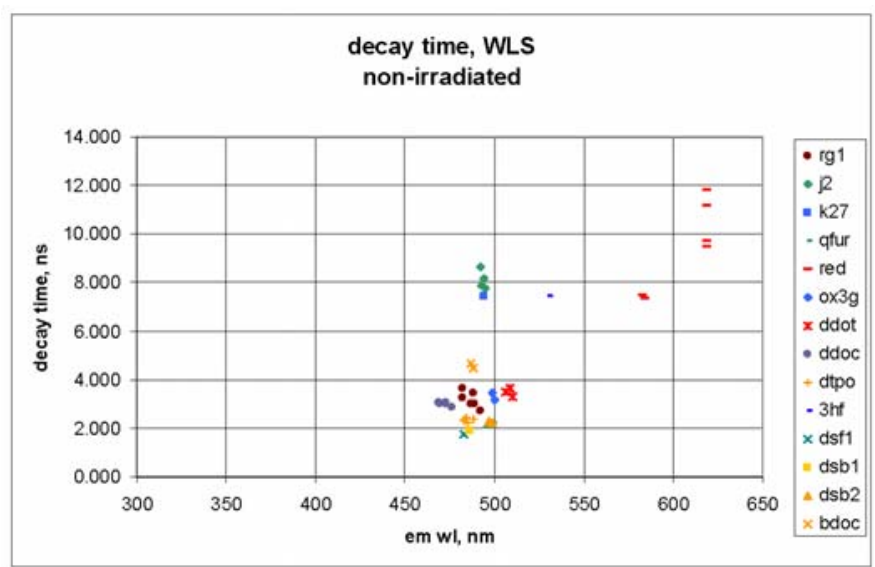

Fig. 7. Fluorescence decay time for a variety of waveshifters as a function of peak emission wavelength. 


\section{TILE-FIBER MEASUREMENTS}

Those materials that revealed promising scintillation performance and rapid fluorescence decay were then incorporated into multi-clad fiber for waveshifter studies. The dye materials so chosen included DSB1, DSB2, BDOC and were compared to the standard dye Y11. These were used to read out scintillation tiles of $10 \mathrm{~cm} \times 10 \mathrm{~cm}$ dimension and $6 \mathrm{~mm}$ thickness of standard scintillation material with emission maximum in the blue-violet. Figure 8 shows the detection system which included a three fold coincidence of trigger counters, including a PMT which viewed the scintillation tile itself and two other thin independent counters one above and one below the tile. The coincidence of these counters was used as a gate for the signal from the waveshifter fiber, which was located in a groove in the tile and read out by means of a Hamamatsu R943 GaAs PMT. The signals from the R943 PMT were averaged for up to 2048 pulses and displayed on an HP54502A digitizing oscilloscope and read into a computer. Such signals could be recorded for cosmic ray triggers and for triggers from a ${ }^{106} \mathrm{Ru}$ beta source. Either generated essentially the same results at our present level of measurement.

Figure 9 displays the averaged pulses for DSB1, DSB2, BDOC and Y11 waveshifters of the standard scintillation tile when excited with the ${ }^{106} \mathrm{Ru}$ source. As can be clearly seen, the DSB1 and DSB2 signals are significantly faster than that of the conventional Y11. The width at half maximum of the DSB1/DSB2 signals is $12.5 \mathrm{~ns}$; for the Y11 signal it is $25 \mathrm{~ns}$. Fitting a single exponential to the fast decay of the DSB1 and DSB2 signals yields decay times of $8 \mathrm{~ns}$ and $9 \mathrm{~ns}$ respectively that includes the time response of the tile, the fluorescent dyes as well as the response characteristics of the R943 PMT. A similar fit to the Y11 signal yields a decay time of $25 \mathrm{~ns}$. Integrating over the full 100ns time window, the fluorescence yield of Y11 and DSB1 are comparable, whereas DSB2 is slightly less efficient $(\sim 5 \%)$. However over shorter time intervals, DSB1 and DSB2 are clearly superior to Y11 in performance.

\section{RAdiation Resistance StUdies}

To date, our radiation resistance measurements have been limited to exposure of small 1-inch diameter x 1-cm thick bulk samples to doses of $1 \mathrm{Mrad}$ of ${ }^{60} \mathrm{Co}$. Measurements of the relative efficiency of samples before and after radiation exposure are presented as a function of sample emission wavelength in Figure 10. When irradiated, samples were maintained in an inert (nitrogen) atmosphere. The figure reveals that typically samples lost approximately $10 \%$ of efficiency after $1 \mathrm{Mrad}$ of exposure.

Clearly a great deal more work needs to be done here. Actual detector elements, for example tile-fiber materials, now need to be exposed to radiation in doses of varying amounts and under various exposure rates, to have a clear understanding of the radiation resistance behavior of these new materials. This is an important topic of future study in the program.

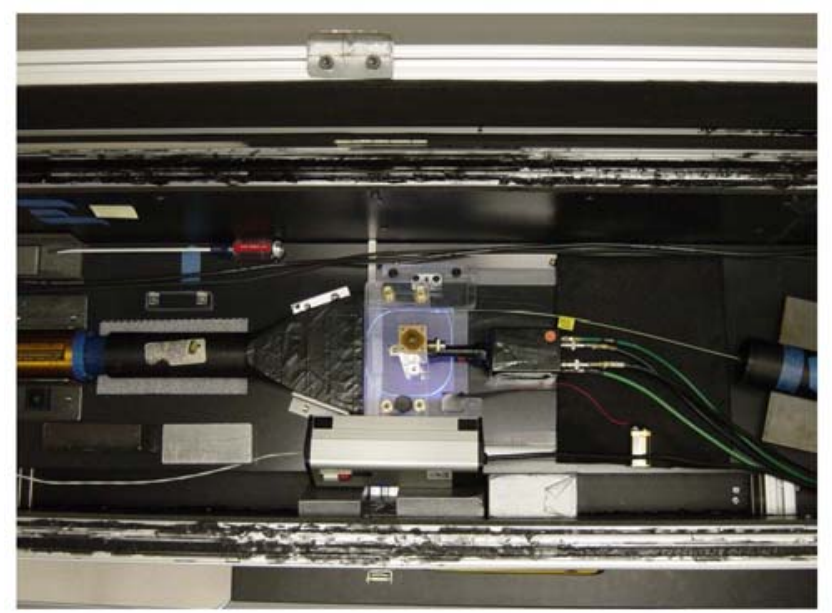

Fig. 8. Detection system for tile-fiber measurements in which fluorescence efficiency of fiber waveshifters is studied. Scintillating tile with embedded wavelength shifter fiber is visible in the center of the figure. The PMT at the right is the R943 PMT that detects the light from the shifter fiber.

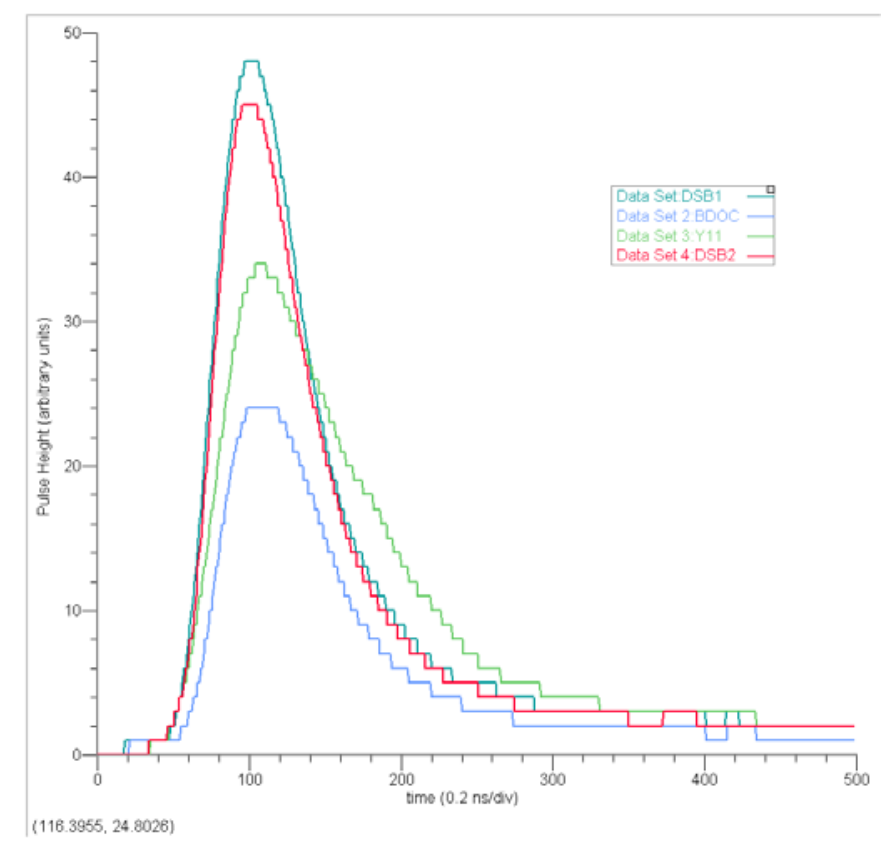

Fig. 9. Averaged pulses recorded for various waveshifter fibers detecting light from a scintillating tile excited by a ${ }^{106} \mathrm{Ru}$ source. The fast pulses are due to DSB1 and DSB2, two new dyes developed in this program. Y11 is the intermediate-sized and slowest pulse. The smaller pulse is due to the new dye BDOC which, although fast, is less efficient at shifting blue-violet light. The main divisions of the horizontal axis in the figure are $20 \mathrm{~ns}$ intervals and the full time interval on the horizontal axis is $100 \mathrm{~ns}$. 


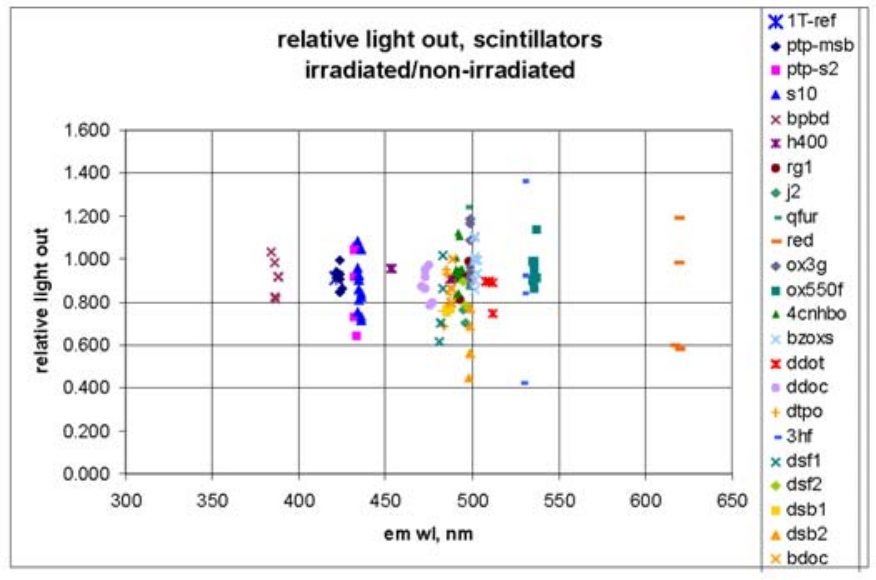

Fig. 10. Measurement of the scintillation efficiency of small 1-inch by 1-cm thick samples of scintillator materials exposed to $1 \mathrm{Mrad}$ of ${ }^{60} \mathrm{Co}$ irradiation. What is plotted is the ratio of light yield after irradiation to before irradiation for samples as a function of their maximum emission wavelength. The results indicate that for these very small samples, recovery to $90 \%$ of original efficiency occurs for essentially all materials. As such these measurements are not particularly informative. More definitive studies are required of actual detector materials (tiles and fibers in needed lengths) in order to draw meaningful conclusions.

\section{CONCLUSIONS}

From the studies carried out in this program, a wide variety of new scintillation and waveshifter materials has been produced and studied with fluorescence emission wavelengths in the middle of the visible spectrum and with rapid fluorescence decay times and reasonable efficiency. Of these, several promising materials have been produced, and of these a number have been incorporated into multi-clad fiber and tested as waveshifters in tile-fiber geometry. Initial measurements indicate that at least two of these, DSB1 and DSB2, offer superior performance in fluorescence decay time to conventional Y11 waveshifter while maintaining high fluorescence efficiency. These materials are therefore potential replacements for Y11 in any application where fast timing is required, or where improved signal to noise would be an advantage by utilizing short integration times.

\section{ACKNOWLEDGMENT}

We would like to thank the staff of the Notre Dame Radiation Laboratory for the use of the nitrogen laser spectrometer, A. Pla-Dalmau of Fermilab for assistance with the sample irradiation studies, E. Skup of Fermilab for tile grooving, and K. Kephart of Fermilab for tile polishing and preparation, and to the University of Notre Dame for support for facilities and resources for high school students and teachers at the Notre Dame QuarkNet Center.

\section{REFERENCES}

[1] SCIFI97 Conference on Scintillating and Fiber Detectors, A. D. Bross, R .C. Ruchti, and M. R. Wayne, Eds, AIP Conference Proceedings 450 (1998).

[2] R. C. Ruchti, The Use of Scintillating Fibers for Charged-Particle Tracking, Annu. Rev. Nucl. Part. Sci. 1996. 46: 281-319.

[3] The CDF II Technical Design Report, Fermilab-Pub-96/390-E.

[4] CMS The Hadron Calorimeter Project Technical Design Report, CERN/LHCC 97-31.

[5] ATLAS Tile Calorimeter Technical Design Report (TDR), CERN/LHC/96-42.

[6] The dye Y11 is variously called K27. 


\title{
Fast, Long-Wavelength Scintillators and Waveshifters
}

\author{
K. Andert, B. Baumbaugh, A. Brothers, A. David, H. Gunther, J. Gurrola, D. Karmgard, T. Madlem, J. Marchant, P. \\ McGough, M. McKenna, R. Ruchti, J. Thompson, and M. Vigneault, Dept. Of Physics and QuarkNet Center, \\ University of Notre Dame, Notre Dame, IN 46556 USA
}

L. Hernandez and C. Hurlbut, Ludlum Measurements, Sweetwater, TX 79556, USA

\begin{abstract}
Studies are presented of new blue-green to red emitting scintillator and waveshifter materials for tracking and calorimetry applications for the detection of ionizing radiation. Materials include plastic scintillators, liquid scintillators, and plastic scintillating and waveshifting fibers. Program goals are to develop faster and more efficient detection media for a variety of experimental applications.
\end{abstract}

\section{INTRODUCTION}

$\mathrm{T}$ HIS paper focuses on the development of scintillating and waveshifting materials for calorimetry and tracking applications. Ultimate objectives are to identify and produce materials with high quantum efficiency, fast fluorescence decay and that afford tolerance to radiation exposure.

For calorimetric detectors, a common detector technique is the scintillation sampling calorimeter., ${ }^{1,2,3}$ Plates or "tiles" of blue-violet emitting scintillator are read out with embedded multi-clad waveshifting fiber containing the dye Y11. Light emission is near $\lambda=510 \mathrm{~nm}$ and the fluorescence decay time is relatively long $>7 \mathrm{~ns}$. This paper provides preliminary measurements of scintillating organic plastic and liquid materials containing new fluorescent dyes that are potential replacements for conventional scintillator and waveshifter. In a high radiation environment, scintillator-based liquids may be flushed from a system and replaced by new material as needed. Additionally several of these dyes have been incorporated as waveshifters in multiclad fiber for the readout of tile scintillators.

\section{SCINTILLATORS AND WAVELENGTH SHIFTERS}

Bulk plastic scintillator and waveshifter samples were polymerized in either polystyrene or polyvinyltoluene. Liquid samples were prepared from aromatic, organic liquid-

This work is supported in part by the U. S. Department of Energy SBIR and STTR Programs, the U. S. National Science Foundation and Department of Energy QuarkNet Program, the National Science Foundation RET Program and the University of Notre Dame.

D. Karmgard is with the University of Notre Dame, Notre Dame, IN 46556 USA. (telephone: 574-631-3362, e-mail: karmgard.1@nd.edu).

J. Marchant, is with the University of Notre Dame, Notre Dame, IN 46556 USA. (telephone: 574-631-9941, e-mail: marchant.1@nd.edu).

R. Ruchti is with the National Science Foundation, Arlington, VA 22230 USA (telephone: 703-292-7392, e-mail: rruchti@nsf.gov).

C. Hurlbut is with the Ludlum Measurements, Sweetwater, TX 79556 USA, (telephone: 325-235-4276, e-mail: churlbut@eljentechnology.com). scintillator base. Fiber waveshifters were produced with double-cladding.

\section{A. Optical Characteristics of Scintillators and Waveshifters}

Initially the materials were characterized optically. Emission and excitation spectra for each of the samples were measured with a Varian Cary Eclipse Fluorescence Spectrophotometer attached to a custom data acquisition system. Fig. 1 displays a sample of emission spectra for three new shifter dyes, DSF1 and AH328B in organic liquid base and DSF2 in a polyvinyltoluene base.

The fluorescence decay times of several samples were measured with a Horiba Jobin Yvon Pulsed Diode Laser Excitation System with TBX-04 Picosecond Photo Detection Module. Samples were excited at $\lambda=377 \mathrm{~nm}$ or $\lambda=457 \mathrm{~nm}$ and the fluorescence decay observed at emission maximum. As can be seen in Fig. 2, the photo-excited fluorescence decay time of DSF1 $(\tau=1.3 \mathrm{~ns})$ is significantly faster than standard Y11 ( $\tau=7.4 \mathrm{~ns})$ by a factor of over 5. The decay of AH238B is found to be slower $(\tau=11.5 \mathrm{~ns})$, but the emission wavelength for this dye is considerably longer (in the yellow/red).

\section{B. Scintillation Efficiency Measurements}

Efficiencies of the scintillator samples were characterized by exposing them to a ${ }^{90} \mathrm{Sr}$ beta source. Signals were detected with a Hamamatsu R1104 photo multiplier connected to a LeCroy QVT pulse height analyzer and read into a custom computer data acquisition system. Optical filtering was used between the samples and the phototube to cut out emission from the bulk material and other primary dyes (if used). ${ }^{4}$ Table 1 displays the measured scintillation efficiencies as a function of wavelength for several scintillation materials prepared for this study. The efficiencies are corrected (scaled) for the wavelength dependent quantum efficiency of the R1104 PMT. 

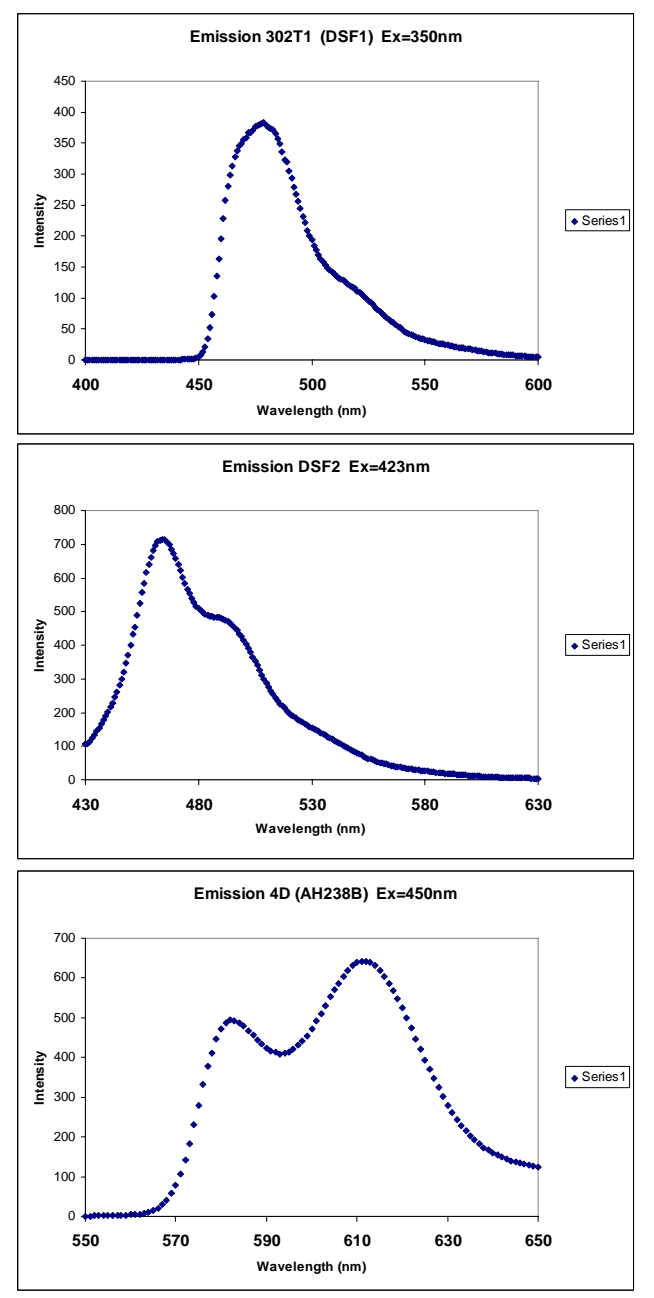

Fig. 1. Emission spectra for several waveshifters. DSF1 waveshifter organic liquid base (top). DSF2 waveshifter in PVT base (middle), AH238B waveshifter in organic liquid base (bottom).

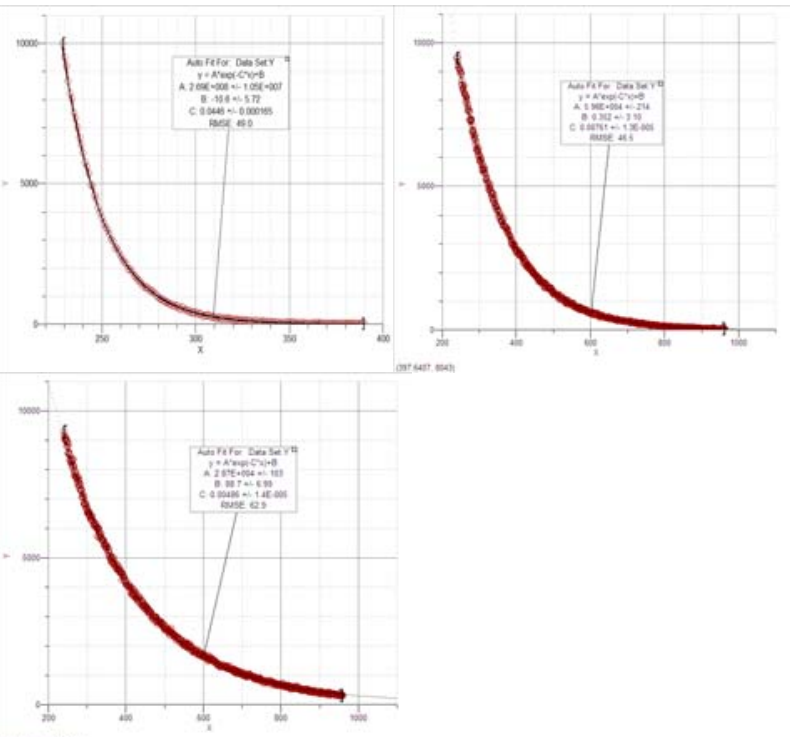

Fig. 2. Fluorescence decay times excited by a Diode Laser at $377 \mathrm{~nm}$. Light is detected at the emission maximum for the samples. DSF1 in organic liquid (upper left); Y11 in PVT (upper right); AH238B in organic liquid (lower left).
TABLE I

SCINTILLATION EFFICIENCY OF SAMPLES

\begin{tabular}{|c|c|c|c|c|}
\hline Composition & $\begin{array}{l}\text { Primary } \\
\text { Dye }\end{array}$ & $\begin{array}{l}\text { Secondary } \\
\text { Dye }\end{array}$ & $\begin{array}{r}\text { Emission } \\
\text { Wave- } \\
\text { length }(\mathrm{nm})\end{array}$ & $\begin{array}{r}\text { Efficiency } \\
\text { Relative to } \\
\text { BBQ }\end{array}$ \\
\hline Liquid L1 & $\mathrm{AH} 238 \mathrm{~B}$ & & 600 & 0.84 \\
\hline Liquid L1 & $\mathrm{P} 1$ & & 450 & 1.05 \\
\hline Liquid L1 & $\mathrm{P} 1$ & $\mathrm{AH} 238 \mathrm{~B}$ & 600 & 1.42 \\
\hline Liquid L1 & P2 & BBQ & 470 & 1 \\
\hline Liquid L1 & P3 & DSF1 & 480 & 1.02 \\
\hline Liquid L1 & P3 & DSB1 & 500 & 1.11 \\
\hline Liquid L1 & P3 & DSB2 & 510 & 1.25 \\
\hline Liquid L2 & P3 & DSF1 & 480 & 1.08 \\
\hline Liquid L2 & P3 & DSF2 & 480 & 1.02 \\
\hline
\end{tabular}

\section{TILE-Fiber MEASUREMENTS}

Those secondary dyes that revealed promising scintillation performance and rapid fluorescence decay were then incorporated into double-clad fiber for waveshifter studies. The dye materials so chosen included DSF1, DSF2, DSB1 and DSB2 and these were compared to the standard dye Y11. All were used to read out scintillation tiles of $10 \mathrm{~cm} \times 10 \mathrm{~cm}$ dimension and $6 \mathrm{~mm}$ thickness of scintillation material (EJ200) with emission maximum in the blue-violet $(\lambda=425 \mathrm{~nm})$. The detection system has been described elsewhere ${ }^{4}$ and includes a three-fold coincidence of trigger counters, including a PMT that views the scintillation tile itself and two other thin independent counters one above and one below the tile. The coincidence of these counters was used as a gate for the signal from the waveshifter fiber, located in a groove in the tile. The optical signal from the fiber was read out using a Hamamatsu H7422P-40 GaAsP PMT. The signals from the PMT were averaged over 2048 pulses and displayed and recorded on an HP54502A digitizing oscilloscope and read into a computer. Such signals were recorded for triggers that occurred in response to a ${ }^{90} \mathrm{Sr}$ beta source.

Fig. 3 displays the recorded signals from the tile/fiber combinations. As can be seen, the best performance is observed for DSB1 shifter and it is superior to Y11 in both overall signal as well as signal response time. DSF1 and DSF2 have lower overall signal, but have very fast response. The data show that for DSF1, the full width at half maximum (FWHM) is 9.0ns and a fit to the tail with a single exponential function reveals a decay time of $\tau=6.1 \mathrm{~ns}$. The values of these quantities for Y11 are FWHM $=17 \mathrm{~ns}$ and $\tau=12.1 \mathrm{~ns}$, indicating the superiority of the DSF1 in time response. Table 2 summarizes the results of these measurements and others. 

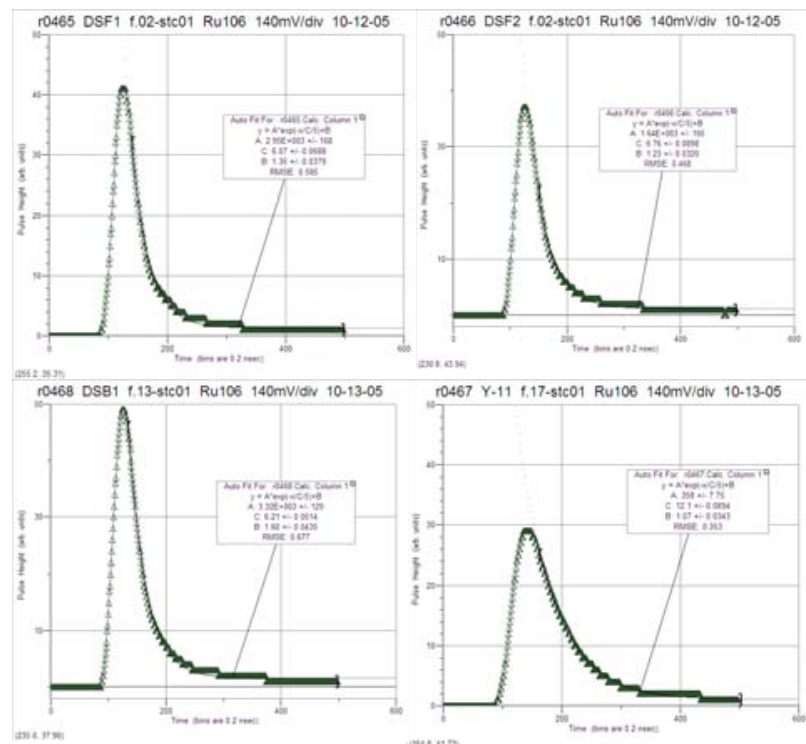

Fig. 3. Averaged output pulses from various waveshifters in double-clad fiber of $940 \mathrm{~mm}$ diameter and $1 \mathrm{~m}$ length reading out EJ200 scintillating tile of $6 \mathrm{~mm}$ thickness and $10 \mathrm{~cm} \times 10 \mathrm{~cm}$ area. Photosensor is a GaAsP PMT. DSF1 (upper left); DSF2 (upper right); DSB1 (lower left); Y11 in PVT (upper right).

$$
\text { TABLE II }
$$

SUMMARY OF WAVESHIFTING EFFICIENCY FOR SAMPLE MATERIALS

\begin{tabular}{llllll} 
Waveshifter & $\begin{array}{l}\text { Emission } \\
\text { wavelength }\end{array}$ & $\begin{array}{l}\text { Efficiency } \\
\text { rel .to Y11 }\end{array}$ & $\begin{array}{l}\text { Decay } \\
\text { Time } \\
\text { Source } \\
\text { Exc. }\end{array}$ & $\begin{array}{l}\text { FWHM } \\
\text { Source } \\
\text { Exc. }\end{array}$ & $\begin{array}{l}\text { Decay } \\
\text { Time } \\
\text { Laser } \\
\text { Exc. }\end{array}$ \\
\hline Y11 & $529 \mathrm{~nm}$ & 1 & $12.1 \mathrm{~ns}$ & $17 \mathrm{~ns}$ & $7.4 \mathrm{~ns}$ \\
DSB1 & $500 \mathrm{~nm}$ & 1.02 & $6.2 \mathrm{~ns}$ & $9.4 \mathrm{~ns}$ & \\
DSF1 & $480 \mathrm{~nm}$ & 0.84 & $6.1 \mathrm{~ns}$ & $9.0 \mathrm{~ns}$ & $1.3 \mathrm{~ns}$ \\
DSF2 & $480 \mathrm{~nm}$ & 0.79 & $6.6 \mathrm{~ns}$ & $9.6 \mathrm{~ns}$ & \\
\hline
\end{tabular}

\section{CONCLUSIONS}

From the studies underway in this program, several new scintillation and waveshifter materials have been produced and studied with fluorescence emission wavelengths in the red to green and with rapid fluorescence decay times and reasonable efficiency. These have been incorporated into multi-clad fiber and tested as waveshifters in tile-fiber geometry (DSF1, DSF2, DSB1, DSB2). Additionally, several of these have been used as secondaries in liquid scintillation cocktails (DSF1, DSF2, DSB1, DSB2, and AH238B). Initial measurements indicate that DSB1, DSF1 and DSF2 offer superior performance in fluorescence decay time to conventional Y11 waveshifter. DSB1 and DSF1 offer comparable integral fluorescence efficiency. These materials are therefore potential replacements for Y11 in any application where fast timing is required, or where improved signal to noise would be an advantage by utilizing short integration times.

\section{ACKNOWLEDGMENT}

We would like to thank P. Kamat and the staff of the Notre Dame Radiation Laboratory for the use of the Diode laser spectrometer, E. Hahn of Fermilab for fiber end-polishing, and to the University of Notre Dame for support facilities and resources for high school students and teachers at the Notre Dame QuarkNet Center.

\section{REFERENCES}

[1] The CDF II Technical Design Report, Fermilab-Pub-96/390-E.

[2] CMS The Hadron Calorimeter Project Technical Design Report, CERN/LHCC 97-31.

[3] ATLAS Tile Calorimeter Technical Design Report (TDR), CERN/LHC/96-42.

[4] M. Albrecht, et al, Scintillators and Wavelength Shifters for the Detection of Ionizing Radiation, Astroparticle, Particle and Space Physics, Detectors ad Medical Physics Applications, ICATPP-8, M. Barone, E. Borchi, C. Leroy, P.-G. Rancoita, P.-L. Riboni, R. Ruchti, eds, World Scientific, 502-511 (2004). 


\title{
SCINTILLATORS AND WAVELENGTH SHIFTERS FOR THE DETECTION OF IONIZING RADIATION*
}

\author{
K. ANDERT, B. BAUMBAUGH, D. KARMGARD, J. MARCHANT, M. MCKENNA, \\ R. RUCHTI, \\ A. SLUSHER, R. SOMMESE, T. SPARKS, AND M. VIGNEAULT ${ }^{\dagger}$ \\ Department of Physics and QuarkNet Center, University of Notre Dame \\ Notre Dame, Indiana 46556, USA \\ V. CLENDENEN AND C. HURLBUT \\ Ludlum Measurements Inc, 501 Oak Street \\ Sweetwater, Texas 79556, USA
}

\begin{abstract}
New scintillators and waveshifter materials are under development for use in detecting charged particles in tracking applications and for detecting showering particles in calorimetric applications. Goals have been to identify and produce fast and efficient dye materials that fluoresce in the middle of the visible spectrum where polystyrene and polyvinyl toluene have good optical transparency, to replace existing materials currently in use in the field of particle physics. As a result of this study, several fluorescent dyes have been identified with fast and efficient emission, that fluorescence in the green $(\lambda \sim$ $490-520 \mathrm{~nm}$ ). These are candidate materials for new scintillators and waveshifters.
\end{abstract}

\section{Introduction}

Over the last decade several techniques have emerged for the readout of particle physics detectors for scintillation tracking and sampling calorimetry. ${ }^{1,2}$ For tracking detectors, of which the most ambitious example is the DØ Central Fiber Tracker, $^{3}$ each fiber in the tracking detector has its own photosensor. The active detector material is multi-clad polystyrene scintillating fiber of $830 \mu \mathrm{m}$ diameter and in lengths of up to $2.7 \mathrm{~m}$. The scintillator is based on a primary dye pterphenyl and a secondary dye 3-hydroxyflavone. The scintillating fibers are optically coupled to fiber-optic waveguides of the same diameter and in lengths of over 8 meters which convey the scintillation light to cryogenically cooled photosensors called visible light photon counters (VLPC).

For calorimetric detectors, examples are the "megatile" and "tile" sampling calorimeters of $\mathrm{CDF}^{4}, \mathrm{CMS}^{5}$ and ATLAS. ${ }^{6}$ Plates or "tiles" of conventional

\footnotetext{
* This work is supported by the U. S. Department of Energy, SBIR and STTR Programs.

${ }^{\dagger}$ Work is partially supported by the U. S. National Science Foundation QuarkNet and RET Programs and the University of Notre Dame.
} 
blue-violet emitting scintillator are read out with embedded multi-clad waveshifter fiber containing the dye Y11. The base materials of the scintillating tiles are formed of either polystyrene or polyvinyl toluene (PVT) depending upon cost or placement in the experiments. Of the two, PVT-based materials are the most efficient, but PVT also tends to be more expensive and less radiation resistant than polystyrene.

The major research and development effort considered in this paper focuses on advances in calorimetric technique - improvement in the wavelength shifting of blue-violet scintillation materials and in the development of new green emitting scintillators. Since tile/plate materials can be produced in either polystyrene or PVT, but fiber can only be produced effectively in polystyrene, the research effort has focused on both compositions.

\section{Scintillators and Wavelength Shifters}

Plastic samples of PVT and polystyrene were polymerized in 1-inch diameter test tubes and then sliced and polished into disks of $1 \mathrm{~cm}$ thickness for further study. Standard samples were fabricated to form a reference base to which other materials could be compared. The chosen scintillation standard was EJ200. This modeled the brightest conventional blue-violet emitting scintillator with $\lambda \sim 425 \mathrm{~nm}$. To waveshift the light from this standard scintillator, we chose the classic material used by the scientific community, the highly efficient dye Y11 in PVT and or polystyrene. ${ }^{7}$

Several hundred new samples of various types were prepared (polymerized) similarly in 1-inch test tubes with a variety of new fluorescent dyes or dye combinations. Some of these materials were scintillators - containing primary and secondary dyes. Others were waveshifters only - containing no primaries.

\subsection{Optical Characteristics of Scintillators and Waveshifters}

Initially the materials were characterized as to their optical properties. Emission and excitation spectra for each of the samples were measured with a Hitachi F2000 Spectrophotometer attached to a custom data acquisition system. Figure 1 displays such spectra for the standard scintillator, standard waveshifter (Y11), and two new shifter dyes (DSB1 and DSB2) produced in this program. Once the emission properties were known, fluorescence decay times of the samples were measured with a nitrogen laser spectrometer. Samples were excited at $\lambda=$ $337 \mathrm{~nm}$, and the fluorescence decay at emission maximum observed. The comparative measurements for the Standards and DSB1 and DSB2 are displayed in Figure 2.

The spectral measurements of Figures 1 and 2 reveal that the new dyes are spectrally comparable to the standard waveshifter and as such are an excellent 
spectral match for the standard scintillator. Additionally they are considerably faster by a factor of 3-4 in fluorescence decay than the standard Y11 waveshifter when excited optically.
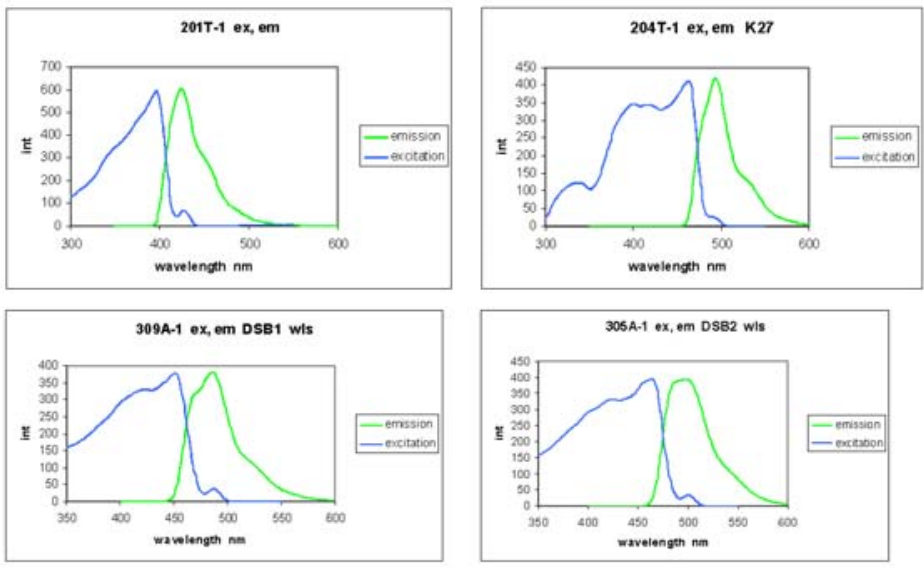

Figure 1. Emission and absorption spectra. Standard blue-violet emitting scintillator (upper left). Standard Y11 waveshifter (upper right). New DSB1 waveshifter (lower left). New DSB2 waveshifter (lower right). The spectral excitation and emission properties of DSB1 and DSB2 are seen to be similar to Y11.

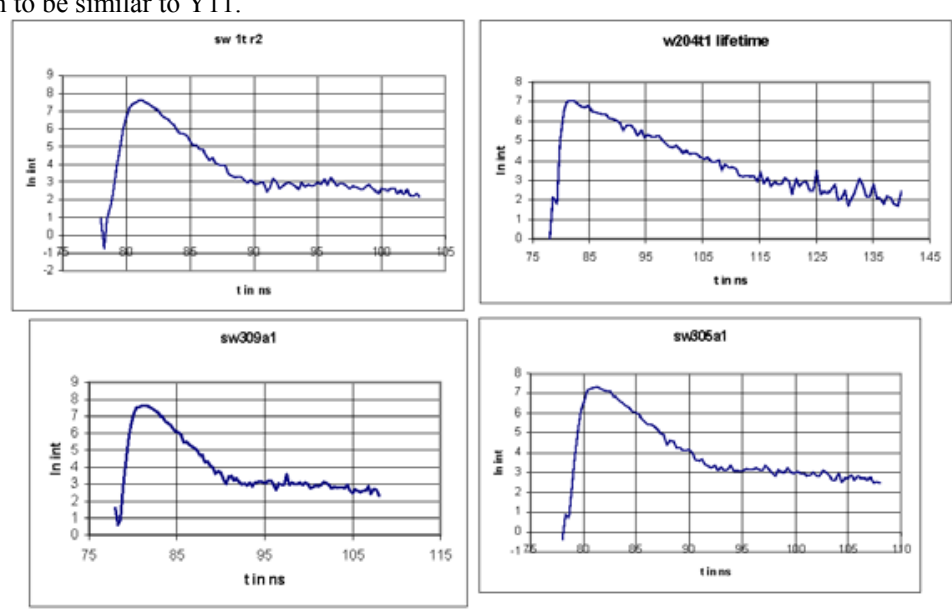

Figure 2. Fluorescence decay times excited by a nitrogen laser at $337 \mathrm{~nm}$. Light is detected at the emission maximum for samples shown in Figure 1, and fit to a single exponential decay in time after the peak of each pulse. Standard blue-violet emitting scintillator, 1.67ns (upper left). Standard Y11 waveshifter $8.3 \mathrm{~ns}$ (upper right). New DSB1 waveshifter, $1.8 \mathrm{~ns}$ (lower left). New DSB2 waveshifter, 2.2ns (lower right). 


\subsection{Scintillator Studies}

Efficiencies of the scintillator samples were characterized by exposing them to a ${ }^{90} \mathrm{Sr}$ beta source. Signals were detected with a Hamamatsu R1104 photo multiplier connected to a LeCroy QVT pulse height analyzer and read into a custom computer data acquisition system. Figure 3 displays the measured scintillation efficiencies as a function of wavelength for a variety of scintillation materials prepared in this program. The efficiencies are normalized to the standard scintillator that emits at $\lambda \sim 425 \mathrm{~nm}$, and measurements are corrected (scaled) for the wavelength dependent quantum efficiency of the R1104 PMT. There are a number of interesting features revealed in the figure. For a given type of sample, there is a range of possible efficiencies separated by about $20 \%$ in overall value. Those at the high end are PVT based; those at the low end are polystyrene based. Intermediate values correspond to the choice of primary dyes used in the compositions. The figure reveals a significant number of potentially useful and efficient candidate scintillation materials in the wavelength range $470 \mathrm{~nm} \leq \lambda \leq 530 \mathrm{~nm}$.

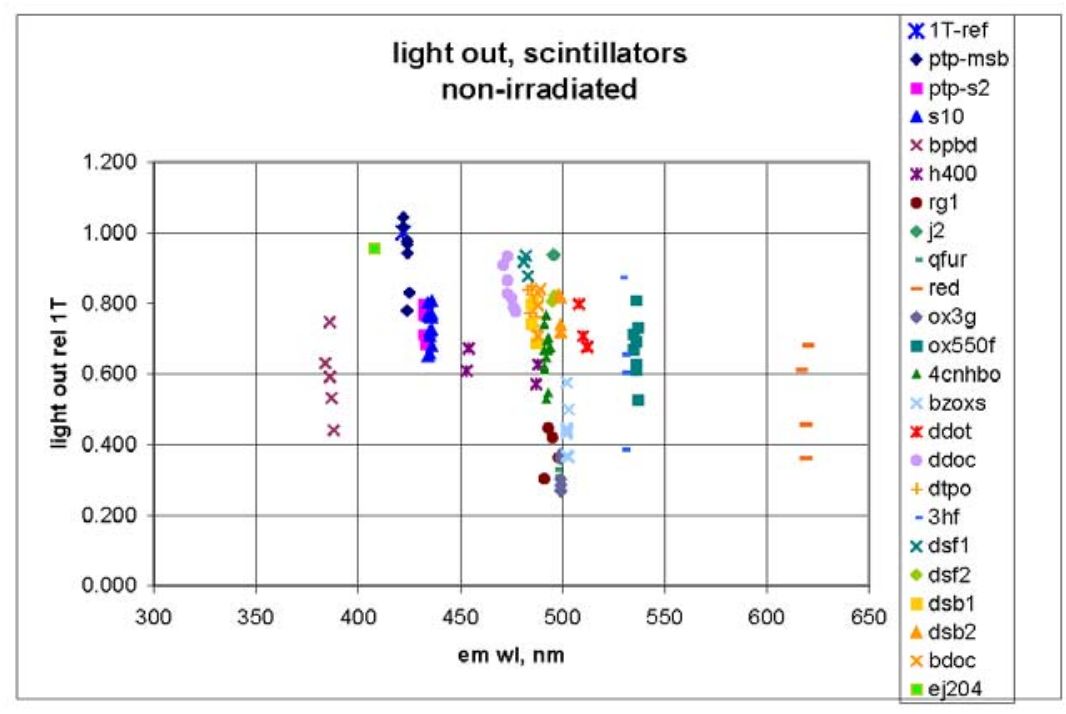

Figure 3. Scintillation efficiency of a variety of scintillation compositions as a function of peak emission wavelength for each sample. Efficiency is normalized to the scintillation standard at $\lambda=425$ $\mathrm{nm}$. Efficiency is measured by response to a ${ }^{90} \mathrm{Sr}$ beta source. Light is detected with a Hamamatsu R1104 PMT and is corrected (scaled) for wavelength dependent photocathode response.

Figure 4 reveals the corresponding fluorescence decay time of the scintillation samples as a function of wavelength measured with the nitrogen 
laser spectrometer. The fastest of these, with fluorescence near 500nm, are DSF1, DSF2, DSB1, and DSB2.

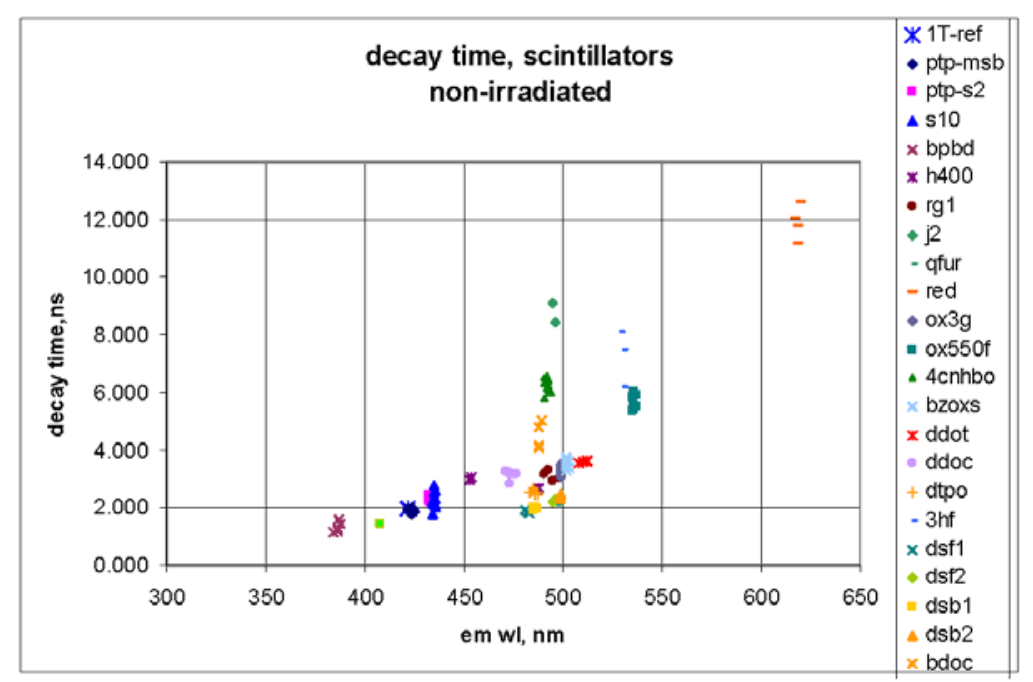

Figure 4. Fluorescence decay time for a variety of scintillation compositions as a function of peak emission wavelength.

\subsection{Waveshifter Studies}

Shifter efficiency was determined by exposing the standard scintillator sample to a ${ }^{90} \mathrm{Sr}$ beta source. A waveshifter sample was placed between the scintillator sample and the R1104 PMT. To assure that light from the scintillator did not leak directly through the waveshifter sample into the PMT, a filter foil was inserted between the waveshifter and the PMT to eliminate the scintillator signal from reaching the PMT directly. Figure 5 displays the results of this study as a function of the maximum emission wavelength of the waveshifter. This study provided relatively little definitive information between materials - rather it indicated at the level of the measurements that nearly all the waveshifter samples were fairly comparable in performance in shifting capability in the form of bulk (centimeter thick) samples. Greater discrimination between materials was then provided by fluorescence decay time of the materials as the critical discriminator. These measurements are shown in Figure 6. Measurements were made utilizing the nitrogen laser spectrometer and again reveal the relative speed of DSF1, DSB1, and DSB2 and similarly indicate that K27 (Y11) is slow. 


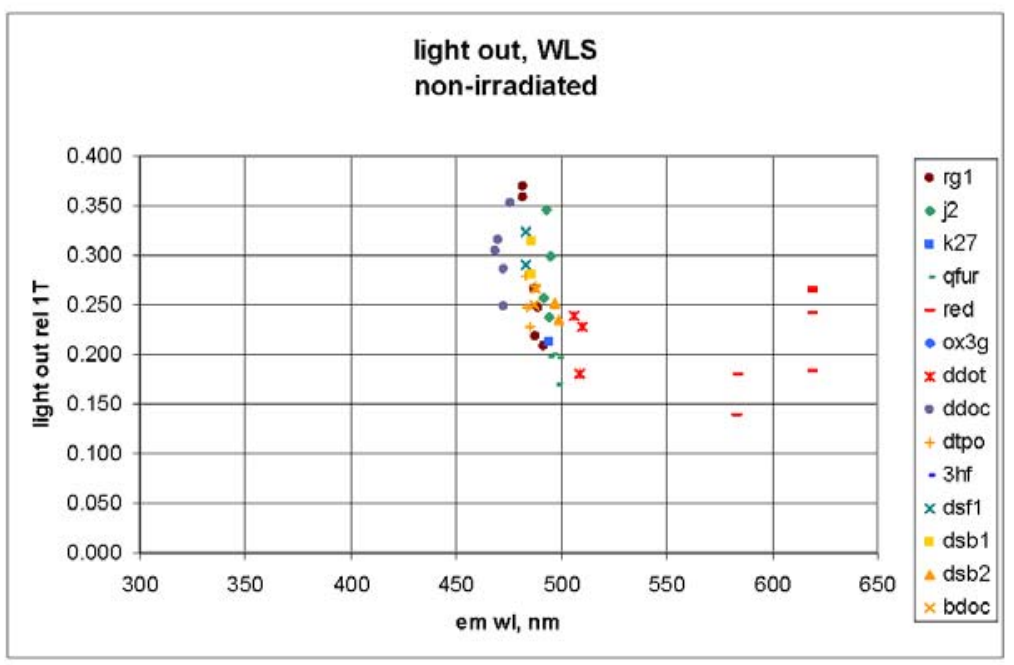

Figure 5. Wavelength shifter efficiency as a function of peak emission wavelength of the shifter. Samples are shifting the scintillation light of the standard scintillator excited by a ${ }^{90} \mathrm{Sr}$ beta source. Light is detected with a Hamamatsu R1104 PMT and corrected for wavelength dependent photocathode response.

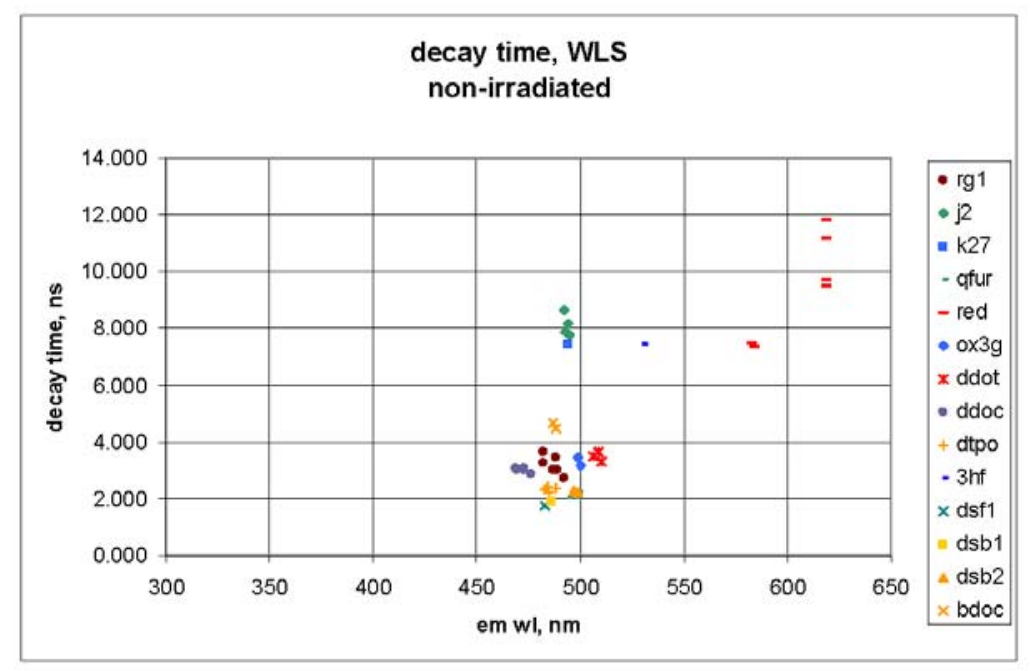

Figure 6. Fluorescence decay time for a variety of wavelength shifters as a function of peak emission wavelength. Samples are excited by a nitrogen laser pulse at $337 \mathrm{~nm}$. 


\section{Tile-Fiber Measurements}

Those materials that revealed promising scintillation performance and rapid fluorescence decay were then incorporated into multi-clad fiber for waveshifter studies. The dye materials so chosen included DSB1, DSB2, BDOC and were compared to the standard dye Y11. These were used to read out scintillation tiles of $10 \mathrm{~cm} \times 10 \mathrm{~cm}$ dimension and $6 \mathrm{~mm}$ thickness of standard scintillation material with emission maximum in the blue-violet. Figure 7 shows the detection system which included a three fold coincidence of trigger counters, including a PMT which viewed the scintillation tile itself and two other thin independent counters one above and one below the tile. The coincidence of these counters was used as a gate for the signal from the waveshifter fiber, which was located in a groove in the tile and read out by means of a Hamamatsu R943 GaAs PMT. The signals from the R943 PMT were averaged for up to 2048 pulses and displayed on an HP54502A digitizing oscilloscope and read into a computer. Such signals could be recorded for cosmic ray triggers and for triggers from a ${ }^{106} \mathrm{Ru}$ beta source. Either generated essentially the same results at our present level of measurement.

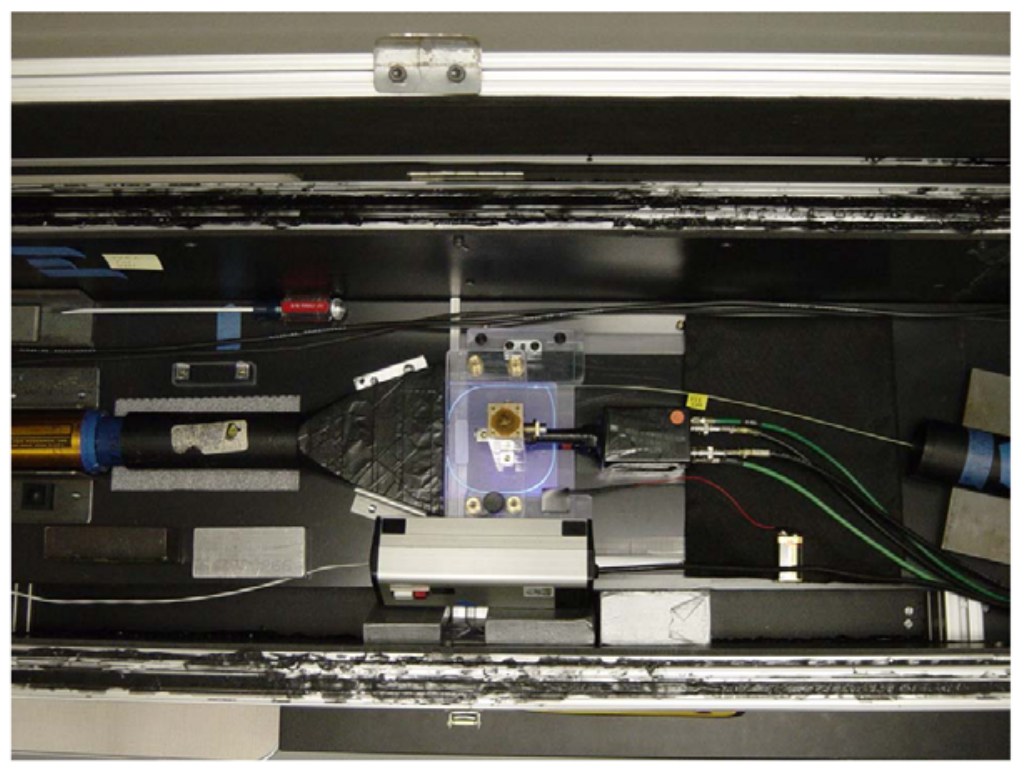

Figure 7. The detection system for tile-fiber measurements in which fluorescence efficiency of fiber waveshifters is studied. The scintillating tile with embedded wavelength shifter fiber is visible in the center of the figure. The black cylinder at the very right center is the R943 PMT that detects the light from the shifter fiber. 
Figure 8 displays the averaged pulses for DSB1, DSB2, BDOC and Y11waveshifters of the standard scintillation tile when excited with the ${ }^{106} \mathrm{Ru}$ source. As can be clearly seen, the DSB1 and DSB2 signals are significantly faster than that of the conventional Y11. The width at half maximum of the DSB1/DSB2 signals is $12.5 \mathrm{~ns}$; for the Y11 signal it is $25 \mathrm{~ns}$. Fitting a single exponential to the fast decay of the DSB1 (DSB2) signal yields a decay time of $8 \mathrm{~ns}$ (9ns) that includes the response of the tile, the fluorescent dyes, as well as the R943 PMT. A similar fit to the Y11 signal yields a decay time of $25 \mathrm{~ns}$. However if one integrates over a full 100ns, the fluorescence yield of Y11 and DSB1 are comparable, whereas DSB2 is slightly less efficient $(\sim 5 \%)$. Over short time intervals DSB1 and DSB2 are clearly superior to Y11 in performance.

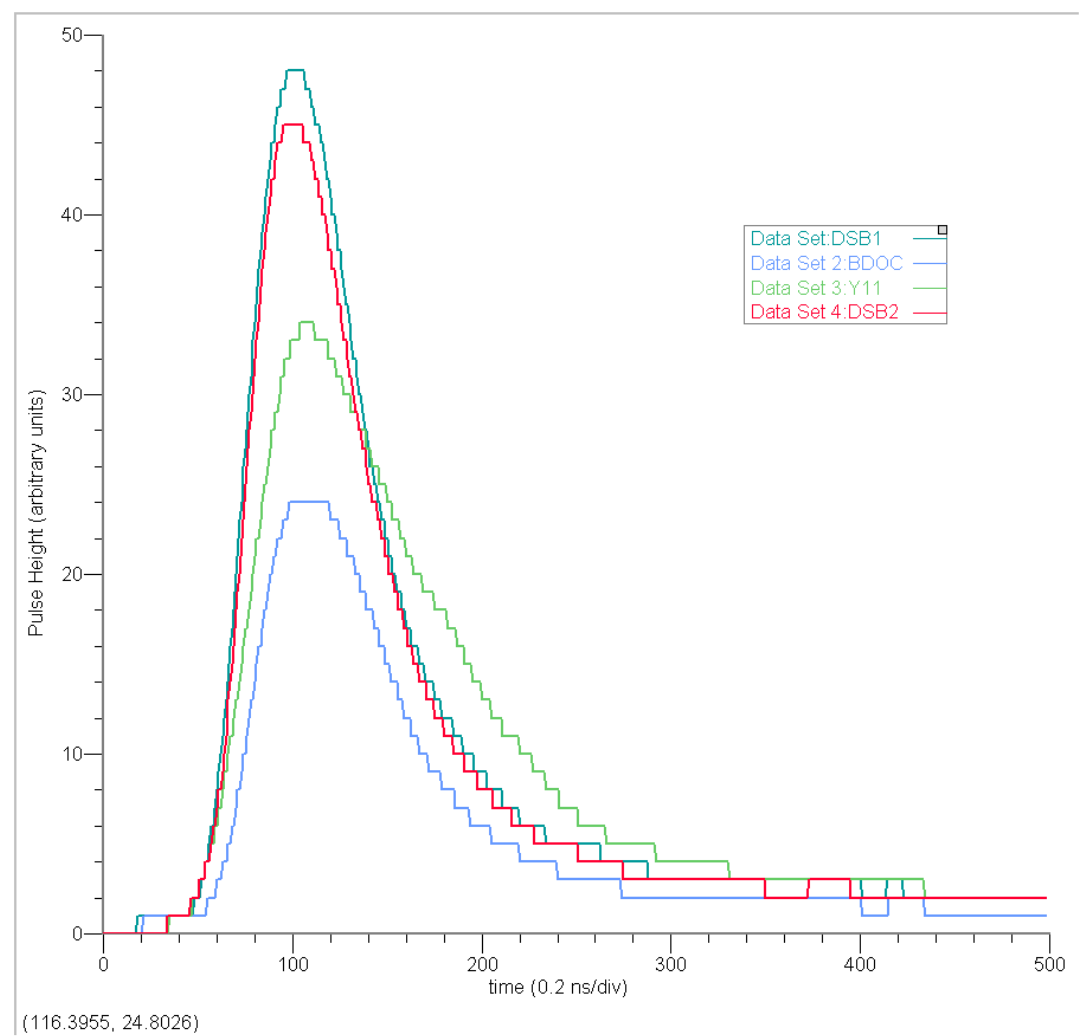

Figure 8. Averaged pulses recorded for various waveshifter fibers detecting light from a scintillating tile excited by a ${ }^{106} \mathrm{Ru}$ source. The fast pulses are due to DSB1 and DSB2, two new dyes developed in this program. Y11 is the intermediate sized and slowest pulse. The smaller pulse is due to the new dye BDOC which, although fast, is less efficient at shifting blue-violet light. The main 
divisions of the horizontal axis in the figure are $20 \mathrm{~ns}$ intervals and the full time on the horizontal axis is $100 \mathrm{~ns}$.

\section{Radiation Resistance Studies}

To date, our radiation resistance measurements have been limited to exposure of the small 1-inch diameter x 1 -cm thick bulk samples to doses of $1 \mathrm{Mrad}$ of ${ }^{60} \mathrm{Co}$. Measurements of the relative efficiency of samples before and after radiation exposure are presented as a function of sample emission wavelength in Figure 9. When irradiated, samples were maintained in an inert (nitrogen) atmosphere. The figure reveals that typically samples lost approximately $10 \%$ of efficiency after 1 Mrad of exposure.

Clearly a great deal more work needs to be done here. Actual detector elements, for example tile-fiber materials, now need to be exposed to radiation in doses of varying amounts and under various exposure rates, to have a clear understanding of the radiation resistance behavior of these new materials. This is an important topic of future study in the program.

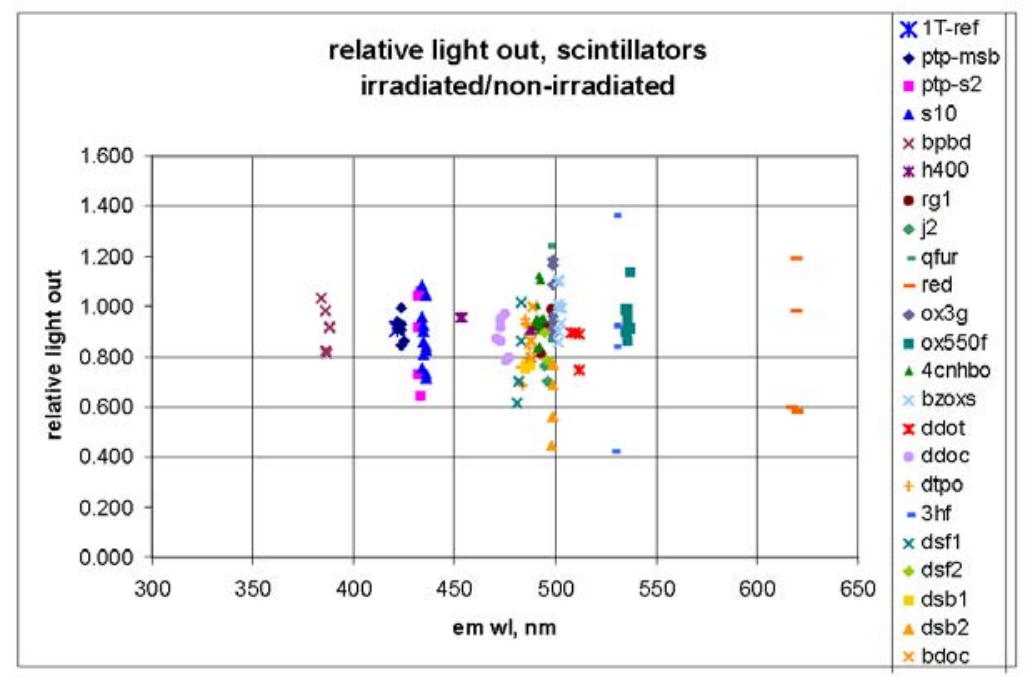

Figure 9. Measurement of the scintillation efficiency of small 1-inch by 1-cm thick samples of scintillator materials exposed to $1 \mathrm{Mrad}$ of ${ }^{60} \mathrm{Co}$ irradiation. What is plotted is the ratio of light yield after irradiation to before irradiation for samples as a function of their maximum emission wavelength. The results indicate that for these very small samples, recovery to $90 \%$ of original efficiency occurs for essentially all materials. As such these measurements are not particularly informative. More definitive studies are required of actual detector materials (tiles and fibers in needed lengths) in order to draw meaningful conclusions. 


\section{Conclusions}

From the studies carried out in this program, a wide variety of new scintillation and waveshifter materials has been produced and studied with fluorescence emission wavelengths in the middle of the visible spectrum and with rapid fluorescence decay times and reasonable efficiency. Of these, several promising materials have been produced, and a number of these have been incorporated into multi-clad fiber and tested as waveshifters in tile-fiber geometry. Initial measurements indicate that at least two of these, DSB1 and DSB2, offer superior performance in fluorescence decay time to conventional Y11 waveshifter while maintaining high fluorescence efficiency. These materials are therefore potential replacements for Y11 in any application where fast timing is required, or where improved signal to noise would be an advantage by utilizing short integration times.

\section{Acknowledgments}

We would like to thank the staff of the Notre Dame Radiation Laboratory for the use of the nitrogen laser spectrometer, A. Pla-Dalmau of Fermilab for assistance with the sample irradiation studies, E. Skup of Fermilab for tile grooving, and K. Kephart of Fermilab for tile polishing and preparation, and to the University of Notre Dame for support for facilities and resources for high school students and teachers at the Notre Dame QuarkNet Center.

\section{References}

1. SCIFI97 Conference on Scintillating and Fiber Detectors, A.D. Bross, R .C. Ruchti, and M.R. Wayne, Eds, AIP Conference Proceedings 450 (1998).

2. H. Leutz, Nucl. Instr. Method A 369:422 (1995).

3. R. C. Ruchti, The Use of Scintillating Fibers for Charged-Particle Tracking, Annu. Rev. Nucl. Part. Sci. 1996. 46: 281-319.

4. The CDF II Technical Design Report, Fermilab-Pub-96/390-E.

5. CMS The Hadron Calorimeter Project Technical Design Report, CERN/LHCC 97-31.

6. ATLAS Tile Calorimeter Technical Design Report (TDR), CERN/LHC/9642.

7. The dye Y11 is variously called K27. 


\title{
FAST, LONG-WAVELENGTH SCINTILLATORS AND WAVESHIFTERS ${ }^{*}$
}

\author{
K. ANDERT, D. ASHIKA, B. BAUMBAUGH, A. BROTHERS, H. GUNTHER, \\ J. GURROLA, D. KARMGARD, T. MADLEM, J. MARCHANT, P. MCGOUGH, \\ M. MCKENNA, R. RUCHTI ${ }^{\dagger}$, J. THOMPSON AND M. VIGNEAULT \\ Department of Physics and QuarkNet Center, University of Notre Dame \\ Notre Dame, Indiana 46556, USA
}

L. HERNANDEZ AND C. HURLBUT

Ludlum Measurements Inc, 501 Oak Street Sweetwater, Texas 79556, USA

\begin{abstract}
Studies are presented of new blue-green to red emitting scintillator and waveshifter materials for tracking and calorimetry applications for the detection of ionizing radiation. Materials include plastic scintillators, liquid scintillators, and plastic scintillating and waveshifting fibers. Program goals are to develop faster and more efficient detection media for a variety of experimental applications.
\end{abstract}

\section{Introduction}

This paper focuses on the development of scintillating and waveshifting materials for calorimetry and tracking applications. Ultimate objectives are to identify and produce materials with high quantum efficiency, fast fluorescence decay and that afford tolerance to radiation exposure.

For calorimetric detectors, a common detector technique is the scintillation sampling calorimeter. ${ }^{1,2,3}$ Plates or "tiles" of blue-violet emitting scintillator are read out with embedded multi-clad waveshifting fiber containing the dye Y11. Light emission is near $\lambda=510 \mathrm{~nm}$ and the fluorescence decay time is relatively long $>7 \mathrm{~ns}$. This paper provides preliminary measurements of scintillating organic plastic and liquid materials containing new fluorescent dyes that are potential replacements for conventional scintillator and waveshifter. In a high radiation environment, scintillator-based liquids may be flushed from a system

\footnotetext{
* This work is supported in part by the U. S. Department of Energy SBIR and STTR Programs, the U. S. National Science Foundation and Department of Energy QuarkNet Program, the National Science Foundation RET Program and the University of Notre Dame.

${ }^{\dagger}$ Current address is the National Science Foundation, Arlington, VA 22230 USA.
} 
and replaced by new material as needed. Additionally several of these dyes have been incorporated as waveshifters in multiclad fiber for the readout of tile scintillators.

\section{Scintillators and Wavelength Shifters}

Bulk scintillator and waveshifter samples were polymerized in either polystyrene or polyvinyltoluene. Liquid samples were prepared from aromatic, organic liquid-scintillator base. Fiber scintillator and waveshifters were produced with double-cladding.

\subsection{Optical Characteristics of Scintillators and Waveshifters}

Initially the materials were characterized optically. Emission and excitation spectra for each of the samples were measured with a Varian Cary Eclipse Fluorescence Spectrophotometer attached to a custom data acquisition system. Figure 1 a sample of emission spectra for three new shifter dyes, DSF1 and AH238B in organic liquid base and DSF2 in a polyvinyltoluene base.

The fluorescence decay times of the samples were measured with a Horiba Jobin Yvon Pulsed Diode Laser Excitation System with TBX-04 Picosecond Photo Detection Module. Samples were excited at $\lambda=373 \mathrm{~nm}$ and the fluorescence decay observed at emission maximum. As can be seen in Figure 2, the photoexcited fluorescence decay times of DSF1 ( $\tau=1.3 \mathrm{~ns})$ is significantly faster than standard Y11 $(\tau=7.4 \mathrm{~ns})$ by a factor of over 5 . The decay of AH238B is slower ( $\tau=11.5 \mathrm{~ns}$ ), but the emission wavelength for this dye is considerably longer (in the yellow/red). 


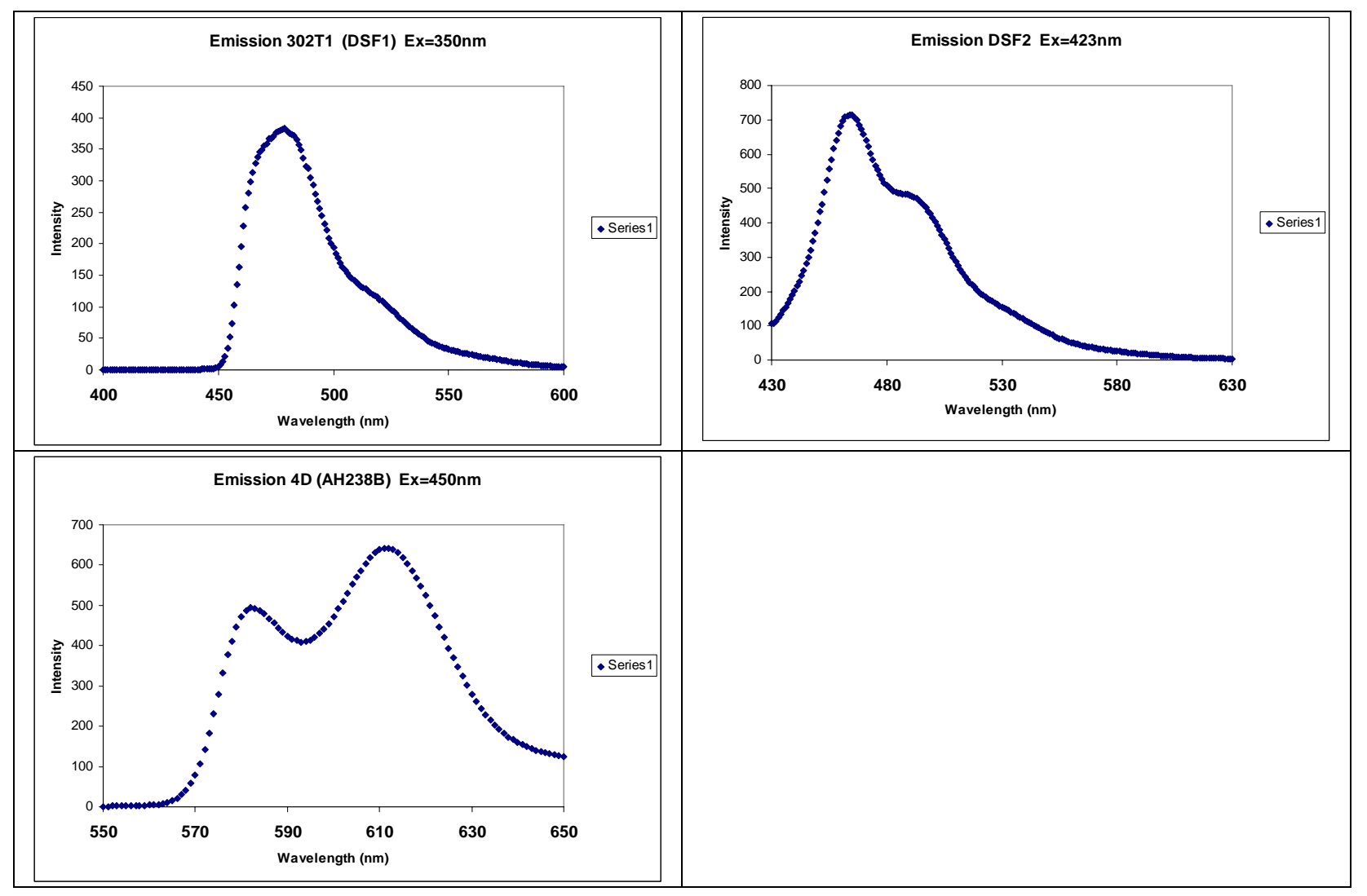

Figure 1. Emission spectra for several waveshifters. DSF1 waveshifter organic liquid base (upper left). DSF2 waveshifter in PVT base (upper right), AH238B waveshifter in organic liquid base (lower left). 


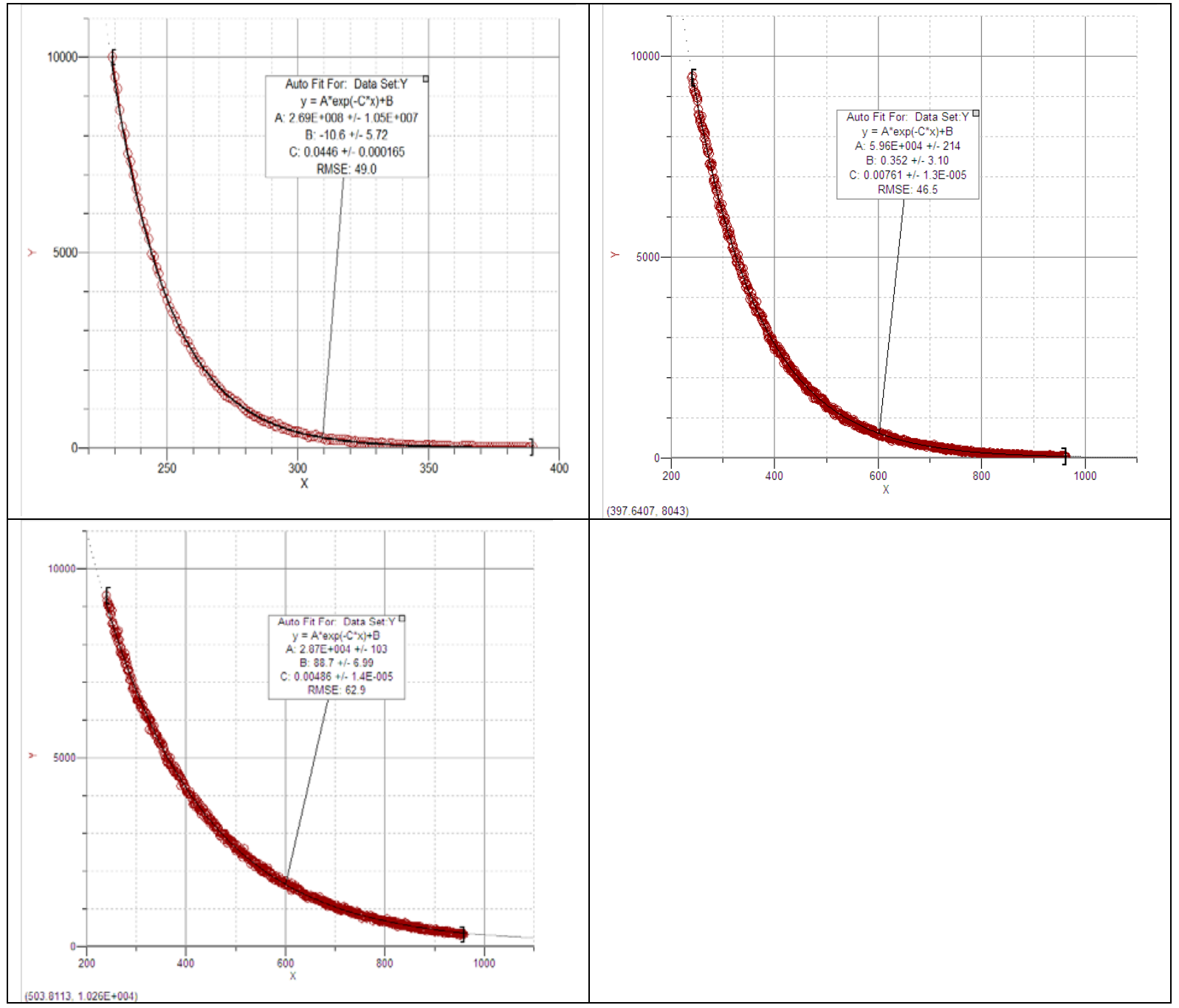

Figure 2. Fluorescence decay times excited by a Diode Laser at $377 \mathrm{~nm}$. Light is detected at the emission maximum for the samples. Fits to the curves indicate the following: DSF1 in organic liquid (upper left, 1.3ns); Y11 in PVT (upper right, 7.4ns); AH238B in organic liquid (lower left, $11.5 \mathrm{~ns})$. 


\subsection{Scintillation Efficiency Studies}

Efficiencies of the scintillator samples were characterized by exposing them to a ${ }^{90} \mathrm{Sr}$ beta source. Signals were detected with a Hamamatsu R1104 photo multiplier connected to a LeCroy QVT pulse height analyzer and read into a custom computer data acquisition system. Optical filtering was used between the samples and the phototube to cut out emission from the bulk material and other primary dyes (if used). ${ }^{4}$ Table 1 displays the measured scintillation efficiencies as a function of wavelength for several scintillation materials prepared for this study. The efficiencies are corrected (scaled) for the wavelength dependent quantum efficiency of the R1104 PMT.

Table 1. Scintillation Efficiency of Samples

\begin{tabular}{|c|c|c|c|c|}
\hline \multirow[t]{3}{*}{ Composition } & Primary & Secondary & Emission & Efficiency \\
\hline & Dye & Dye & Wavelength & Relative \\
\hline & & & $(\mathrm{nm})$ & To BBQ \\
\hline Liquid L1 & $\mathrm{AH} 238 \mathrm{~B}$ & & 600 & 0.84 \\
\hline Liquid L1 & P1 & & 450 & 1.05 \\
\hline Liquid L1 & P1 & $\mathrm{AH} 238 \mathrm{~B}$ & 600 & 1.42 \\
\hline Liquid L1 & $\mathrm{P} 2$ & BBQ & 470 & 1 \\
\hline Liquid L1 & P3 & DSF1 & 480 & 1.02 \\
\hline Liquid L1 & P3 & DSB1 & 500 & 1.11 \\
\hline Liquid L1 & P3 & DSB2 & 510 & 1.25 \\
\hline Liquid L2 & P3 & DSF1 & 480 & 1.08 \\
\hline Liquid L2 & P3 & DSF2 & 480 & 1.02 \\
\hline
\end{tabular}




\section{Tile-Fiber Measurements}

Those secondary dyes that revealed promising scintillation performance and rapid fluorescence decay were then incorporated into double-clad fiber for waveshifter studies. The dye materials so chosen included DSF1, DSF2, AH238B and were compared to the standard dye Y11. These were used to read out scintillation tiles of $10 \mathrm{~cm} \times 10 \mathrm{~cm}$ dimension and $6 \mathrm{~mm}$ thickness of scintillation material (EJ200) with emission maximum in the blue-violet $(\lambda=$ $425 \mathrm{~nm})$. The detection system has been described elsewhere ${ }^{4}$ and includes a three-fold coincidence of trigger counters, including a PMT that views the scintillation tile itself and two other thin independent counters one above and one below the tile. The coincidence of these counters is used as a gate for the signal from the waveshifter fiber, located in a groove in the tile. The optical signal from the fiber is read out using a Hamamatsu H7422P-40 GaAsP PMT. The signals from the PMT were averaged over 2048 pulses and displayed and recorded on an HP54502A digitizing oscilloscope and read into a computer. Such signals were recorded for cosmic ray triggers and for triggers from a ${ }^{90} \mathrm{Sr}$ beta source.

Figure 3 displays the recorded signals from the tile/fiber combinations. As can be seen, the best performance is observed for DSB1 shifter and it is superior to Y11 in both overall signal as well as signal response time. DSF1 and DSF2 have lower overall signal, but have very fast response. The data show that for DSF1, the full width at half maximum (FWHM) is 9.0ns and a fit to the tail with a single exponential function reveals a decay time of $\tau=6.1 \mathrm{~ns}$. The values of these quantities for Y11 are FWHM $=17 \mathrm{~ns}$ and $\tau=12.1 \mathrm{~ns}$, indicating the superiority of the DSF1 in time response. Table 2 summarizes the results of these measurements and others. 

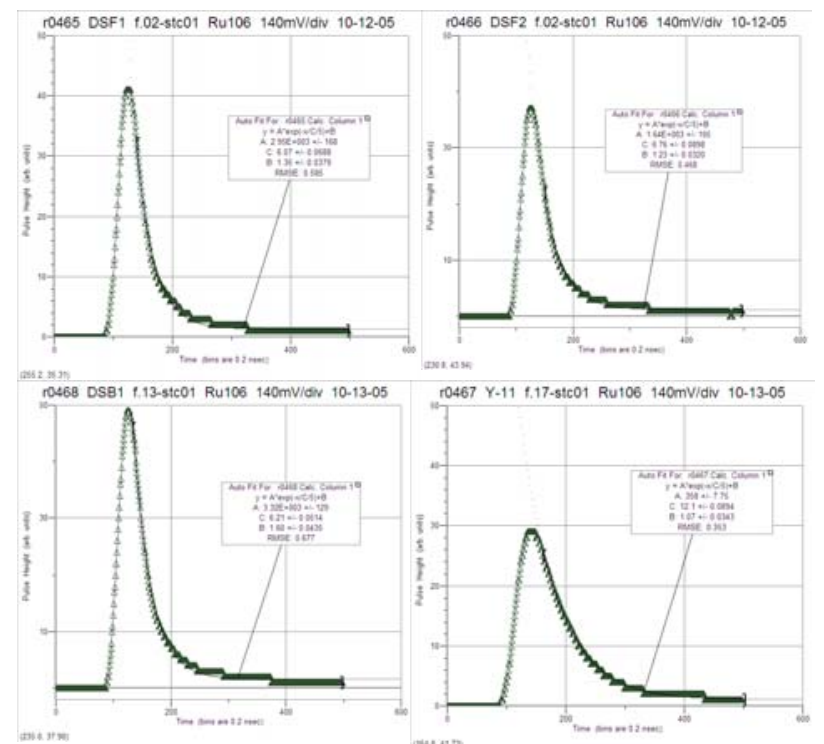

Figure 3. Averaged output pulses from various waveshifters in double-clad fiber of $940 \mathrm{~mm}$ diameter and $1 \mathrm{~m}$ length reading out EJ200 scintillating tile of $6 \mathrm{~mm}$ thickness and $10 \mathrm{~cm} \times 10 \mathrm{~cm}$ area. Photosensor is a GaAsP PMT. DSF1 (upper left); DSF2 (upper right); DSB1 (lower left); Y11 in PVT (upper right). Values are summarized in Table 2 below.

Table 2. Summary of Waveshifting Efficiency for Sample Materials.

$\begin{array}{llllll}\text { Waveshifter } & \begin{array}{l}\text { Emission } \\ \text { wavelength }\end{array} & \begin{array}{l}\text { Efficiency rel. } \\ \text { to Y11 }\end{array} & \begin{array}{l}\text { Decay Time } \\ \text { Source Exc. }\end{array} & \begin{array}{l}\text { FWHM } \\ \text { Source Exc. }\end{array} & \begin{array}{l}\text { Decay Time } \\ \text { Laser Exc. }\end{array} \\ \text { Y11 } & 529 \mathrm{~nm} & 1 & 12.1 \mathrm{~ns} & 17 \mathrm{~ns} & 7.4 \mathrm{~ns} \\ \text { DSB1 } & 500 \mathrm{~nm} & 1.02 & 6.2 \mathrm{~ns} & 9.4 \mathrm{~ns} & \\ \text { DSF1 } & 480 \mathrm{~nm} & 0.84 & 6.1 \mathrm{~ns} & 9.0 \mathrm{~ns} & 1.3 \mathrm{~ns} \\ \text { DSF2 } & 480 \mathrm{~nm} & 0.79 & 6.6 \mathrm{~ns} & 9.6 \mathrm{~ns} & \end{array}$




\section{Conclusions}

From the studies underway in this program, several new scintillation and waveshifter materials have been produced and studied with fluorescence emission wavelengths in the red to green and with rapid fluorescence decay times and reasonable efficiency. These have been incorporated into multi-clad fiber and tested as waveshifters in tile-fiber geometry (DSF1, DSF2, DSB1, DSB2). Additionally, several of these have been used as secondaries in liquid scintillation cocktails (DSF1, DSF2, DSB1, DSB2, and AH238B). Initial measurements indicate that DSB1, DSF1 and DSF2 offer superior performance in fluorescence decay time to conventional Y11 waveshifter. DSB1 and DSF1 offer comparable integral fluorescence efficiency. These materials are therefore potential replacements for Y11 in any application where fast timing is required, or where improved signal to noise would be an advantage by utilizing short integration times.

\section{Acknowledgments}

We would like to thank P. Kamat and the staff of the Notre Dame Radiation Laboratory for the use of the Diode laser spectrometer, E. Hahn of Fermilab for fiber end-polishing, and to the University of Notre Dame for support facilities and resources for high school students and teachers at the Notre Dame QuarkNet Center.

\section{References}

1. The CDF II Technical Design Report, Fermilab-Pub-96/390-E.

2. CMS The Hadron Calorimeter Project Technical Design Report, CERN/LHCC 97-31.

3. ATLAS Tile Calorimeter Technical Design Report (TDR), CERN/LHC/9642.

4. M. Albrecht, et al, Scintillators and Wavelength Shifters for the Detection of Ionizing Radiation, Astroparticle, Particle and Space Physics, Detectors ad Medical Physics Applications, ICATPP-8, M. Barone, E. Borchi, C. Leroy, P.-G. Rancoita, P.-L. Riboni, R. Ruchti, eds, World Scientific, 502511 (2004). 


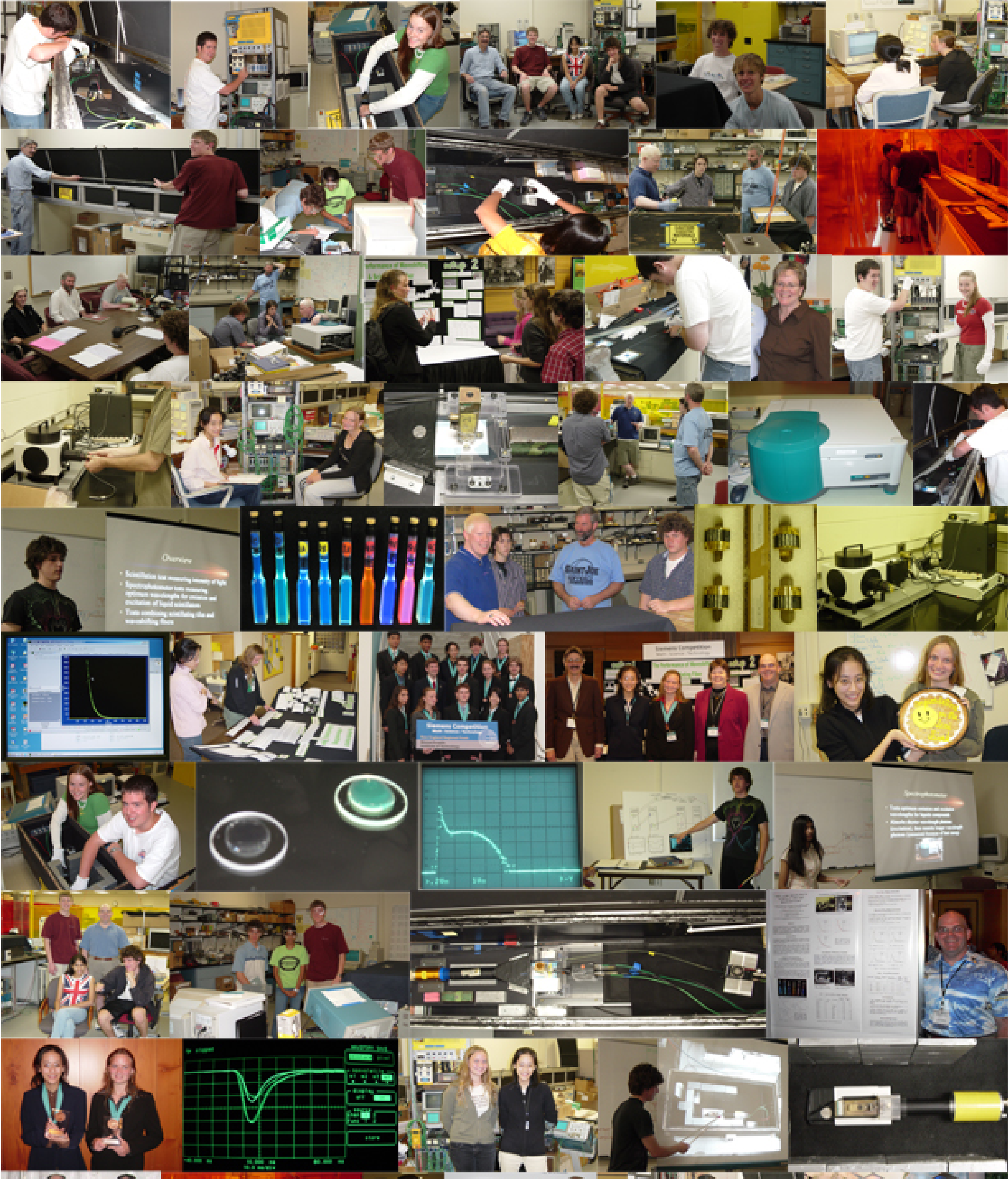

